\title{
Folded Compact Range Development and Coherent Change Detection Measurement Project
}

\section{Kurt W. Sorensen}

\section{Prepared by}

Sandia National Laboratories

Albuquerque, New Mexico 87185 and Livermore, California 94550

for the United States Department of Energy

under Contract DE-AC04-94AL85000

Approved for public release; distribution is unlimited.

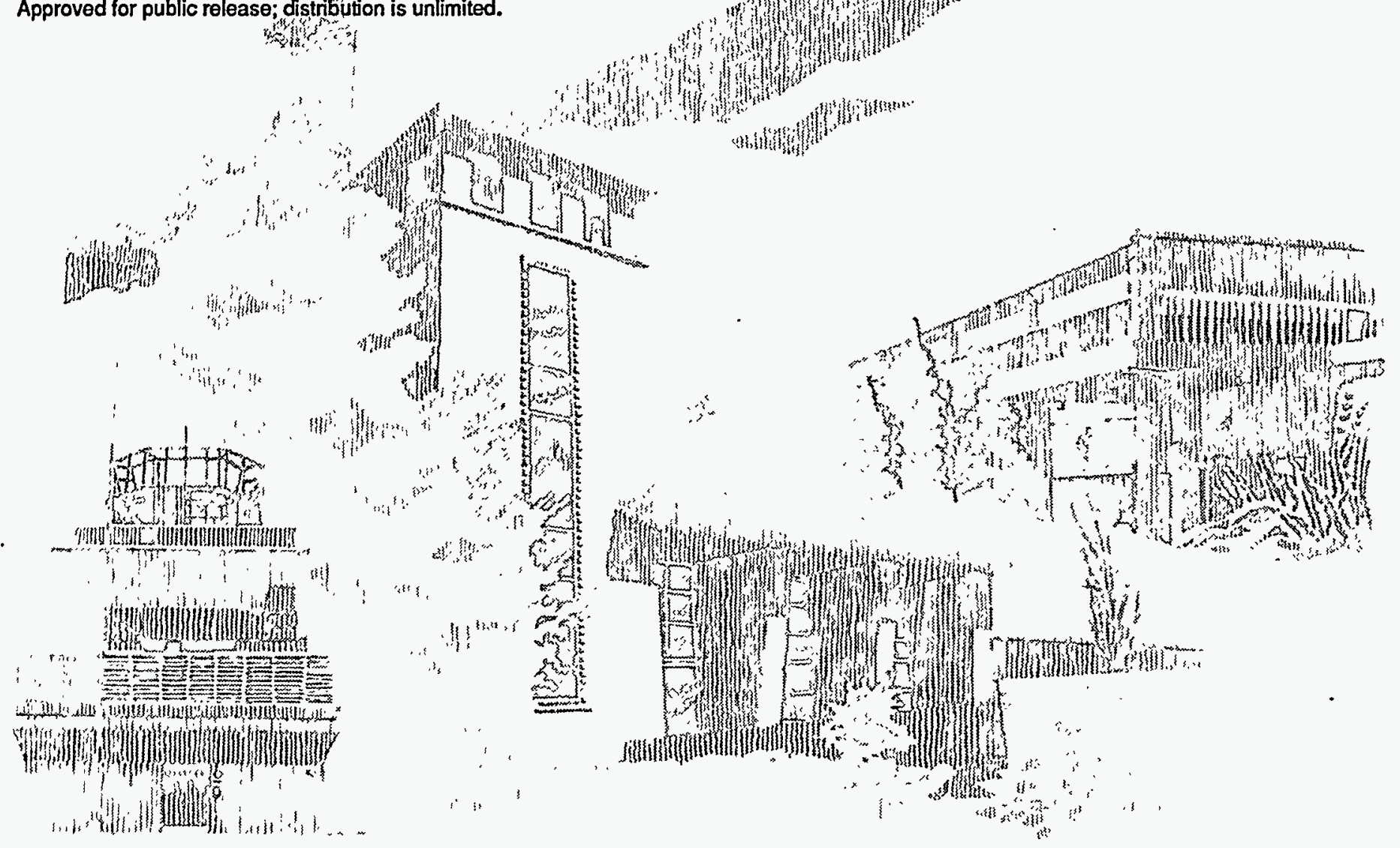


Issued by Sandia National Laboratories, operated for the United States Department of Energy by Sandia Corporation.

NOTICE: This report was prepared as an account of work sponsored by an agency of the United States Government. Neither the United States Government nor any agency thereof, nor any of their employees, nor any of their contractors, subcontractors, or their employees, makes any warranty, express or implied, or assumes any legal liability or responsibility for the accuracy, completeness, or usefulness of any information, apparatus, product, or process disclosed, or represents that its use would not infringe privately owned rights. Reference herein to any specific commercial product, process, or service by trade name, trademark, manufacturer, or otherwise, does not necessarily constitute or imply its endorsement, recommendation, or favoring by the United States Government, any agency thereof or any of their contractors or subcontractors. The views and opinions expressed herein do not necessarily state or reflect those of the United States Government, any agency thereof or any of their contractors.

Printed in the United States of America. This report has been reproduced directly from the best available copy.

Available to DOE and DOE contractors from

Office of Scientific and Technical Information

PO Box 62

Oak Ridge, TN 37831

Prices available from (615) 576-8401, FTS 626-8401

Available to the public from

National Technical Information Service

US Department of Commerce

5285 Port Royal Rd

Springfield, VA 22161

NTIS price codes

Printed copy: A05

Microfiche copy: A01 


\section{DISCLAIMER}

Portions of this document may be illegible in electronic image products. Images are produced from the best available original document. 
SAND95-0389

Distribution

Unlimited Release

Category UC-706

Printed March 1995

\title{
FOLDED COMPACT RANGE DEVELOPMENT \\ AND \\ COHERENT CHANGE DETECTION \\ MEASUREMENT PROJECT
}

\author{
Kurt W. Sorensen \\ Radar/Antenna Department \\ Sandia National Laboratories \\ Albuquerque, NM 87185-0533
}

\begin{abstract}
A novel, folded compact range configuration has been developed at the Sandia National Laboratories' compact range antenna and radar cross section measurement facility, operated by the Radar/Antenna Department 2343, as a means of performing indoor, environmentallycontrolled, far-field simulations of synthetic aperture radar (SAR) coherent change detection (CCD) measurements. This report describes the development of the folded compact range configuration, as well as the initial set of coherent change detection measurements made with the system. These measurements have been highly successful, and have demonstrated the viability of the folded compact range concept in simulating SAR CCD measurements. It is felt that follow-on measurements have the potential of contributing significantly to the body of knowledge available to the scientific community involved in CCD image generation and processing, and that this tool will be a significant aid in the research and development of change detection methodologies.
\end{abstract}

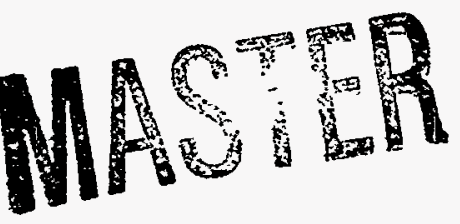


Intentionally Left Blank 


\section{CONTENTS}

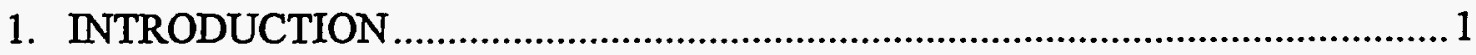

2. COMPACT RANGE RECONFIGURATION ……......................................... 1

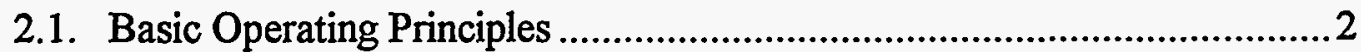

2.2. Near Field Considerations ..........................................................................

2.3. Secondary Planar Reflector.....................................................................5

2.4. Folded Compact Range Configuration ..................................................... 6

2.5. Field Probe Measurements .................................................................. 8

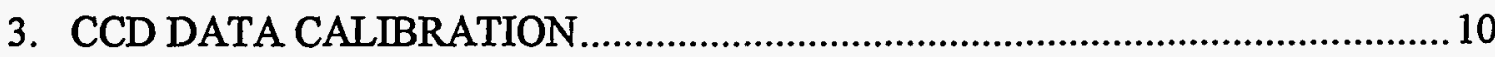

3.1. Radar Parameters .............................................................................. 10

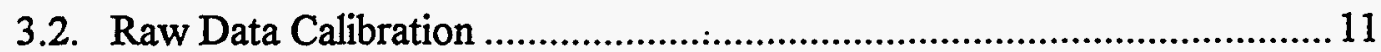

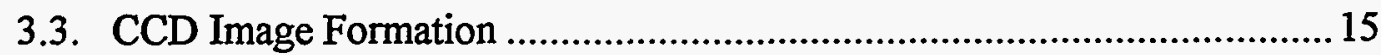

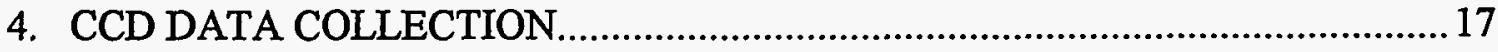

4.1. CCD Image Resolution.......................................................................... 17

4.2. Test Plan for CCD Measurements...................................................... 18

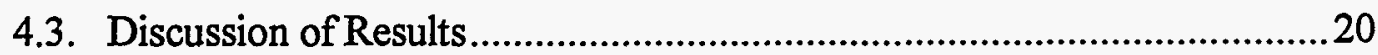

5. PROPOSAL FOR FOLLOW-ON CCD MEASUREMENTS ….............................22

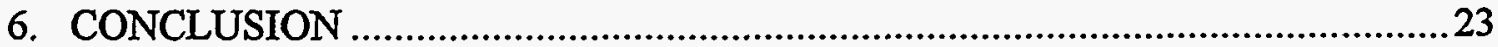

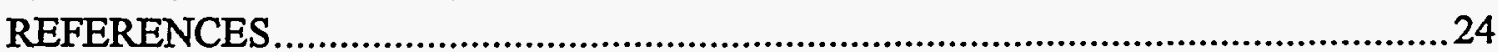

APPENDIX A--CCD Images Processed Using Coherent Subtraction............................ A-1

APPENDIX B--CCD Images Processed Using Maximum Likelihood Estimate of Correlation Algorithm........................................................................ B-1

\section{Figures}

1. Fundamental layout of prime-focus fed compact range measurement system............ 1

2. Measured RCS vs. aspect angle of a 13 in. $x 13$ in. rectangular flat plate at a

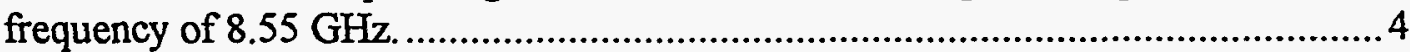

3. Measured near-field amplitude of a 13 in. $x 13$ in. rectangular flat plate at 8.55 $\mathrm{GHz}$.

4. Solar concentrator stretched-membrane facet mounted as secondary planar reflector in SNL compact range measurement facility. .............................................. 6

5. SNL compact range coherent change detection measurement setup........................ 7

6. Coordinates used in computation of bistatic scattering from flat, circular disk.........8

7. Calculated bistatic scattering from 124 in. diameter, perfectly conducting circular disk at $12 \mathrm{GHz}$ with uniform plane wave incident at an angle of 18 degrees............. 8

8. Measured relative magnitude and phase of vertically-polarized electromagnetic field incident upon the sample area at $12 \mathrm{GHz}$....................................................9

9. Photographs of compact range configuration used to perform coherent change

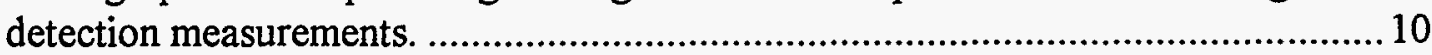

10. Reference target measurement setup used in calibration of raw data. Calibration standard used was a triangular trihedral corner reflector. 
11. Two-dimensional image of four spheres having diameters of $3 / 16,1 / 4,5 / 16$, and $3 / 8$ in., illustrating the resolution capability and dynamic range of the SNL compact range $\mathrm{CCD}$ measurement system.

12. Two-dimensional image of plastic model airplane and corner reflector trihedral mounted on absorber on top of target sample holder. 


\section{INTRODUCTION}

A novel, folded compact range configuration has been developed at the Sandia National Laboratories' compact range antenna and radar cross section measurement facility, operated by the Radar/Antenna Department 2343, as a means of performing indoor, environmentallycontrolled, far-field simulations of synthetic aperture radar (SAR) coherent change detection (CCD) measurements. The folded compact range has been realized by introducing a secondary planar reflector into the compact range in order to direct collimated electromagnetic energy onto distributed targets (soil samples, gravel, etc.) which are placed in a large ( 8 foot diameter) specimen holder at ground level. The compact range employs a gated-CW radar which provides the capability to perform wideband ( $2-18 \mathrm{GHz}$, continuous), fully-polarimetric, coherent measurements, resulting in the generation of high-resolution, twodimensional images having pixel sizes on the order of $1.0 \mathrm{in}^{2}$ or less.

Initial measurements made in the compact range on gravel samples have been highly successful, and have demonstrated the viability of the folded compact range concept in simulating SAR CCD measurements. It is therefore felt that follow-on measurements have the potential of contributing significantly to the body of knowledge available to the scientific community involved in CCD image generation and processing, and that this tool will be a significant aid in the research and development of change detection methodologies.

The purpose of this report is to describe the compact range configuration that was developed to support this research task, as well as the results of the initial set of measurements made with the system.

\section{COMPACT RANGE RECONFIGURATION}

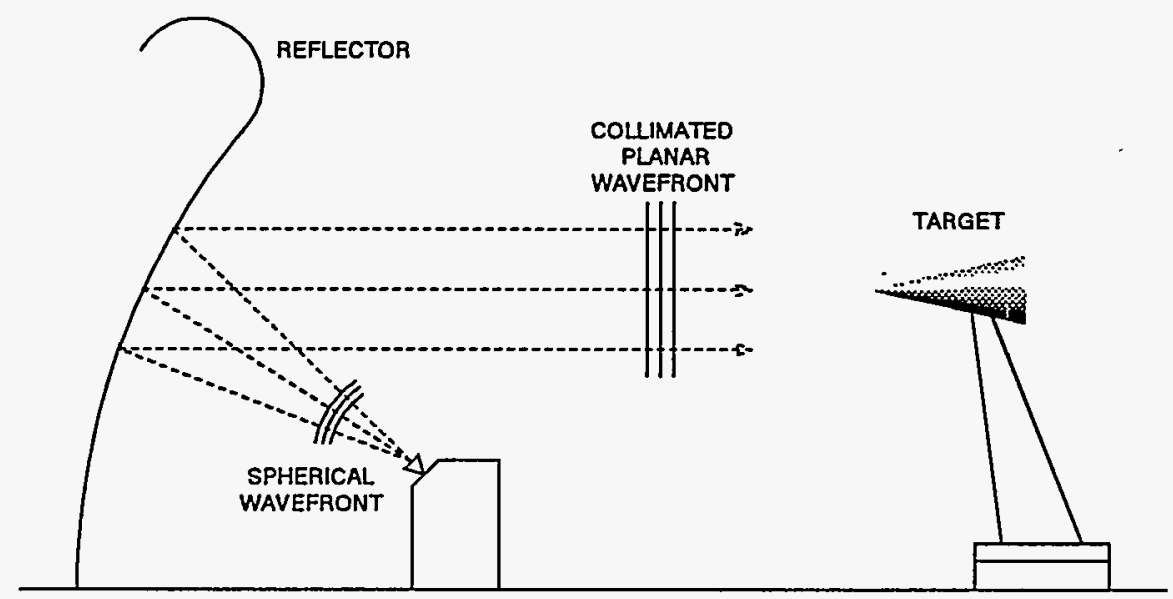

Figure 1. Fundamental layout of prime-focus fed compact range measurement system.

The SNL compact range antenna and RCS measurement facility is fundamentally configured as a prime-focus fed, single parabolic reflector system, as depicted in Figure 1. This system 
can be operated in an inverse synthetic aperture radar (ISAR) mode, in which objects are simultaneously rotated and irradiated with a uniform plane wave over a relatively wide bandwidth of frequencies, following which signal processing is used to form two-dimensional radar images of the illuminated target. It is well known that data identical to that acquired with a circular spotlight SAR can be obtained by equivalent ISAR processing [1]. It was therefore conjectured that this system could also be used to perform indoor, environmentallycontrolled, far-field simulations of SAR CCD measurements. The intent of this section is to discuss how this was accomplished.

\subsection{Basic Operating Principles}

As seen in Figure 1, the SNL compact range basically consists of (in addition to the associated RF hardware--signal generators, amplifiers, etc.) an anechoic chamber, a large rolled-edge parabolic reflector, an antenna feed located at the focus of the parabolic reflector, and a turntable and target mount upon which objects are mounted for testing. The feed antenna simulates a point source of radiation which illuminates the reflector with a spherical wave of electromagnetic energy. The diverging rays from the feed are collimated by the reflector to form a uniform plane wave at the aperture of the reflector; this electromagnetic (EM) field of energy continues to propagate along the axis of the reflector as a good approximation of a plane wave for a distance of several reflector radii [2].

The volume of space in the anechoic chamber in which the EM field can be considered to be both uniform (constant amplitude) and planar (constant phase) is constrained by several factors, including the illumination pattern of the feed antenna, the amount of energy diffracted from the edges of the parabolic reflector, and the amount of other stray radiation existent in the chamber. The purpose of the rolled edges on the parabolic reflector, for example, is an attempt to limit the amount of edge diffraction from the reflector into the target area by creating a geometrical transition to the paraboloidal surface of the reflector which re-directs the radiated energy from the edges into areas immediately adjacent to or behind the reflector, rather than into the target area. The target area or "quiet zone" of the SNL compact range is specified to be $2 \mathrm{~m}$ in width and height. At X-Band, the field in the quiet zone area has been specified to have the following properties [3]:

$$
\begin{aligned}
& \text { Phase ripple: } \quad \pm 10^{\circ} \max \\
& \text { Amplitude ripple: } \pm 0.25 \mathrm{~dB} \max \\
& \text { Amplitude taper: } 0.5 \mathrm{~dB} \max .
\end{aligned}
$$

The compact range quiet zone is centered approximately 13 feet above floor level. This location is rather inconvenient for mounting distributed targets (soil, gravel, etc.) for the purposes of making backscatter measurements; it was therefore postulated that a "folded" compact range could be created by placing a secondary planar reflector into the quiet zone of the compact range to re-direct the collimated EM field from the parabolic reflector onto a sample holder which would be placed at ground level. The radar cross section of a target (in this case, a distribution of randomly-oriented scatterers) is by definition a far-field quantity. The objective with regard to implementing a secondary reflector is therefore to simulate a 
uniform plane wave over a region of space at ground level that is large enough to allow reasonably-sized samples ( $\geq 6$ feet in extent) to be measured. Provided that the secondary reflector satisfies the necessary conditions of surface tolerance and accuracy (surface planarity, smoothness, freedom from edge diffraction, etc.), the planarity of the field should essentially be preserved in the sample area located at ground level, since this sample area would be in the near-field region of scattering from the secondary reflector. Then by reciprocity, the backscattered field from the sample to the planar reflector (and then on to the parabolic reflector) would also be planar in nature, such that a reasonably accurate SAR system could be modeled.

\subsection{Near Field Considerations}

The importance of the sample area being in the near field region of scattering from the secondary reflector can be explained by briefly exploring the relationship between the near and far field, and by using an example of scattering from a rectangular flat plate. The "scattering" of electromagnetic energy from a body is actually the electromagnetic field re-radiated from the surface of the object due to currents which have been induced upon it by an incident electromagnetic field. At any point in space these re-radiated electric and magnetic fields can be expressed in terms of a surface integral of the currents induced on the object [4]. Although this integral cannot be readily evaluated in general, approximations to the solution can be obtained by dividing the area about the object into "near-field" and "far-field" regions. The "boundary" between these two regions is usually taken to be either [5]

$$
r=\frac{D^{2}}{\lambda}
$$

or

$$
r=\frac{2 D^{2}}{\lambda}
$$

where $D$ is the diameter of the smallest sphere which would completely enclose the target, and $\lambda$ is the wavelength of the incident field. In the near-field region, the rays radiating from the surface of the object are not parallel, and consequently the radar reflectivity is not constant versus range. In the far-field region, however, the observation point is separated from the scattering object a distance sufficiently large that the radiated rays may be considered to be parallel to one another, and the radar reflectivity is thus constant versus range.

The variability of the radar reflectivity of an object in the near-field region may be used to advantage, however, when trying to simulate a uniform plane wave over a localized area. For example, Figure 2 shows the measured radar cross section (again, a far-field quantity) of a 13 inch $\times 13$ inch conducting, rectangular flat plate. It can be seen that the scattered field beamwidth is quite narrow, and that a $\sin (\mathrm{x}) / \mathrm{x}$ pattern is generated by the energy which is scattered from the two side edges of the flat plate interacting in a constructive and destructive manner. That is, as the phasing between the diffracted energy from the edges varies in conjunction with the variation in aspect angle of the plate, a pattern of peaks and nulls occurs. 


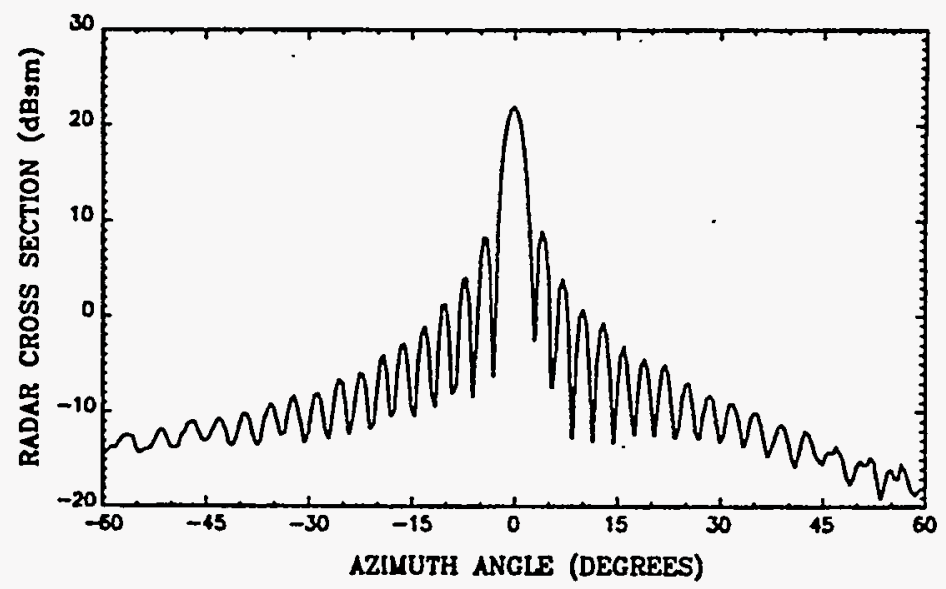

Figure 2. Measured RCS vs. aspect angle of a 13" $\mathrm{x} 13^{\prime \prime}$ rectangular flat plate at a frequency of $8.55 \mathrm{GHz}[6]$.

In contrast, the measured near-field backscatter from the same flat plate exhibits a much more uniform shape, as shown in Figure 3. The probe used to measure the near-field backscatter in this instance was located one inch from the surface of the flat plate. This plot suggests that an object placed at this particular location (one inch from the plate) in the near-field region of the plate would, over a localized area, be illuminated by a field which was essentially uniform and planar in nature.

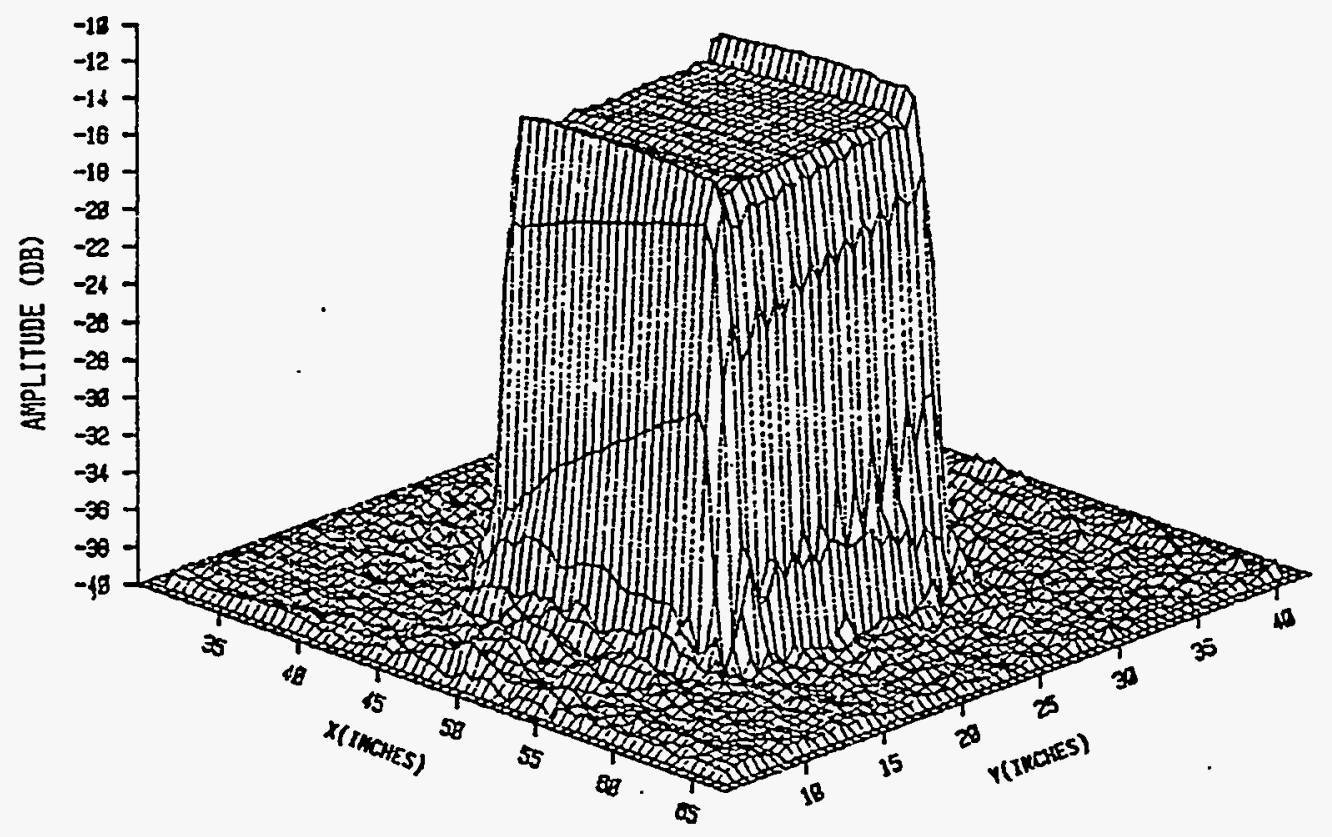

Figure 3. Measured near-field amplitude of a 13" x $13^{\prime \prime}$ rectangular flat plate at $8.55 \mathrm{GHz}$; probe separation distance is 1.0 inch [6]. 


\subsection{Secondary Planar Reflector}

The first order of business in creating a folded compact range, then, was to obtain a "flat plate" large enough to provide for a sample area at ground level roughly equivalent to the nominal quiet zone size of the compact range. This necessitated a planar reflector having dimensions substantially larger than the size of the specified compact range quiet zone $(2 \mathrm{~m} \mathrm{x}$ $2 \mathrm{~m}$ ) in order to insure that the collimated field from the parabolic reflector was adequately intercepted and redirected toward the sample area. Ideally, the reflector would also be large enough that the amplitude of the field incident upon the reflector would be significantly attenuated at the edges (due to the amplitude taper that naturally exists in the quiet zone area of a compact range) in order to decrease the level of extraneous scattering from the edges of the reflector. Furthermore, this planar reflector was required to satisfy stringent requirements for surface tolerance and accuracy. Surface quality criteria for compact range collimating reflectors in general are [7]

$$
\begin{array}{ll}
\text { Surface deviation: } & \leq 0.007 \lambda \\
\text { Surface gaps: } & <\lambda / 20
\end{array}
$$

Applying these same criteria to the desired secondary planar reflector at $12 \mathrm{GHz}$ would require a flat, conductive surface with a tolerance of less than 0.007 " and with gaps no larger than $0.05 "$. It was quickly realized that it would be prohibitively expensive to have such a large flat surface manufactured to such a high degree of accuracy that would also withstand the rigors of being handled and moved about.

In seeking for alternative solutions to this problem, it was discovered that the SNL Solar Thermal Test Facility was nearing the completion of a series of tests on a multi-faceted stretched-membrane dish concentrator. This dish concentrator consisted of twelve $3.15 \mathrm{~m}$ diameter facets that formed a large (approximately $115 \mathrm{~m}^{2}$ ) parabolic dish concentrator which operated a $25 \mathrm{~kW}_{\mathrm{e}}$ Stirling engine for electric power production [8]. Each facet of the dish concentrator is comprised of two 0.003 inch thick stainless steel membranes stretched over each side of a metal ring. The membranes are fabricated by welding flattened stainless steel coil stock strips ( 24 inches wide) together with a roll-resistance seam welder: The entire membrane is subsequently welded to the facet ring. These membranes are tensioned to 20,000 pounds per square inch. The membranes thus have a nominally flat, planar surface at rest. (The vacuum between the two membranes can be used to draw the reflective surfaces into a parabolically-focused shape if desired.) The front membrane is laminated with a silverized polymer film to form an optically-reflective surface. The total weight for each facet is approximately $292 \mathrm{lbs}[8]$.

It was recognized that the facets would be nearly ideal for use as the secondary planar reflector in the compact range. Upon completion of solar testing, the Solar Thermal Test Facility intended to place several of the facets in the field for lifetime environmental testing, but was willing to let one of the facets be used in the compact range. Fixturing was therefore designed and fabricated to accommodate its use. Upon delivery of the facet to the compact range measurement facility, it was discovered that the front membrane (laminated with 
silverized polymer film) had sustained minor hail damage. A decision was thus made to utilize the non-laminated membrane surface as the secondary planar reflector. A photograph of the facet and its associated fixturing is showri in Figure 4.

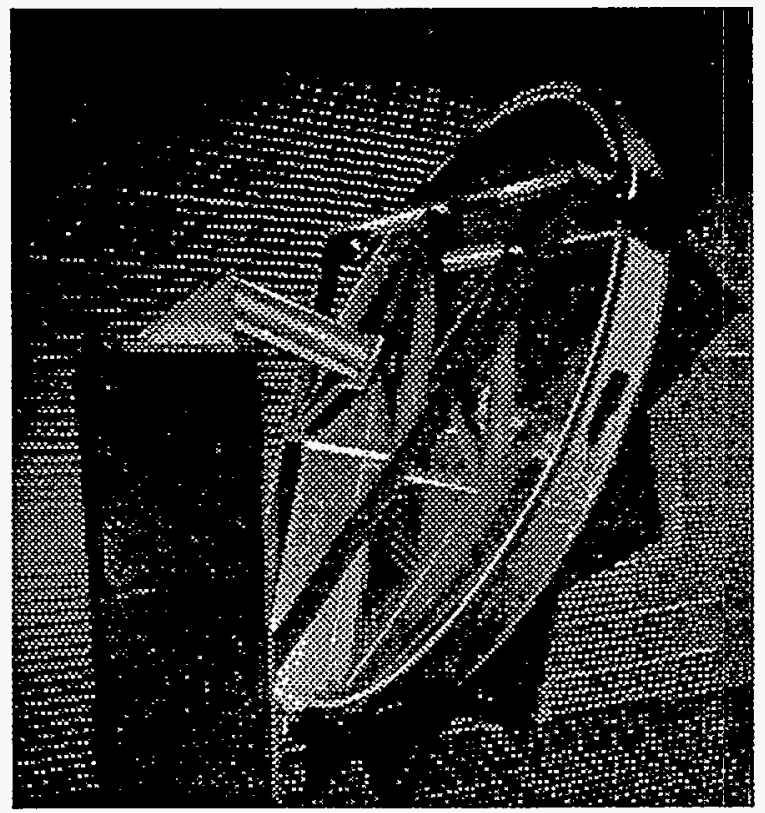

Figure 4. Solar concentrator stretched-membrane facet mounted as secondary planar reflector in SNL compact range measurement facility.

\subsection{Folded Compact Range Configuration}

Snell's law of reflection states that the angle of reflection is equal to the angle of incidence for a uniform plane wave incident on a planar interface at an oblique angle [9]. Simply stated,

$$
\theta_{r}=\theta_{i}
$$

In order to insure that the sample holder was properly illuminated by the incident electromagnetic field, it was therefore necessary to geometrically align the planar solar facet with the existing features of the compact range. This is more conveniently described using the diagram shown in Figure 5. In this diagram it can be seen that, given the height of the compact range quiet zone, the desired location of the sample holder, and the location available for the placement of the planar solar facet, it was necessary to orient the planar reflector at an angle of $18^{\circ}$ relative to vertical. This resulted in a simulated look angle of $36^{\circ}$ for the electromagnetic field incident upon the sample holder, and a distance from the center of the solar facet to the center of the top of the sample holder of approximately 16.7 feet. This is well within the near-field region of scattering from the solar facet, based on the use of Equation (1), from which the distance to the far-field scattering region boundary is calculated to be over 1300 feet. It may also be noted in Figure 4 that microwave absorbing material was applied to the edges of the solar facet in an attempt to create a simulated serrated edge, in 
hopes that diffracted energy from the edges of the facet could be attenuated and/or diverted away from the sample area.

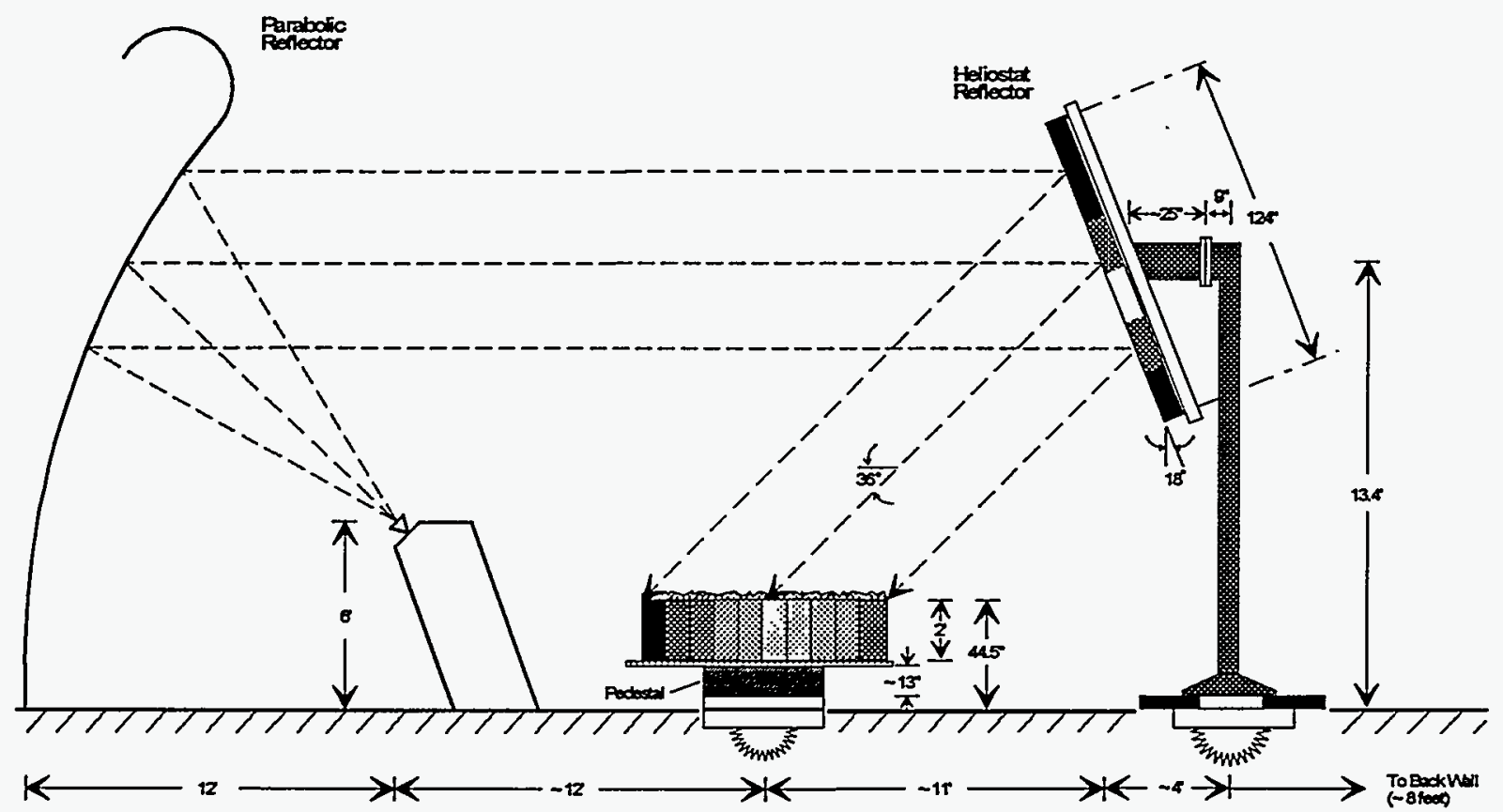

Figure 5. SNL compact range coherent change detection measurement setup.

Far-field bistatic scattering from a perfectly conducting, circular disk has been calculated using Ufimtsev's technique [10], and is shown in Figure 7. The uniform plane wave illuminating the disk in this example is incident at an angle of $18^{\circ}\left(\phi=0^{\circ}\right)$ relative to the surface normal of the disk, as indicated in Figure 6 . As predicted by (3), the peak scattering from the disk is shown in Figure 7 to also occur at an angle of $18^{\circ}\left(\phi=180^{\circ}\right)$.

The principle reason for including this data is to emphasize that, as in the example of far-field scattering from the rectangular flat plate, the energy diffracted from the edges of the disk results in the generation of a $\sin (\mathrm{x}) / \mathrm{x}$ pattern of scattering. This diffracted energy also affects the near-field scattering pattern from the disk, as will be seen below. 


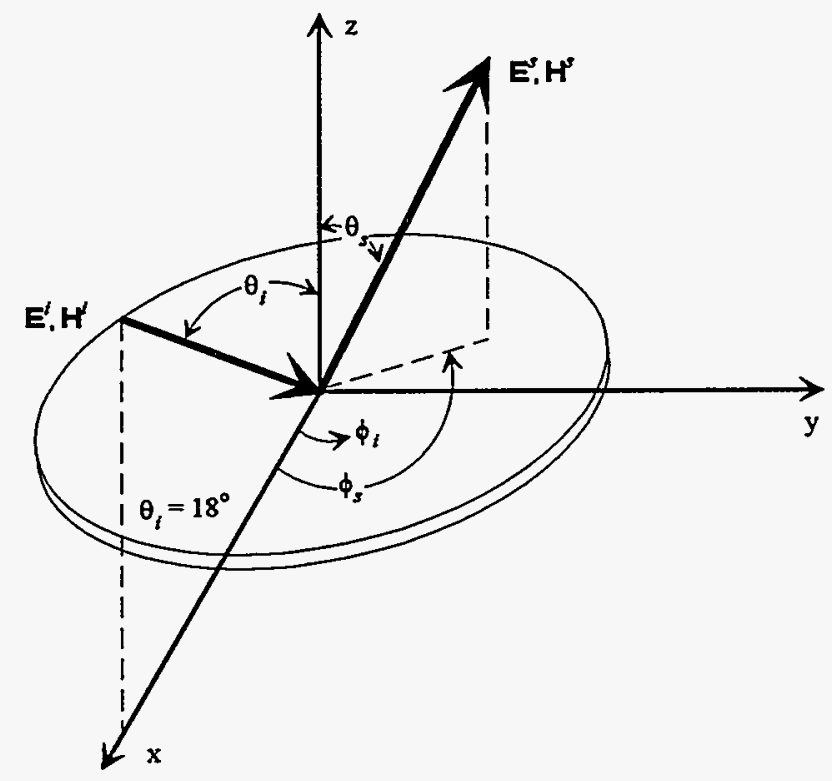

Figure 6. Coordinates used in computation of bistatic scattering from flat, circular disk.

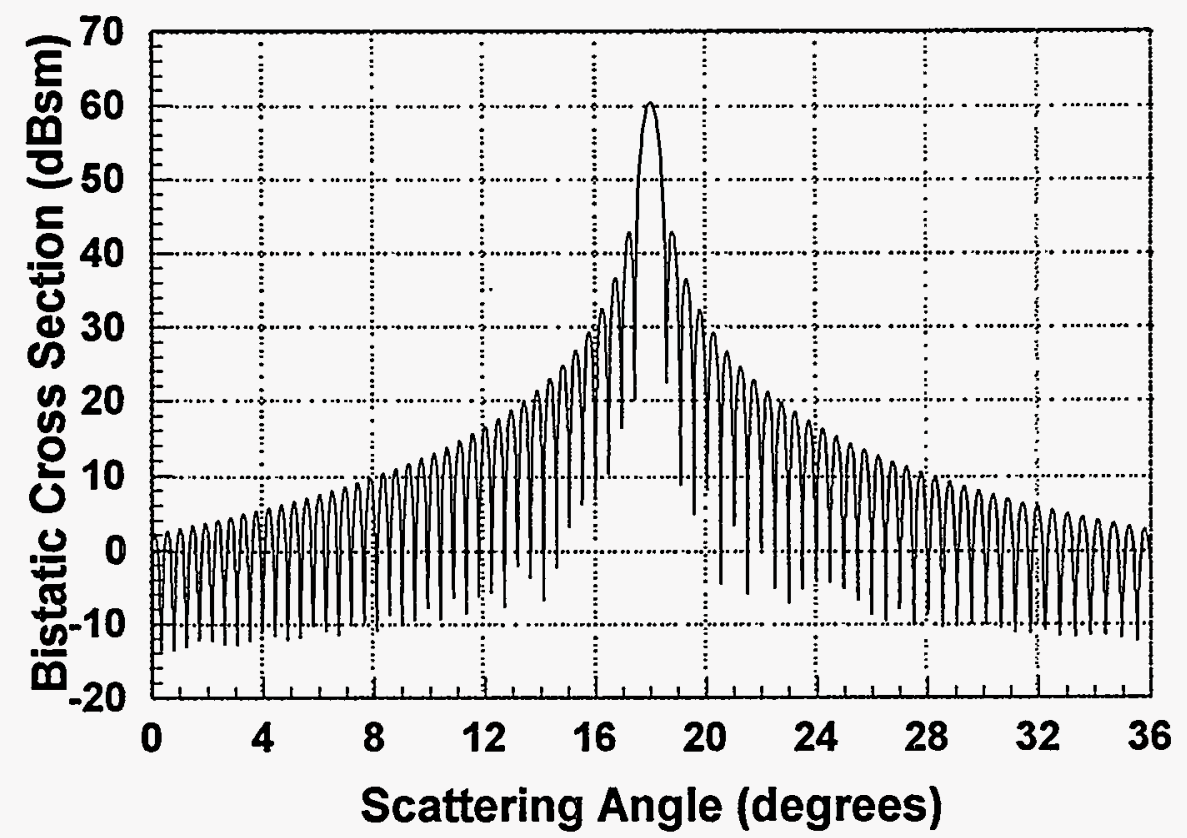

Figure 7. Calculated bistatic scattering from 124" diameter, perfectly conducting circular disk at $12 \mathrm{GHz}$ with uniform plane wave incident at an angle of $18^{\circ}$.

\subsection{Field Probe Measurements}

Prior to placing the sample holder in the compact range, a "field probe" was placed at the position in the compact range at which the sample holder would be located. The field probe measured the amplitude and phase of the electromagnetic field scattered from the solar facet 
at the location of the sample holder so that the field irradiating the sample could be accurately characterized. The field was measured at 8,10 , and $12 \mathrm{GHz}$ for both vertical and horizontal polarizations. Plots of the measured amplitude and phase of the vertically-polarized incident field at $12 \mathrm{GHz}$ are shown in Figure 8. These plots are representative of the data recorded at the other frequencies and polarizations.
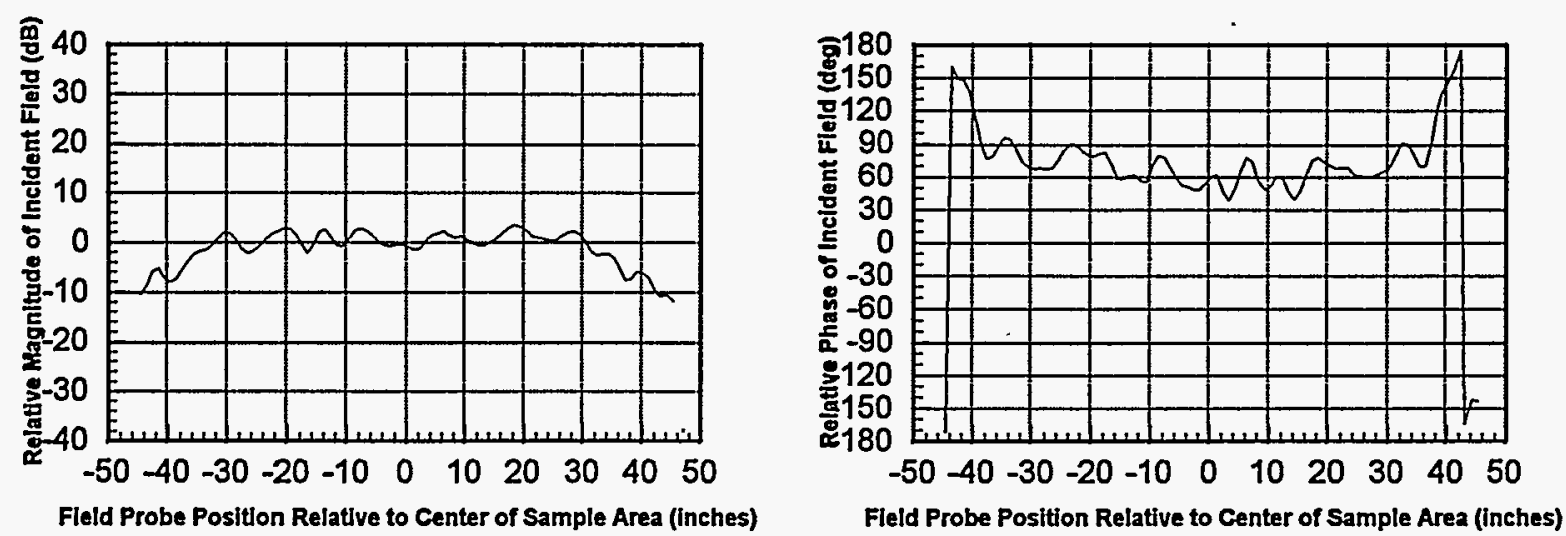

Figure 8. Measured relative magnitude and phase of vertically-polarized electromagnetic field incident upon the sample area at $12 \mathrm{GHz}$. Orientation of the field probe is parallel to the surface of the solar facet ("cross-range cut").

The plots show the field scattered from the solar facet at the desired location of the sample holder to be a reasonable approximation of a uniform plane wave. The amplitude and phase ripple evident in the plots is believed to principally be due to diffracted energy from the edges of the solar facet entering the sample area. From the theoretical far-field analysis of bistatic scattering from a "perfect" circular disk, as shown in Figure 7, it is known that the edges contribute significantly to the backscattering cross section. Further, it is believed that the diameter of the solar facet was not large enough for the amplitude of the incident field at the edges of the facet to be significantly attenuated relative to that over the nominal quiet zone volume; thus, despite the fact that the edges of the facet were treated with absorbing material, it is likely that they are significant contributors of extraneous energy.

From analysis of the measured field-probe data it can be determined that over a 6 foot region in the sample area ( \pm 36 inches about the sample area center) the incident electromagnetic field has the following properties:

Phase ripple: $\quad \pm 30^{\circ}$

Amplitude ripple: $\pm 3 \mathrm{~dB}$

Amplitude taper: $0 \mathrm{~dB}$.

While these values are significantly degraded from the field properties specified for the quiet zone of the compact range, thus precluding use of this range configuration for highly accurate absolute radar-cross-section measurements, it is nevertheless quite adequate (particularly in light of the quality of CCD data gathered with the system) for use in measuring or detecting changes in backscattering levels. 
As determined by the measured quality of the electromagnetic field characteristics in the desired sample area, the size of the "quiet zone" at ground level is thus approximately six feet in diameter. In searching for a sturdy sample holder having a diameter of at least six feet, the most economical and readily-available alternative was a circularly-shaped cattle trough having an 8 foot diameter that could be purchased through a local hardware store. The cattle trough had a depth of 24 inches. In order to attenuate undesired extraneous backscatter from the trough which could perturb the scattered signal from the test target, the trough was lined with microwave absorbing materials on all inner and outer surfaces. The bottom eighteen inches of the trough space were filled with a lightweight, low dielectric constant polystyrene material in order to limit the quantity of sample material which would be necessary to conduct experimental measurements.

Photographs of the final range configuration are shown in Figures 9 and A-1.
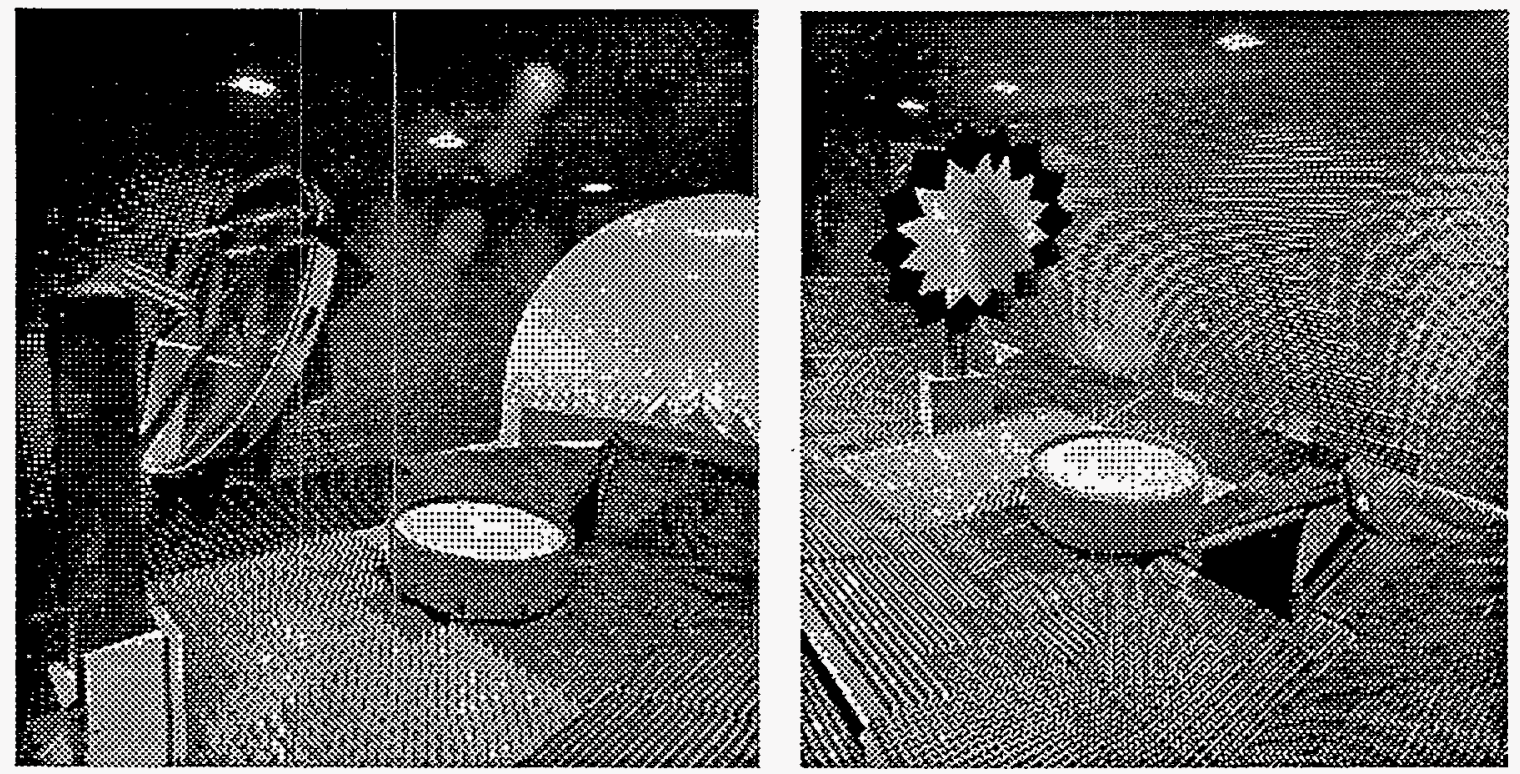

Figure 9. Photographs of compact range configuration used to perform coherent change detection measurements.

\section{CCD DATA CALIBRATION}

\subsection{Radar Parameters}

The SNL compact range measurement system radar is essentially composed of HewlittPackard hardware and Flam and Russell (F\&R) software. The main elements of the radar hardware are an HP8530A microwave receiver and HP83621A frequency synthesizers, along with an assortment of other RF accessories. The radar hardware is wideband, coherent, has high sensitivity and large dynamic range, and is highly stable. In performing radar cross section measurements, the measurement system is operated as a gated-CW radar. That is, clutter (undesired stray EM radiation) in the anechoic chamber is temporally discriminated 
from the desired target return by "turning on" the receiver only during the relatively short window of time at which the backscattered return from the target is expected.

Measurements of the distributed target samples are made by performing wideband swept frequency measurements while rotating the target samples over a prescribed angular sector. A few of the pertinent radar parameters used for the majority of the measurements are given in Table 1.

TABLE 1. Radar Parameters

\begin{tabular}{|l|c|}
\hline $\begin{array}{l}\text { Plane Wave Incidence } \\
\text { Angle }\end{array}$ & $36^{\circ}$ \\
\hline Effective Radar Pulse Width & $25 \mathrm{~ns}$ \\
\hline Frequency Bandwidth & $8.0-16.0 \mathrm{GHz}$ \\
\hline $\begin{array}{l}\text { Number of Frequencies per } \\
\text { Sweep }\end{array}$ & 201 \\
\hline $\begin{array}{l}\text { Number of Integrations per } \\
\text { Frequency Step }\end{array}$ & 32 \\
\hline $\begin{array}{l}\text { Azimuth Rotation Angular } \\
\text { Limits }\end{array}$ & $\pm 17.2^{\circ}$ \\
\hline $\begin{array}{l}\text { Azimuth Rotation Angular } \\
\text { Step Increment }\end{array}$ & $0.15^{\circ}$ \\
\hline Measured Polarizations & Vertical, Horizontal \\
\hline
\end{tabular}

\subsection{Raw Data Calibration}

In order for the data to be useful and quantitative it must be calibrated to eliminate radar- and range-specific dependencies (it should quickly be noted, however, that all raw data collected for each measurement configuration has been saved). While there are various accepted methods for calibrating a radar cross section measurement system, the F\&R software utilizes a "direct" method of calibration [2], in which a calibration standard is used to provide a backscattered radar return that can then be compared with the signal from the test target. The calibration method is described by the following equation [11]:

where

$$
\sigma_{c a l}=\frac{T G T_{\text {test }}(f, \theta, \phi)-B K G_{\text {test }}(f, \theta, \phi)}{T G T_{\text {ref }}(f)-B K G_{\text {ref }}(f)} \times \sigma_{\text {ref }}^{\text {theor }}(f),
$$

$T G T_{\text {test }}=$ uncalibrated measurement of test target data, $B K G_{\text {test }}=$ uncalibrated measurement of target background (clutter) data,

$T G T_{\text {ref }}=$ uncalibrated measurement of reference target, $B K G_{\text {ref }}=$ uncalibrated measurement of reference background clutter, $\sigma_{\text {ref }}^{\text {theor }}=$ theoretical RCS of reference target. 
The "uncalibrated" measured values represented in Equation 4 are complex voltage values which have actually been normalized in the receiver by a transmit reference signal, as well as corrected by IF correction algorithms internal to the receiver [11]. In all other respects, however, the data is in fact uncalibrated.

As shown in (4), the test target data is measured as a function of frequency and two aspect angles (elevation and azimuth). For the measurements described in this report, the elevation axis $(\theta)$ remained constant, so that the data is a function only of frequency and azimuth angle. The test background (clutter) data is normally obtained by measuring the environment in which the target under test will be placed, but without the test target present. This clutter data can then be vectorially subtracted from the actual test target data in an attempt to reduce target independent backscattered contributions from mounting fixtures and other range clutter sources to the measured RCS of the target. This feature was employed when making the initial assessment of the new compact range configuration by measuring a variety of test targets and subtracting the background clutter, but was not used, however, in calibrating the raw data collected in the actual series of measurements made on the distributed target samples (such that $B K G_{\text {test }}$ was set to zero).

Also as seen in (4), the reference target and reference background data were collected only as functions of frequency (that is, at a single angular position). Similar to the $B K G_{\text {test }}$ measurement, the reference background (clutter) data is collected by measuring the environment in which the reference target will be placed, but without the reference target present. As explained above, this clutter data can then be vectorially subtracted from the actual reference target data.

The reference target measurement was made by placing the reference target on top of the distributed target sample as depicted in Figure 10. Measurement of a reference target allows the test target data to be calibrated in units of dBsm. For purposes of image formation, placement of the reference target also determines the center of the image scene.

The calibration standard (reference target) chosen for these measurements was a triangular trihedral corner reflector. In addition to having a physical geometry which allows for accurate calculation of its theoretical RCS (in this case physical optics is used for the calculation), the trihedral provides a large backscattered RCS relative to its physical size (particularly advantageous in environments having strong clutter sources), and has a broad main lobe in its backscatter pattern in both azimuth and elevation, making it relatively easy to orient the trihedral for maximum backscatter return to the radar. 


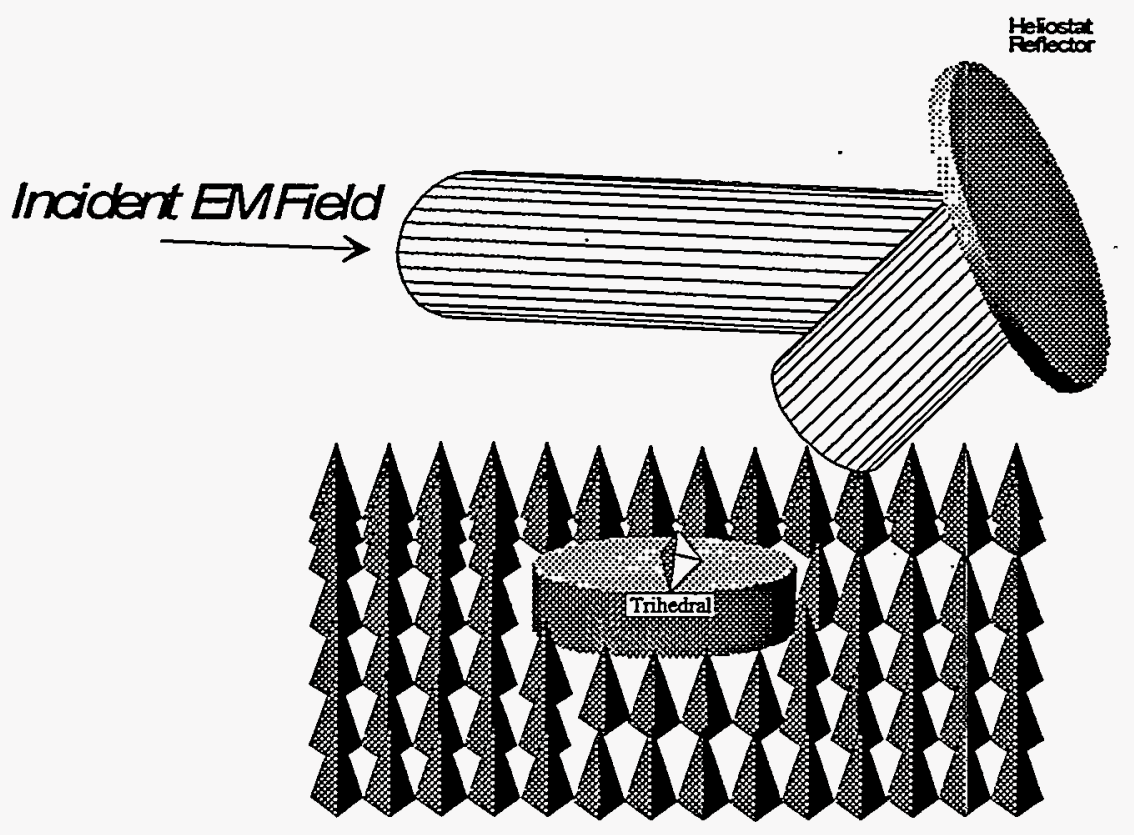

Figure 10.Reference target measurement setup used in calibration of raw data. Calibration standard used was a triangular trihedral corner reflector.

As alluded to previously, prior to measurement of the selected distributed target sample, a few "test" targets were selected and imaged to test the dynamic range and quantitative accuracy of the measurement system. These test targets included an assortment of trihedral corner reflectors and small spheres, as well as somewhat more exotic objects such as a plastic model airplane having a wingspan of approximately 64 inches. The measurements were made by covering the sample holder with sheets of radar absorbing material, and placing the test targets directly onto the absorber. The raw data was calibrated according to the expression given in (4), and was then processed using conventional signal processing to form focused, twodimensional inverse synthetic aperture radar images of the test targets. Two examples are shown in Figures 11 and 12 below.

The theoretical radar cross section of a $3 / 8$ " sphere varies from $-37 \mathrm{dBsm}$ to $-47 \mathrm{dBsm}$ over the frequency range of $8.0-16.0 \mathrm{GHz}$, with the average value being approximately $-40 \mathrm{dBsm}$. The two-dimensional FFT used to create the image shown in Figure 11 essentially averages the amplitude values of the scattered return over the range of measured frequencies. The measured amplitude of the backscattered return from the $3 / 8$ " sphere shown in the plot of Figure 11 (although difficult to distinguish in black and white) is about $-43 \mathrm{dBsm}$, a difference from the average theoretical value that is consistent with the field probe data shown previously. This figure also shows that the return from a 3/16" diameter sphere was able to be detected, and that this sphere had a backscatter level approximately $20 \mathrm{~dB}$ higher than the clutter level, emphasizing the large dynamic range which has been achieved with the system. Figure 12 simply illustrates the ability of an ISAR system to create radar backscattered images which can be similar to the optical image of the test object. 


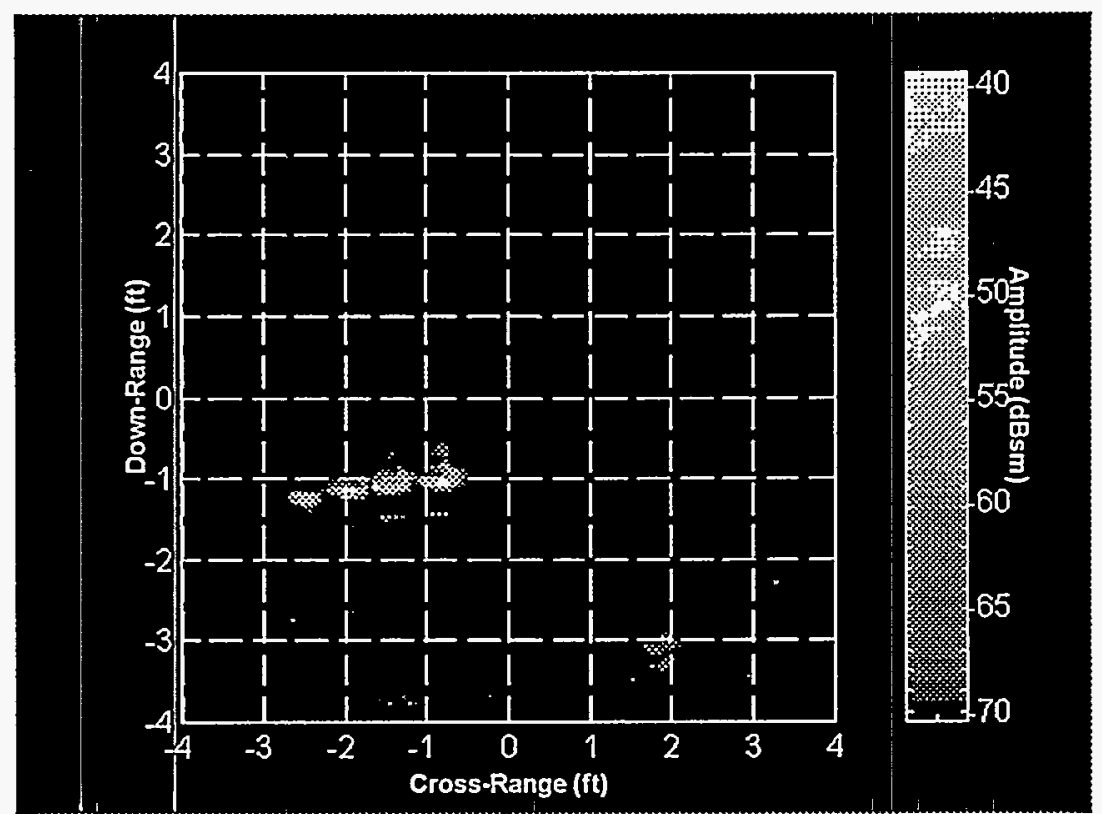

Figure 11. Two-dimensional image of four spheres having diameters of 3/16", 1/4", 5/16", and $3 / 8^{\prime \prime}$, illustrating the resolution capability and dynamic range of the SNL compact range CCD measurement system. The spheres are physically arranged in a line, and are mounted on absorber sheets placed on top of the sample holder. Stepped-frequency bandwidth is $8.0-16.0 \mathrm{GHz}$.

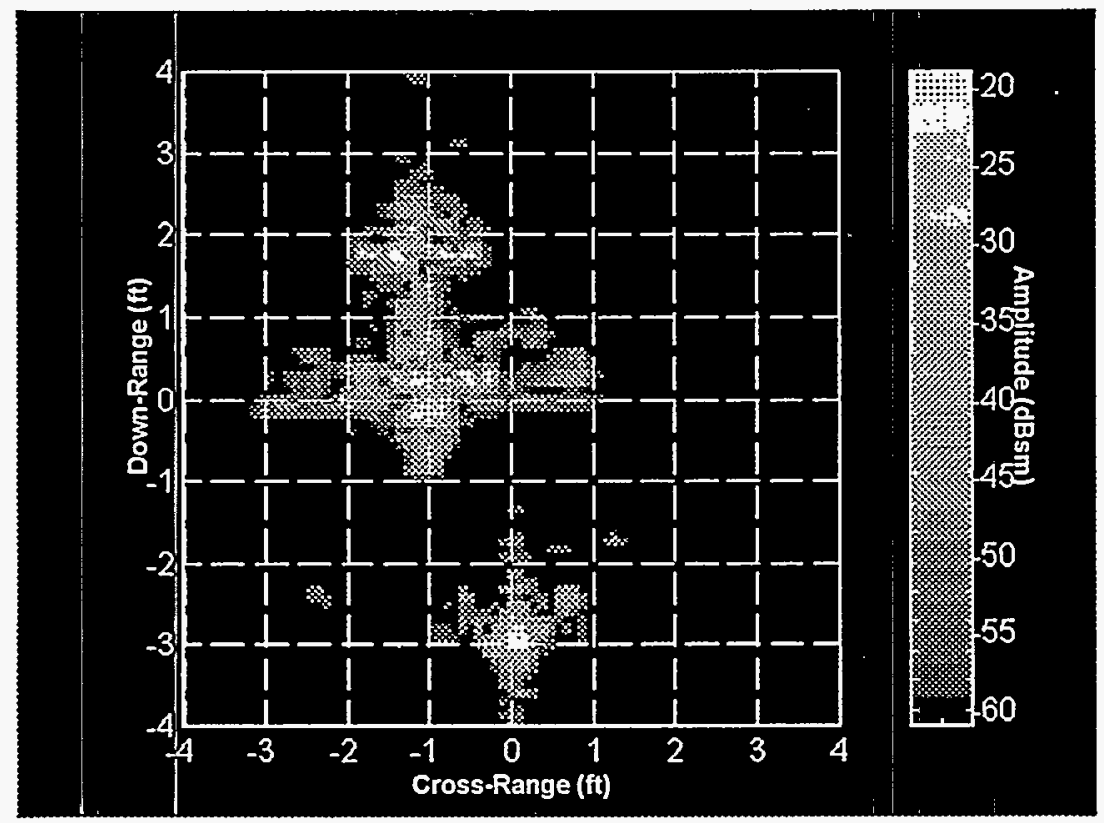

Figure 12. Two-dimensional image of plastic model airplane and corner reflector trihedral mounted on absorber on top of target sample holder. Stepped-frequency bandwidth is $8.0-12.0 \mathrm{GHz}$. 


\subsection{CCD Image Formation}

After calibrating the raw data according to the expression given in (4), conventional signal processing is used, as stated above, to form focused, two-dimensional inverse synthetic aperture radar (ISAR) images of the backscattered return from the distributed target. As previously mentioned, this results in data which is equivalent to that which can be acquired with a circular spotlight SAR. The formation of a coherent change detection (CCD) image requires that a pair of two-dimensional, complex images be generated which may then be "compared" in some fashion. The pair of images result from measuring first an "undisturbed" or pristine distributed target sample, followed by a measurement of the same target sample, but which has been perturbed in some manner. The object of the comparison is thus to determine how the target has been changed, or in other words, how it has "decorrelated."

In standard communication theory, "correlation" is the degree to which the variation of two variables is harmonized [12]. The lack of correlation, or "decorrelation," between a pair of two-dimensional radar cross section images is a result of differences in the amplitude and phase values between the images. 'In the case of a realistic SAR platform collecting CCD image data, decorrelation could occur as a result of several factors, including noise (thermally generated or otherwise), motion of the target, uncompensated motion of the SAR platform, image registration errors, calibration errors, etc. [13]. In the SNL compact range environment, many of these decorrelation sources have been eliminated or minimized. For example, the radar itself is stationary, so that there is no need for motion compensation hardware or software. The motion of the target is controlled by a precision motorized turntable and positioner controller, such that elevation and azimuth aspect angles of the target are controlled (and repeatable) to within $0.05^{\circ}$. Image registration errors (at the wavelength of operation) are therefore negligible. The measured CW noise floor of the RF hardware is approximately $-120 \mathrm{dBm}[14]$; as a result, the noise floor of the compact range system as a whole is limited by the clutter sources that exist in the anechoic chamber. The anechoic chamber incorporates an environmentally-controlled atmosphere and is highly stable, such the clutter sources which do exist remain coherent (and thus correlated) much longer than the time necessary to make the measurements required to generate a pair of 2-d complex images for the purposes of forming a CCD image. By far the most significant source of decorrelation, then, results from the purposeful disturbing of the target sample, and the effect of applying a comparison, or decorrelation detection, algorithm to two complex images reflects this fact.

Decorrelation detection of two complex images could potentially be accomplished in a number of ways. Three methods were investigated as a part of this study, all of which were found to be successful in detecting the decorrelated portion of the distributed target sample: (1) coherent subtraction, (2) coherent ratioing, and (3) use of the maximum likelihood estimate of correlation algorithm [13]. The second method, that of coherent ratioing, consisted of simply performing a complex ratioing of the two images on a pixel-by-pixel basis. Although adequate in detecting the decorrelated portions of the target sample, this method did not appear to be as robust as the other two methods (possibly as a result of the color maps used to represent the data), and so will not be discussed further. 
The second method, coherent subtraction, is analogous to the vector subtraction of clutter data $\left(B K G_{\text {test }}\right)$ from test target data $\left(T G T_{\text {test }}\right)$ as described above. However, as opposed to doing the subtraction on the raw data, the vector subtraction was performed on a pixel-bypixel basis after the two 2-d images were formed. This method is particularly effective, since subtraction of pixels of two images which are correlated (unchanged) gives a result of "zero" (or in other words a value that is roughly equal to the effective noise floor of the measurement system), while subtraction of totally decorrelated pixels (having nearly equal backscattered cross section amplitudes, but opposite phases) will result in a value that is approximately doubled in amplitude (increase of $3 \mathrm{~dB}$ ) relative to the original image pixel values. Partially decorrelated pixels will have values ranging somewhere between these two extremes. The effect in the final image is thus to cause the decorrelated pixels to stand out, while the correlated pixels become embedded in the background noise.

The third method investigated, maximum likelihood estimate of correlation, is given by [13]

$$
\gamma=\frac{\left|\sum_{i} \sum_{j} g_{i j}^{*} h_{i j}\right|}{\sqrt{\left.\left|\sum_{i} \sum_{j}\right| g_{i j}\right|^{2} \cdot \sum_{i} \sum_{j}\left|h_{i j}\right|^{2} \mid}}
$$

where

$$
\begin{aligned}
g_{i j}= & \text { the first registered complex image for the } \mathrm{i}^{\text {th }} \text { range index and the } \mathrm{j}^{\text {th }} \text { azimuth } \\
& \text { index; } \\
h_{i j}= & \text { the second registered complex image for the } \mathrm{i}^{\text {th }} \text { range index and the } \mathrm{j}^{\text {th }} \text { azimuth } \\
& \text { index. }
\end{aligned}
$$

The summations are over the number of "looks" or pixel samples used in range and azimuth for the estimation. A more detailed explanation of this algorithm is given in [15] and [16], but essentially it is designed to perform an optimal estimation of the magnitude of the normalized correlation of two images, and is very similar to the coefficient of correlation as given in standard texts on communication theory [12]. This algorithm is the same one that has been applied to image data gathered with a variety of operational SAR platforms, and has been found to be equally (if not more) effective when applied to data measured on the SNL compact range.

Examples of images produced using the coherent subtraction and maximum likelihood estimate of correlation methods are given in the following section. 


\section{CCD DATA COLLECTION}

The final validation of the compact range $\mathrm{CCD}$ data collection configuration was to attempt to create CCD images of a distributed target sample with which successful CCD images had previously been generated from an operational SAR platform. Based on this premise, as well as ease of handling, availability, and economic considerations, the best alternative seemed to be to use gravel for the target sample. "Pea-sized" gravel (stones having diameters of roughly $1 / 4-3 / 8$ inches) was procured and loaded into the sample holder, such that the top surface of the gravel was nominally level with the top edge of the sample holder. The resultant gravel depth was approximately six inches (the remainder of the sample holder being filled with polystyrene and radar absorbing material, as discussed earlier).

\subsection{CCD Image Resolution}

The images created for each measurement set are noted in the test plan below, and are displayed in the appendices. Appendix A consists of the set of images that were processed using coherent subtraction; Appendix B consists of images processed using the maximum likelihood estimate of correlation (5). It will be noticed in the images that the pixel size is not equal to the image resolution. For example, the images resulting from measurements made using the radar parameters specified in Table 1 have resolutions of approximately one inch, while the pixel size is 0.64 inches. This can be explained as follows.

As shown by Mensa [1], the maximum achievable down-range and cross-range resolutions for unwindowed data are given respectively by

and

$$
\Delta R=\frac{c}{2 B}
$$

$$
\Delta x=\frac{c}{2 f \Theta},
$$

where $B$ and $\Theta$ are the effective bandwidth (in $\mathrm{Hz}$ ) and total angular aperture (in radians), and $c$ is the velocity of propagation of the EM field, which in this case is equal to the speed of light. When preparing to perform a gated-CW radar-cross-section measurement on the compact range, the first parameter to be considered, then, is the desired resolution of the final image, since this value will determine the necessary bandwidth and angular extent over which the measurement must be taken. $\Delta R$ and $\Delta x$ are typically chosen to be equal so that the pixel dimensions in the final image will be square. Swept frequency measurements on the SNL compact range are made using a stepped-frequency waveform. The proper frequency and angular step sizes must therefore be carefully chosen in order to insure that the quiet zone, or target space, remains alias free. That is, by choosing the appropriate frequency and angular step increments, undesired noise and multi-path responses outside of the target space can be prevented from folding back on top of the target in the final image. This is also shown in [1], where the unambiguous down-range and cross-range image extents are given by 


$$
R_{E}=\frac{c}{2 \Delta f}
$$

and

$$
x_{E}=\frac{c}{2 f \sin (\Delta \theta)}
$$

where $\Delta f$ and $\Delta \theta$ represent the frequency and angular step size, respectively.

The Knowbell ${ }^{\mathrm{TM}}$ software which is used to process the measured data into two-dimensional, focused images assumes that the total image size is equivalent to $R_{E}$ and $x_{E}$ (these values are assumed to be equal, because of the presumption that the final image utilizes square pixels) [17]. The number of pixels in the image is chosen by using the next highest power of two above the number of test frequencies utilized in the measurement, and is calculated according to the relationship [17]

$$
N_{\text {pixel }}=2^{I N T\left\{1+\left[\frac{\log _{10}\left(N_{\text {freq }}-1\right)}{\log _{10}(2)}\right]\right\}},
$$

where $N_{\text {freq }}$ is the number of measurement frequencies and is given by $\frac{B}{\Delta f}$. The pixel size is simply the image size divided by the number of pixels. Using (8), it is simple to show that the pixel size is given by

$$
\text { Pixel Size }=\frac{c}{2 B} \times \frac{N_{\text {freq }}}{N_{\text {pixel }}}=\Delta R \frac{N_{\text {freq }}}{N_{\text {pixel }}}
$$

Hence, the pixel size will equal the image resolution only when the number of steppedfrequency measurements equals the number of pixels used to form the final image.

Unfortunately, the parameters chosen for this initial set of measurements did not satisfy that condition, such that the pixel size is not equal to the resolution of the image. These two values are therefore listed separately on each figure.

As a final note, it should also be mentioned that, while the unwindowed resolution of each image is equal to $\Delta R$, the image resolution indicated on each figure is actually equal to $1.47(\Delta R)$. This is because Hamming weighting was applied to the frequency domain data during image processing in order to reduce the range sidelobes in the final image. This windowing function results in resolution broadening of $47 \%$ [18], such that the actual resolution of the final image is somewhat degraded from its optimum value.

\subsection{Test Plan for CCD Measurements}

A test plan was created which, it was felt, would allow several aspects of CCD measurement and image formation to be investigated. The test plan used is given below. It should be noted 
that except for the "image resolution" tests, all experiments were performed using the radar parameters specified in Table 1. Also, photographs were taken of the gravel samples both prior to and after measurement of each sample configuration. Generally speaking (excepting gross changes in the gravel surface, such as heavy raking or the application of water to the gravel), the changes made to the gravel could not be detected visually.

\section{CCD IMAGE MEASUREMENT TEST PLAN}

\section{Null Set Experiment}

Intent is to determine level of nominal backscattering from the gravel surface.

(1) Measure the gravel in the sample holder and create a two-dimensional image of the gravel without performing any decorrelation detection processing. (Figure A-2)

\section{Decorrelation Detectability Experiments}

Intent is to determine levels of discernible disturbance of the gravel surface.

(2) Walk across gravel

a) Walk across sample holder in distinct pattern (serpentine path, right angle path, etc.), and retrace steps one time (that is, walk over same path two times), then measure. (Figures A-3, B-1)

b) Walk across sample holder in distinct pattern, but do not retrace steps. Space steps far enough apart that they can be individually resolved in the image, and measure. (Horizontally-polarized images are in Figures A-4 and B-2. Verticallypolarized images are in Figures A-5 and B-3.)

(3) Rake across gravel (single pass of rake across diameter of sample holder).

a) Light raking--barely disturb surface (not visibly apparent), and measure. (Figures A-6, B-4)

b) Medium raking--overturn majority of rocks in raking path, but leave no rake tine marks (not visibly apparent), and measure. (Figures A-7, B-5)

c) Heavy raking--Fully embed rake.tines in rocks; leave visible indentations in gravel, and measure. (Figures A-8, B-6)

(4) Discrete rock displacement

a) Within approximately 8 inch diameter circles in the sample holder, individually pick up the following number of rocks, replace them in the same (as nearly as possible) position, and measure: (i) 10 rocks, (ii) 20 rocks, and (iii) 40 rocks. (Figures A-9, B-7).

b) Within approximately 8 inch diameter circles in the sample holder, individually pick up the following number of rocks without replacing them, and measure: (i) 5 rocks, (ii) 10 rocks, (iii) 20 rocks, and (iv) 40 rocks. (Figures A-10, B-8) 
Image Resolution Experiments

Intent is to examine the effect of varying the image resolution (pixel size) in CCD images.

(5) Walk across sample holder in distinct pattern, but do not retrace steps. Space steps far enough apart that they can be individually resolved in the image. Measure this configuration four different times, using (equivalent) down-range and cross-range resolutions of:
a) 8.7 inches (pixel size $=3.8$ ") (Figures A-11, B-9);
b) 4.3 inches (pixel size $=2.3$ ") (Figures A-12, B-10);
c) 2.2 inches (pixel size $=1.2$ ") (Figures A-13, B-11);
d) 1.1 inches (pixel size $=0.64$ ") (Figures A-4, B-2).

\section{Moisture Effects Experiment}

Intent is to examine the effect of moisture in decorrelation of $\mathrm{CCD}$ images.

(6) Moisture test

a) Make a set of tire tracks in the gravel (approximately 24 inches apart and $~ 3$ inches deep) and measure. (Figures A-15, B-12)

b) After performing the measurement described in (a), evenly sprinkle 2 gallons of water across the entire sample holder, and immediately re-measure (DO NOT remeasure test background). (Figures A-17, B-13)

c) While allowing the water to "completely" evaporate (until the gravel becomes visually dry), re-measure the gravel at periodic intervals (e.g., "half-dry", "mostlydry", "completely-dry", etc.). (Figures A-18,19,20, B-14,15,16)

\section{Radar Stability Experiment}

Intent is to provide an indication of the level of coherency maintained by the compact range $\mathrm{CCD}$ measurement system as a function of time.

(7) Time interval test

a) Make a set of tire tracks in the gravel (approximately 24 inches apart and $\sim 3$ inches deep) and measure; (Figures A-21, B-17)

b) Leaving all hardware continuously powered-up, re-measure the tire tracks each day for a period of 4 days, using the original test background measurement, calibration file, etc. (Figures A-22,23,24,25, B-18,19,20,21)

\subsection{Discussion of Results}

This purpose of this section is to briefly discuss the outcome of each set of experiments, as well as to highlight some of the more interesting results and findings.

\section{Null Set Experiment}

The intent of this experiment was to determine the level of nominal backscattering from the gravel surface. The maximum level of backscatter from the gravel was measured to be -26.3 $\mathrm{dBsm}$; however, the vast majority of discretized scatterers had backscatter values ranging 
from $-50 \mathrm{dBsm}$ to $-30 \mathrm{dBsm}$. Also of interest was the fact that the background clutter level outside of the sample holder area was in general less than $-70 \mathrm{dBsm}$.

\section{Decorrelation Detectability Experiments}

The intent of this set of experiments was to determine the level of disturbances of the gravel surface that could be detected by the compact range CCD measurement system. Both walking and raking across the surface of the gravel were easily detectable: It was of particular interest that the average levels of backscattered return displayed on the raked-gravel CCD images were basically the same, regardless of whether the surface was "lightly raked" or "heavily raked." The main discernible difference was the width of the disturbance displayed on the image (presumably due to a larger number of pebbles being disturbed with the heavier raking).

Also of note was the outline of the boot print that was resolved in Figure B-2 (processed using the maximum likelihood estimate of correlation algorithm), an amazing indication of the power of performing coherent measurements.

The first discrete rock displacement images (Figures A-9, B-7) show the 10-rock displacement sample as providing a return that is "brighter" than the 20-rock sample, and nearly as "bright" as the 40-rock sample. The reason for this is that the person performing the test dropped a pebble or two from the "10 rock sample" when trying to pick up the pebbles individually with his fingers (which then of course displaced a few other pebbles when they hit), before he realized that tweezers would need to be used for the procedure. The results of the "discrete rock displacement" test were therefore somewhat invalidated, but were indeed effective in indicating the level of sensitivity of the system. The set of discrete rock removal images (Figures A-10, B-8) also highlighted the ability of the CCD images to detect very minor disturbances of the gravel surface, as demonstrated by the fact that the mere removal of five pebbles resulted in a detectable change.

\section{Image Resolution Experiments}

The intent of these experiments was to examine the effects of varying the image resolution (pixel size) in the CCD images. As expected, as the resolution was decreased, the image definition correspondingly decreased, such that the footprints became more difficult to distinguish. Coherent subtraction seemed to result in a better image at the lowest resolution tested (8.7") than could be obtained with the maximum likelihood estimate of correlation, although at the higher resolutions the results were comparable.

\section{Moisture Effects Experiment}

The intent of this set of experiments was to examine the effect of moisture in the decorrelation of CCD images. The device used to create the tire tracks in the gravel is shown in Figure A14. The application of moisture to the surface of the gravel resulted in a near total decorrelation of the CCD image, as expected. The volume of water applied to the gravel was minimal-just enough to simply wet (as opposed to "soaking") the entire surface. As the moisture evaporated, the original disturbance (tire tracks) of the surface again became apparent in the processed images. The gravel was only allowed to dry for a period of one day 
( $\sim 24$ hours), before the final "dry" configuration was measured. At this point in time, the top surface of the gravel was visually dry. (A set of photographs showing the amount of moisture that was visible on the gravel throughout the period of time during which the measurements were made is given in Figure A-16.) However, it is felt that underlying moisture which was not visible contributed to a lack of correlation in the final image, resulting in tire tracks that were less distinct than those seen in the original image of the tire tracks (made before applying moisture to the surface of the gravel). It is further felt that, were the gravel allowed to dry for an extended period of time, the tire tracks would become nearly as distinctly defined as in the original image. This is perhaps an item for further study.

\section{Radar Stability Experiment}

The intent of these experiments was simply to understand the level of coherency that can be expected to be maintained by the compact range $\mathrm{CCD}$ measurement system as a function of time. The images indicate that the system remained very stable over the five-day period of the experiment, with no substantive changes appearing in the CCD images until the final day (Friday). This information was also applicable to the "moisture effects" experiment, in that it indicates that essentially none of the decorrelation that appeared in the CCD images of the wet gravel during the one-day period over which the moisture experiment was conducted was due to instability in the measurement system.

\section{PROPOSAL FOR FOLLOW-ON CCD MEASUREMENTS}

Due to the level of success which has been achieved with the initial set of CCD measurements at the SNL compact range measurement facility, it is felt that follow-on measurements have the potential of contributing significantly to the body of knowledge available to the scientific community involved in CCD image generation and processing. The following three areas in particular are felt to warrant further investigation:

(1) Measurement of a number of other "sample" types, which could include

- soil,

- sand,

- grass (sod),

- asphalt;

(2) Environmental conditions affecting CCD image quality, such as

- rain/moisture,

- wind,

- temperature (diurnal heating, etc.);

(3) Mechanics of CCD image formation, including

- sensitivity to azimuth/elevation angular positioning errors (i.e., how does image quality degrade as a result of navigation errors; does this sensitivity vary with a change in image resolution?),

- effect of non-square pixels on image quality, 
- polarization effects (are there any advantages to using cross-polarized backscattered returns as opposed to concentrating solely on copolarized returns?),

- image quality as a function of signal-to-noise and signal-to-clutter ratios.

Additionally, there are a few "human factors" issues with regard to the manner in which the measured data is presented which should also be resolved. This would include the selection of a "standardized" color map for data presentation (number and intensity of colors used to plot images), as well as a "standardized" display format (information included on an image, etc.).

\section{CONCLUSION}

This report has described the folded compact range configuration that has been developed at the SNL antenna and radar cross section measurement facility as a means of performing indoor, environmentally-controlled, far-field simulations of synthetic aperture radar coherent change detection measurements. This compact range configuration provides the capability to perform wideband, coherent measurements of distributed target samples, producing twodimensional images having resolutions varying from less than 1.0 inch up to approximately one foot.

The report has also described the initial set of coherent change detection measurements made with the system. These measurements of gravel samples have been highly successful, and have demonstrated the viability of the folded compact range concept in simulating SAR CCD measurements.

It is felt that follow-on measurements have the potential of contributing significantly to the body of knowledge available to the scientific community involved in CCD image generation and processing, and that this tool will be a significant aid in the research and development of change detection methodologies. 


\section{REFERENCES}

[1] D. L. Mensa, High Resolution Radar Cross-Section Imaging, Artech House, Norwood, MA, 1991.

[2] N. C. Currie, ed., Radar Reflectivity Measurement: Techniques \& Applications, Artech House, Norwood, MA, 1989.

[3] "Model 5752 Test Data," Scientific Atlanta, Atlanta, GA, March 1988.

[4] E. F. Knott, et. al., Radar Cross Section, Artech House, Norwood, MA, 1985.

[5] M. I. Skolnik, Introduction to Radar Systems, 2nd Edition, McGraw-Hill Book Company, New York, 1980.

[6] E. B. Joy, et. al., "Monostatic Near-Field Radar Cross-Section Measurement," Proceedings of the Antenna Measurement Techniques Association, Oct. 29-31, 1985, Melbourne, FL, pp. 24-1 - 24-11.

[7] S. Brumley, "Indoor RCS Measurement Ranges," lecture notes, Denmar, Inc., 1989.

[8] Science Applications International Corporation, Facet Development for a Faceted Stretched-Membrane Dish by SAIC, SAND91-7008, October 1991.

[9] C. A. Balanis, Advanced Engineering Electromagnetics, John Wiley \& Sons, Inc., New York, 1989.

[10] G. T. Ruck, ed., Radar Cross Section Handbook, vol. 2, Plenum Press, New York, 1970.

[11] "RCS FR959 \& DataPro Technical Reference Manual," Ver. 1.3, Flam \& Russell, Inc., Horsham, PA, June 1993.

[12] B. P. Lathi, Modern Digital and Analog Communication Systems, CBS College Publishing, New York, 1983.

[13] D. L. Bickel, W. H. Hensley, Design, Theory, and Applications of Interferometric SAR for Topographic Mapping: Spaceborne SAR LDRD Final Report, SAND report, to be published.

[14] "HP8530S Measurement System Technical Description," Flam \& Russell, Inc., Horsham, PA, June 1992.

[15] J. P. Burg, et. al., "Estimation of Structured Covariance Matrices," Proceedings of the $I E E E$, vol. 70, no. 9, September 1982, pp. 963-974. 
[16] P. H. Eichel, et. al., "Interferometric Processing of Coherent Spotlight Mode SAR Images," Proceedings of the Interferometric SAR Technology and Applications Symposium, April 13-14, 1993, Ft. Belvoir, VA; pp. 253-274.

[17] "FR Imaging Calculator, Version 2.0, Installation Instructions, Operating Information, \& Technical Notes," Flam \& Russell, Inc., Horsham, PA, October 1993.

[18] J. L. Eaves and E. K. Reedy, eds., Principles of Modern Radar, Van Nostrand Reinhold, New York, 1987. 
Intentionally Left Blank 


\section{APPENDIX A}

CCD Images Processed Using Coherent Subtraction 
Intentionally Left Blank

A-2 


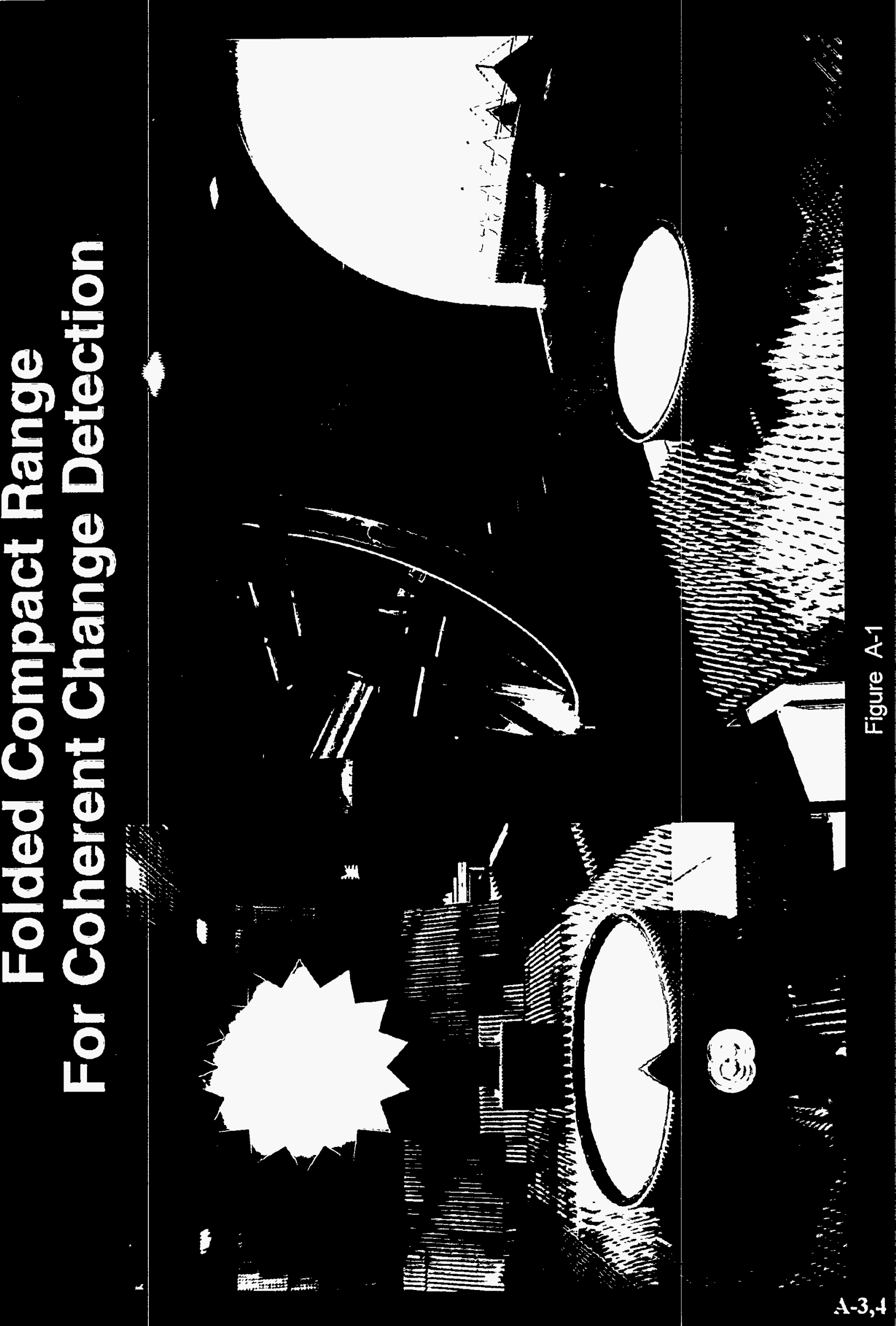




\section{Retraced \\ Footsteps}

(No decorrelation detection)

Azimuth Rotation

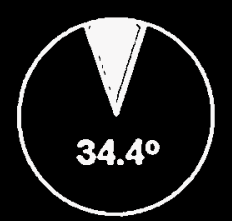

I File: CON5NBK

1 Chirp BW: $8 \mathrm{GHz}$

Center Freq.: $12 \mathrm{GHz}$

Pol.:HH

$X$ Weighting: Hamming

aY Weighting: Hamming

almage Resolution: 1.1 in.

aXV Pixel Size: 0.64 in.

口Processing Method: None

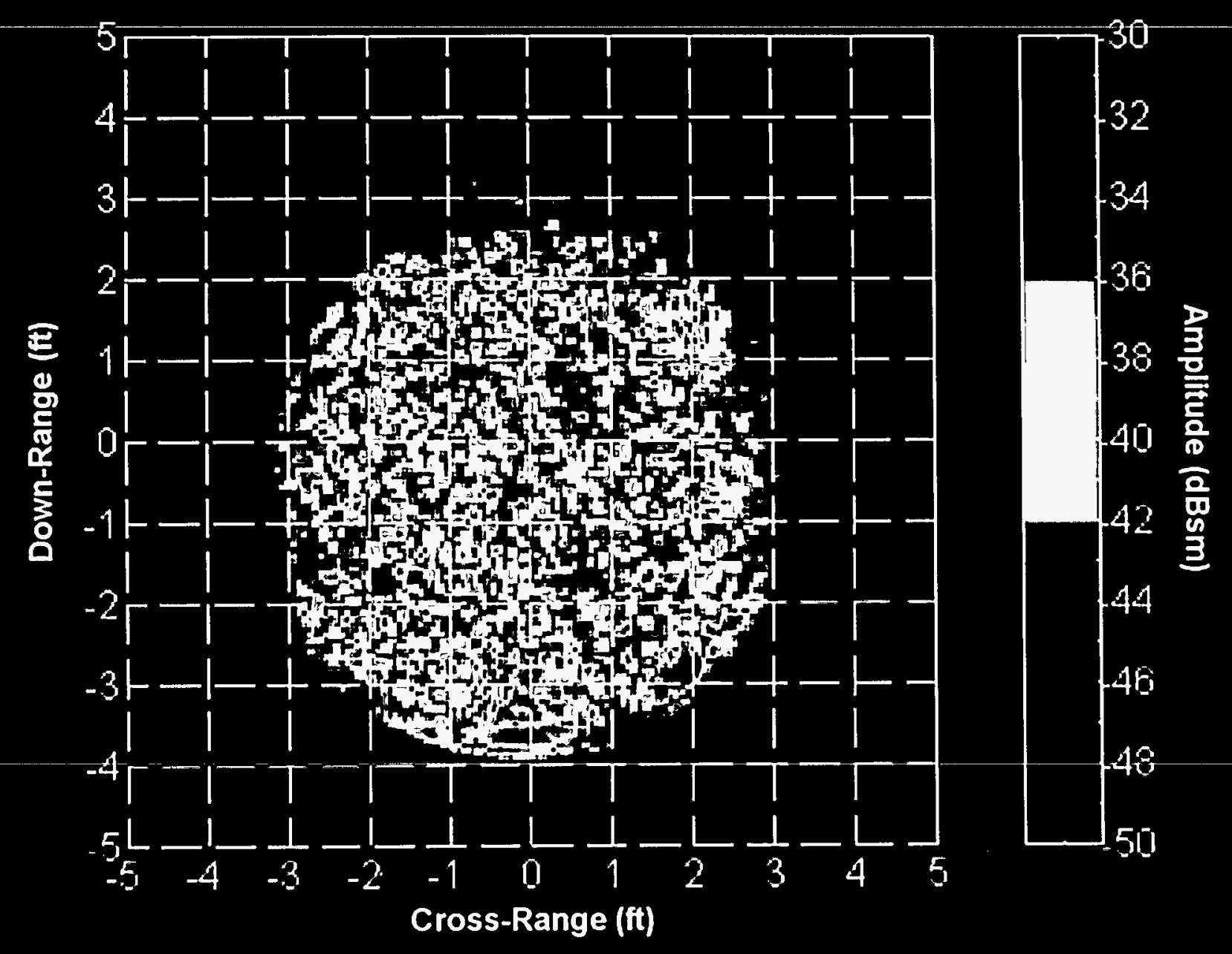

ba

Figure A-2 


\section{Retraced Footsteps}

\section{Azimuth Rotation}

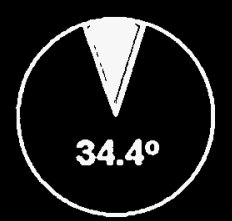

-File: CON5

Chirp BW: $8 \mathrm{GHz}$

center Freq.: $12 \mathrm{GHz}$

Pol.:HH

¿X Weighting: Hamming

$\llcorner Y$ Weighting: Hamming

almage Resolution: 1.1 in.

XY Pixel Size: 0.64 in.

aProcessing Method:

Subtraction

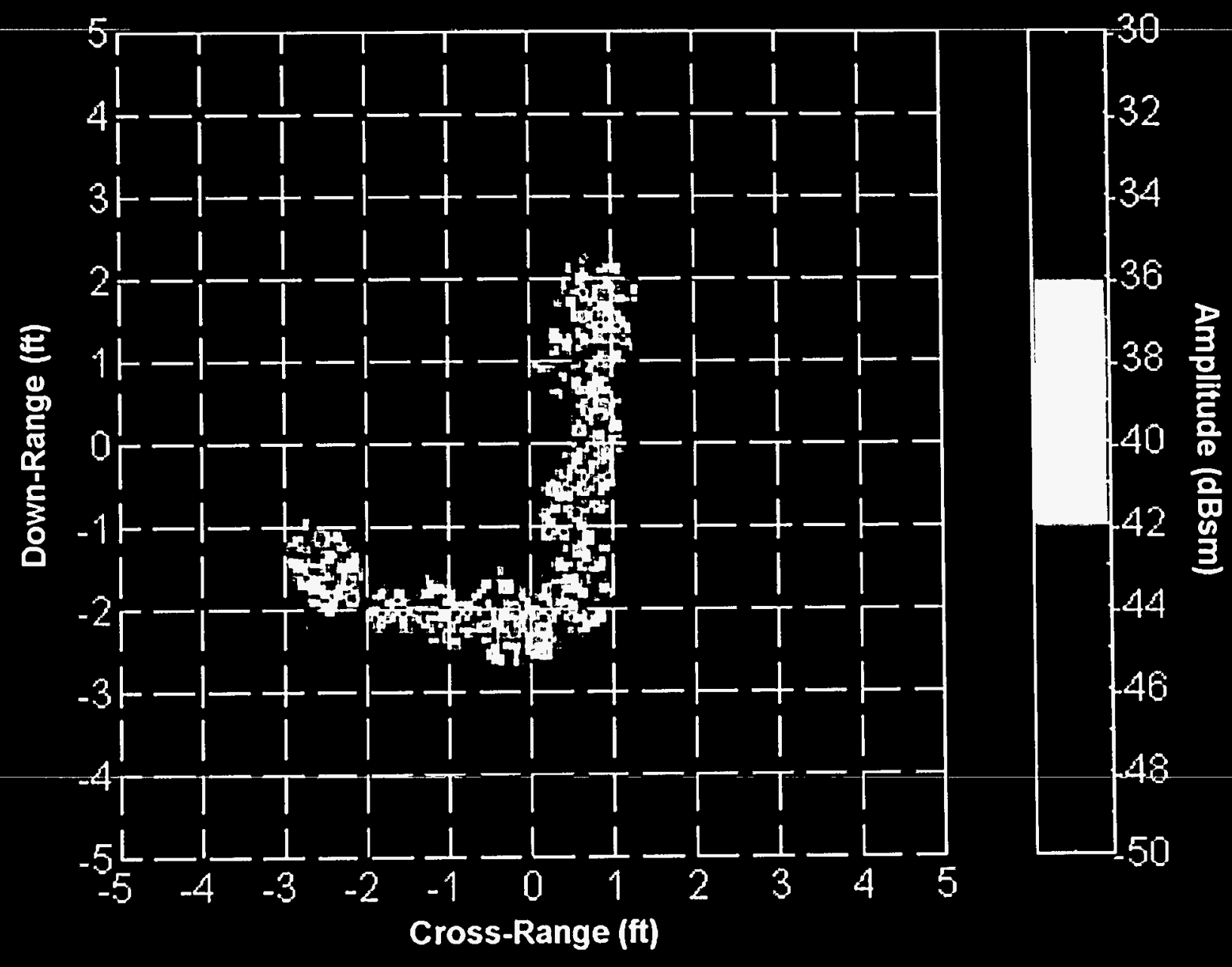

$\underbrace{2}_{-\infty}$

Figure A-3 


\section{Amplitude (dBsm)}

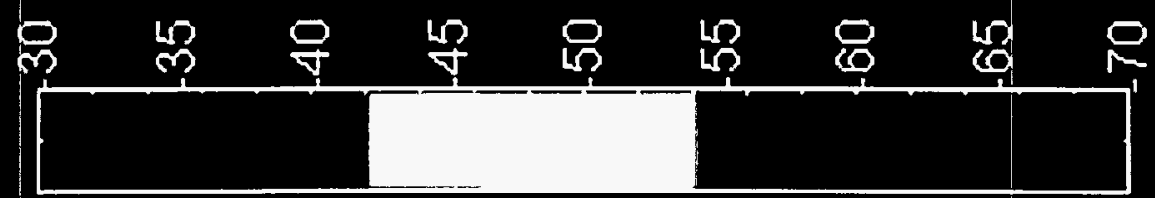

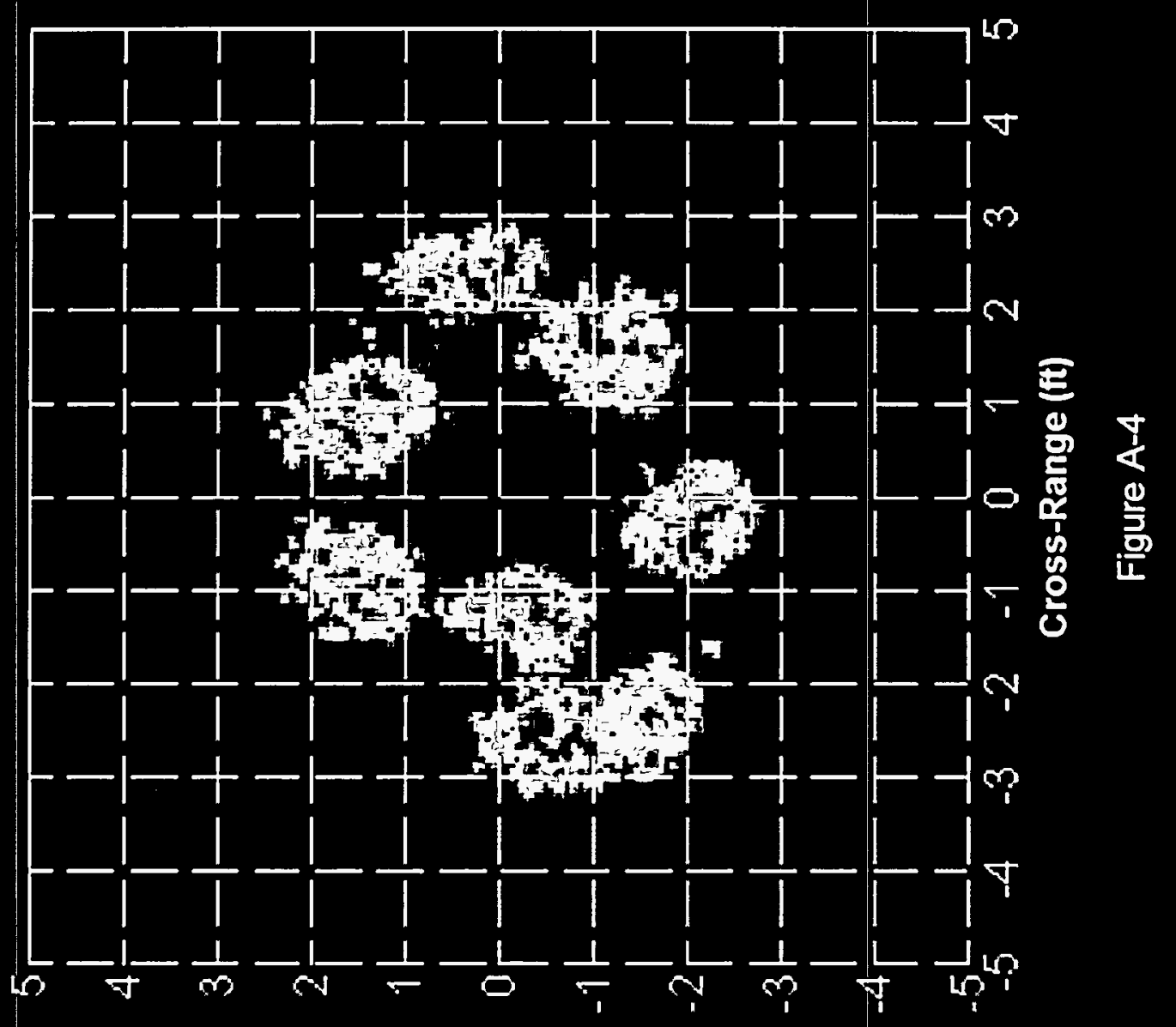

(y) әбиеу-имо

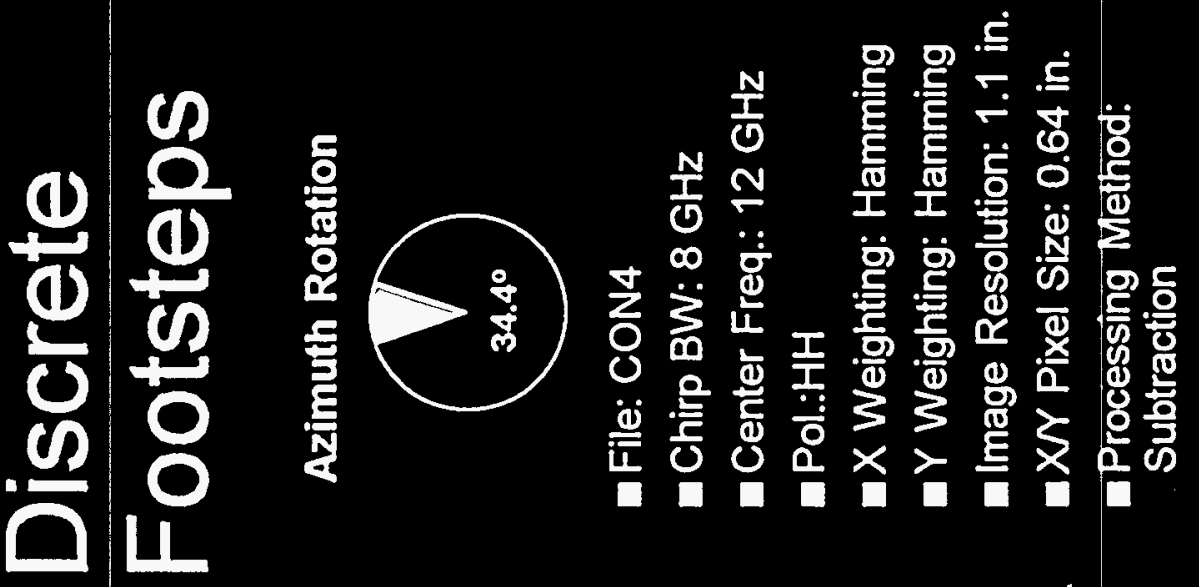




\section{Discrete Footsteps}

Azimuth Rotation

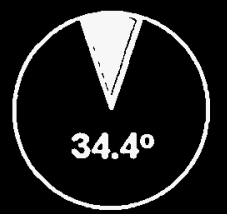

aFile: CON4

Chirp BW: $8 \mathrm{GHz}$

Center Freq.: $12 \mathrm{GHz}$

Pol.:W

$\square X$ Weighting: Hamming

aY Weighting: Hamming

- Image Resolution: 1.1 in.

aXY Pixel Size: 0.64 in.

-Processing Method:

Subtraction

总
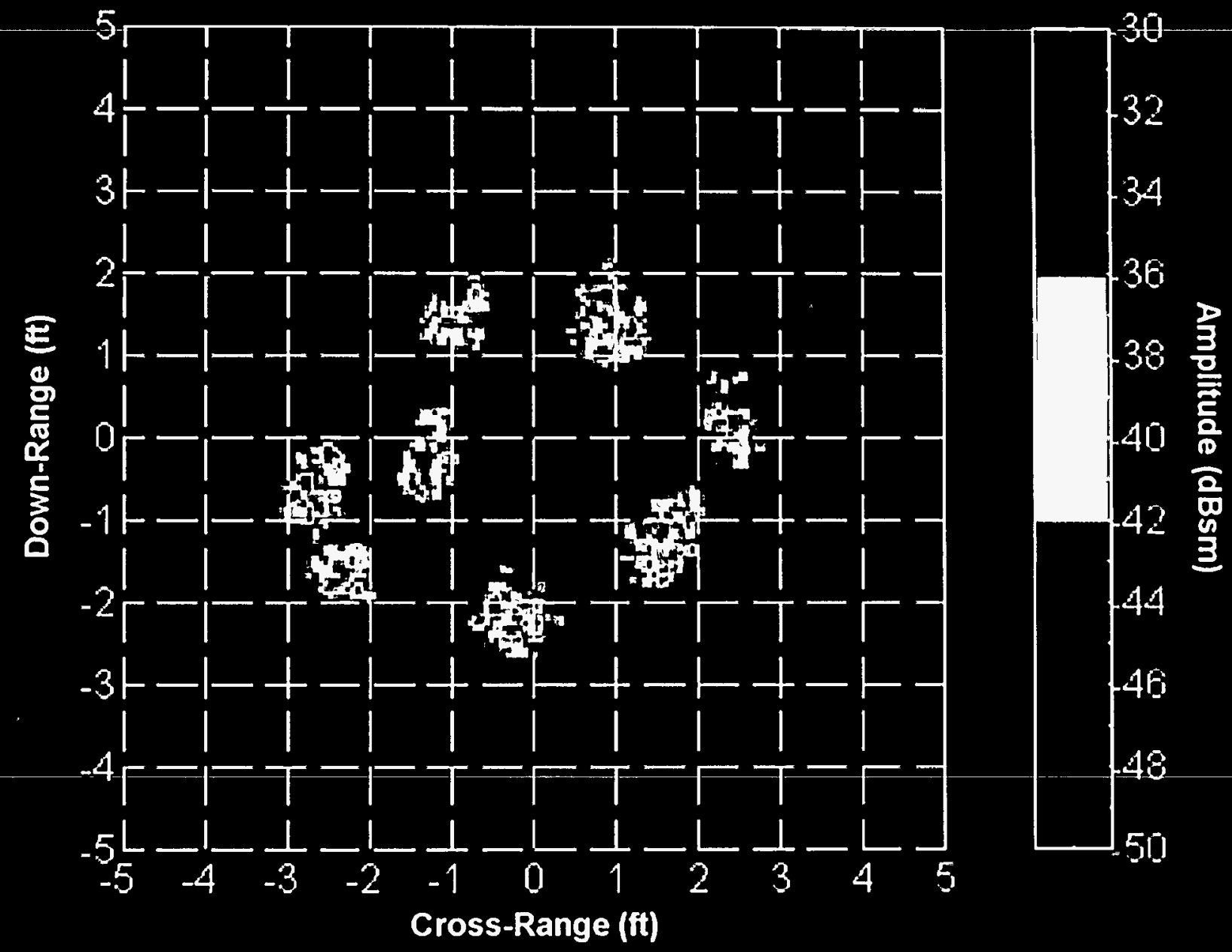

Figure A-5 
Amplitude (dBsm)

m

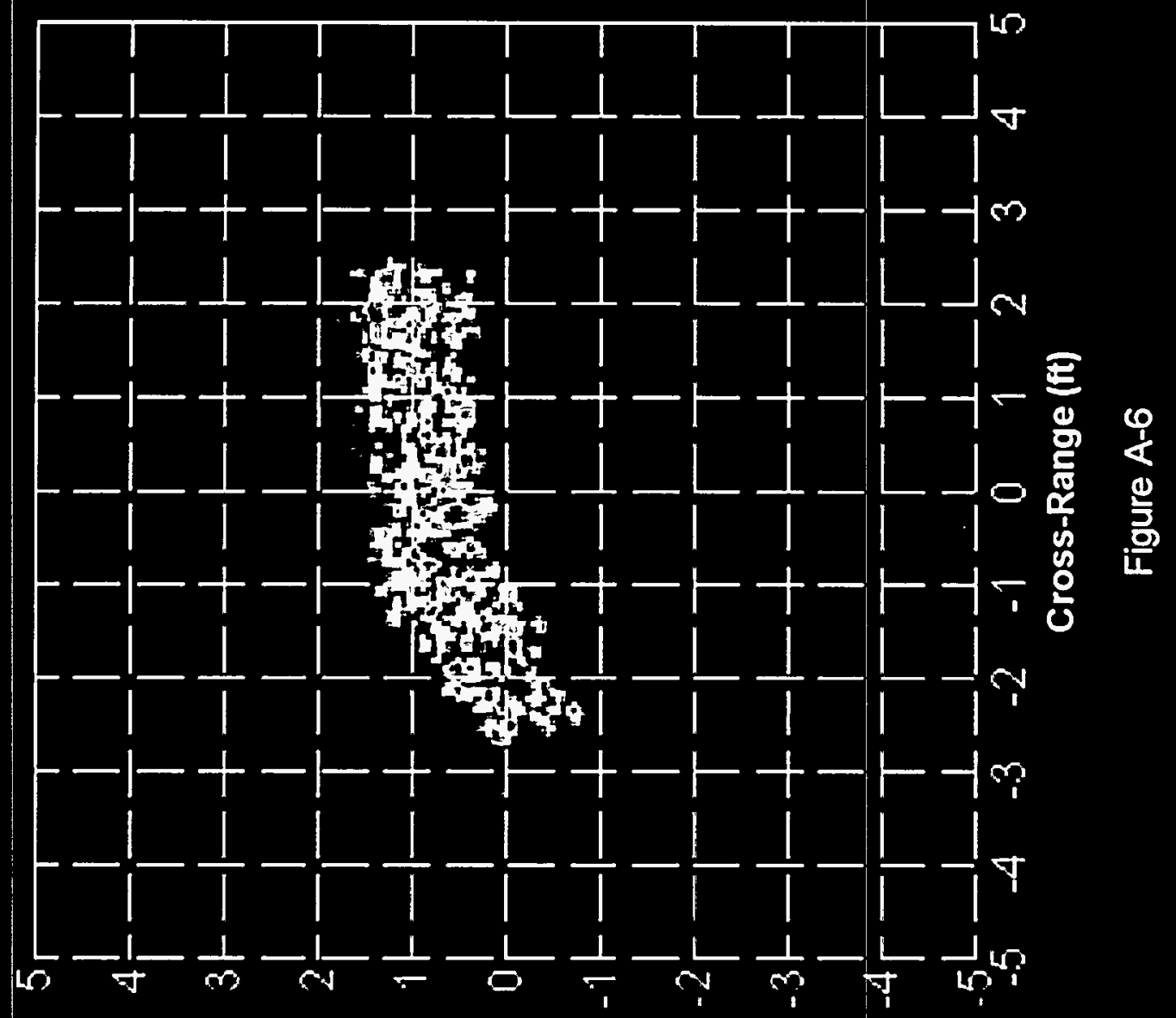

(y) әбиеу-имо

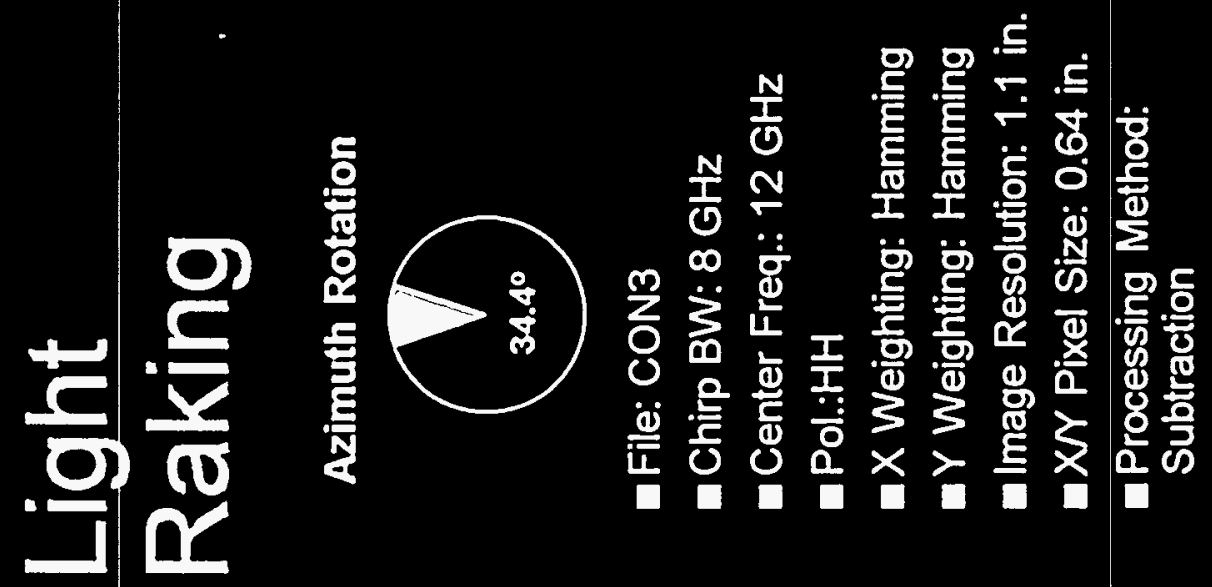




\section{Amplitude (dBsm)}

m ले ले m

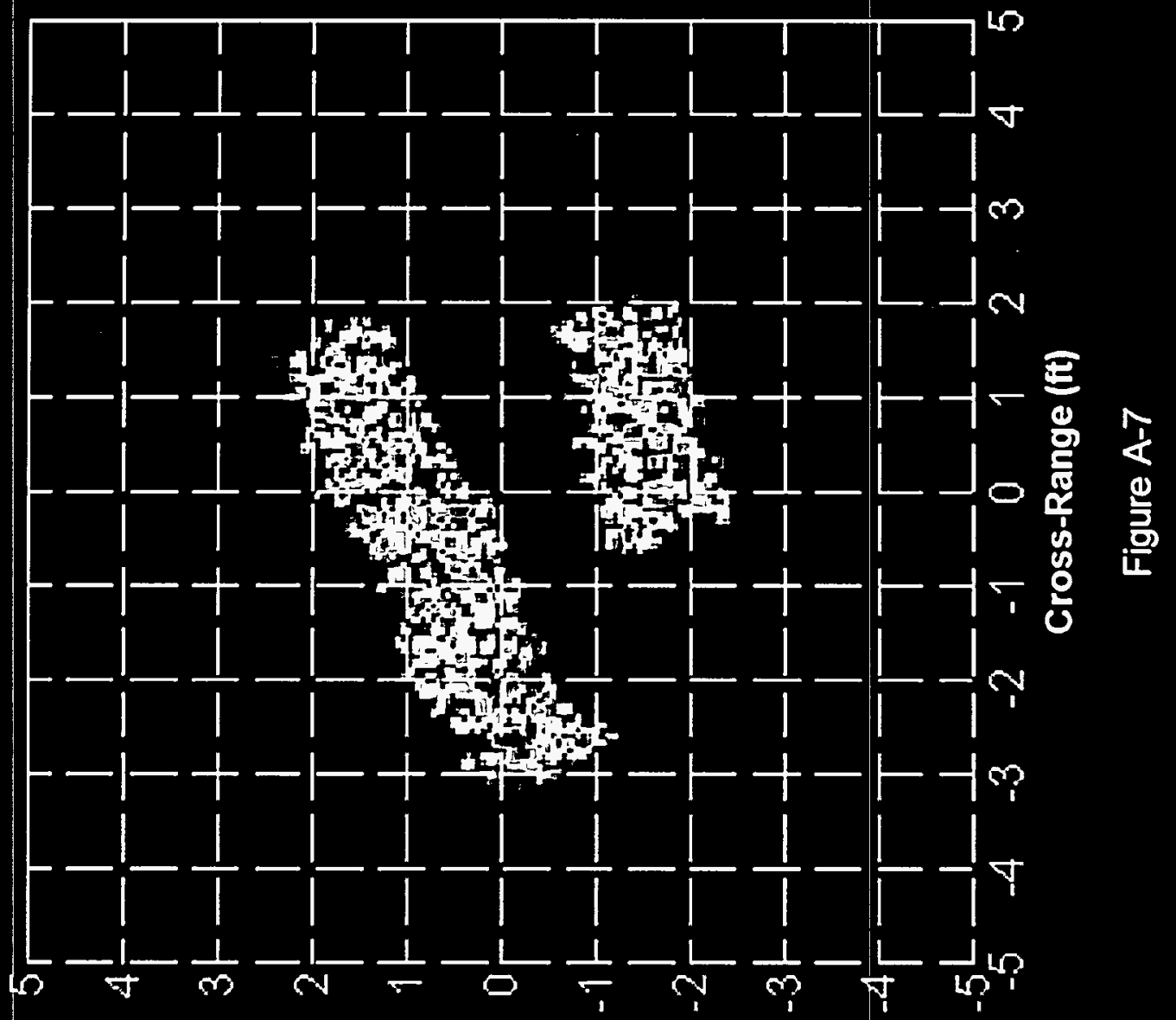

(y) әбuey-umog 


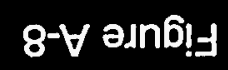

(y) afuey-ssod?

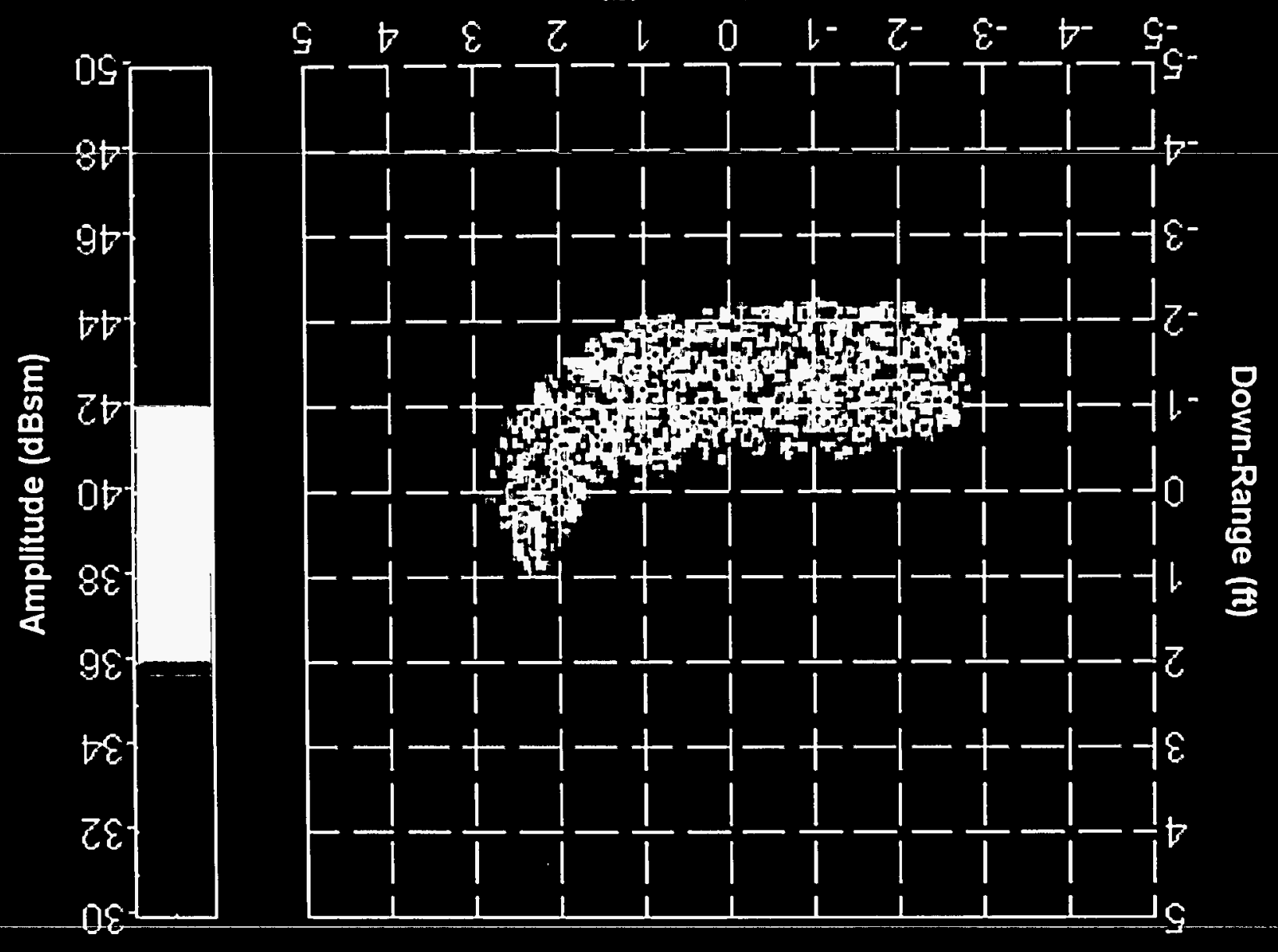

uopjengns :poulew bulssasold 'u! t90 :ez!s lex!d NX u! LL :uognnosey abeup

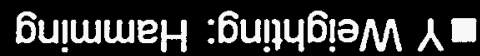

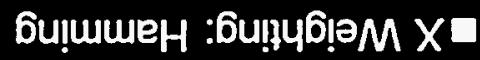
$\mathrm{HH}:$ :jod

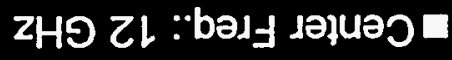
zHอ 8 :Mg dıy० LNOO :레드

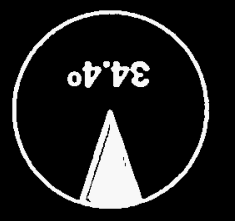

uognejoy ynnulzy

bulyey миеәH 
Amplitude (dBsm)

ले ले ल

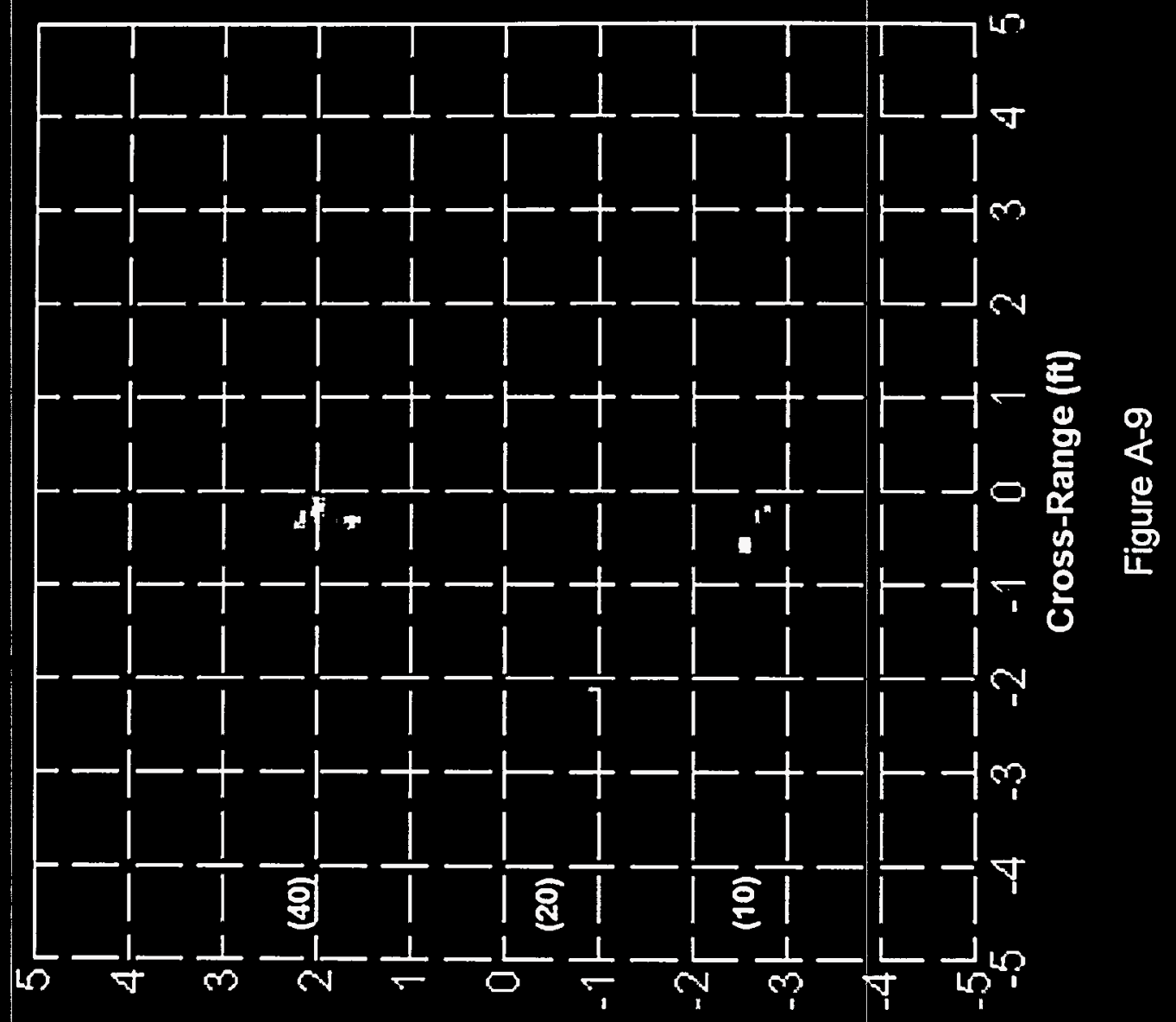

(y) sकिuey-umog
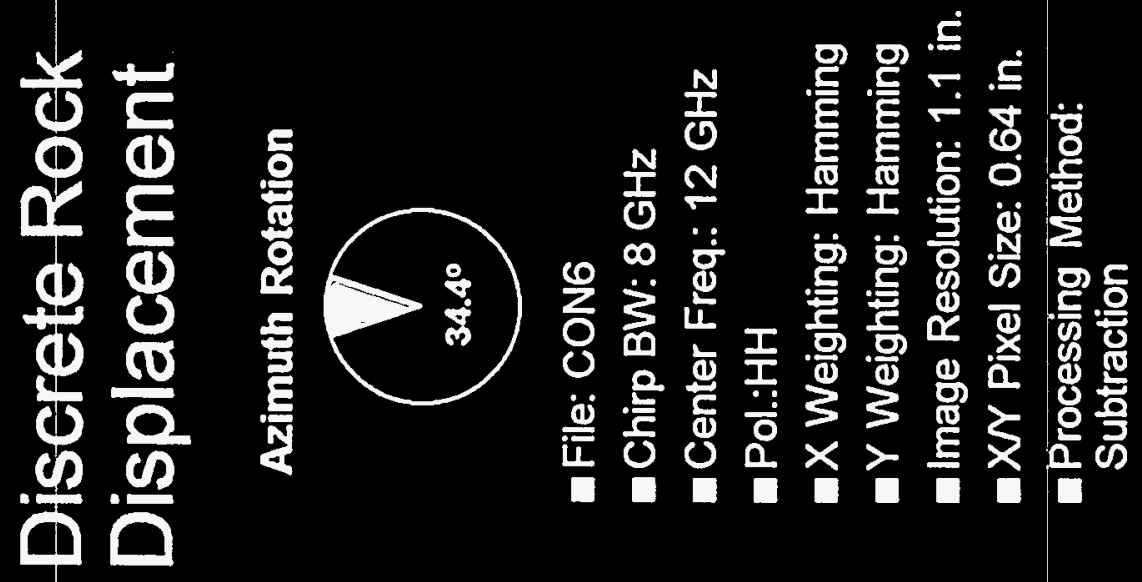


\section{Discrete Rock Removal}

Azimuth Rotation

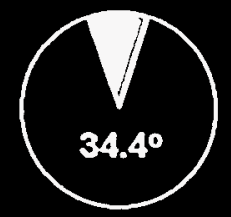

口File: MV2

Chirp BW: $8 \mathrm{GHz}$

-Center Freq:: $12 \mathrm{GHz}$

Pol.HH

$X$ Weighting: Hamming

$Y$ Weighting: Hamming Image Resolution: 1.1 in.

aXY Pixel Size: 0.58 in.

aProcessing Method:

Subtraction

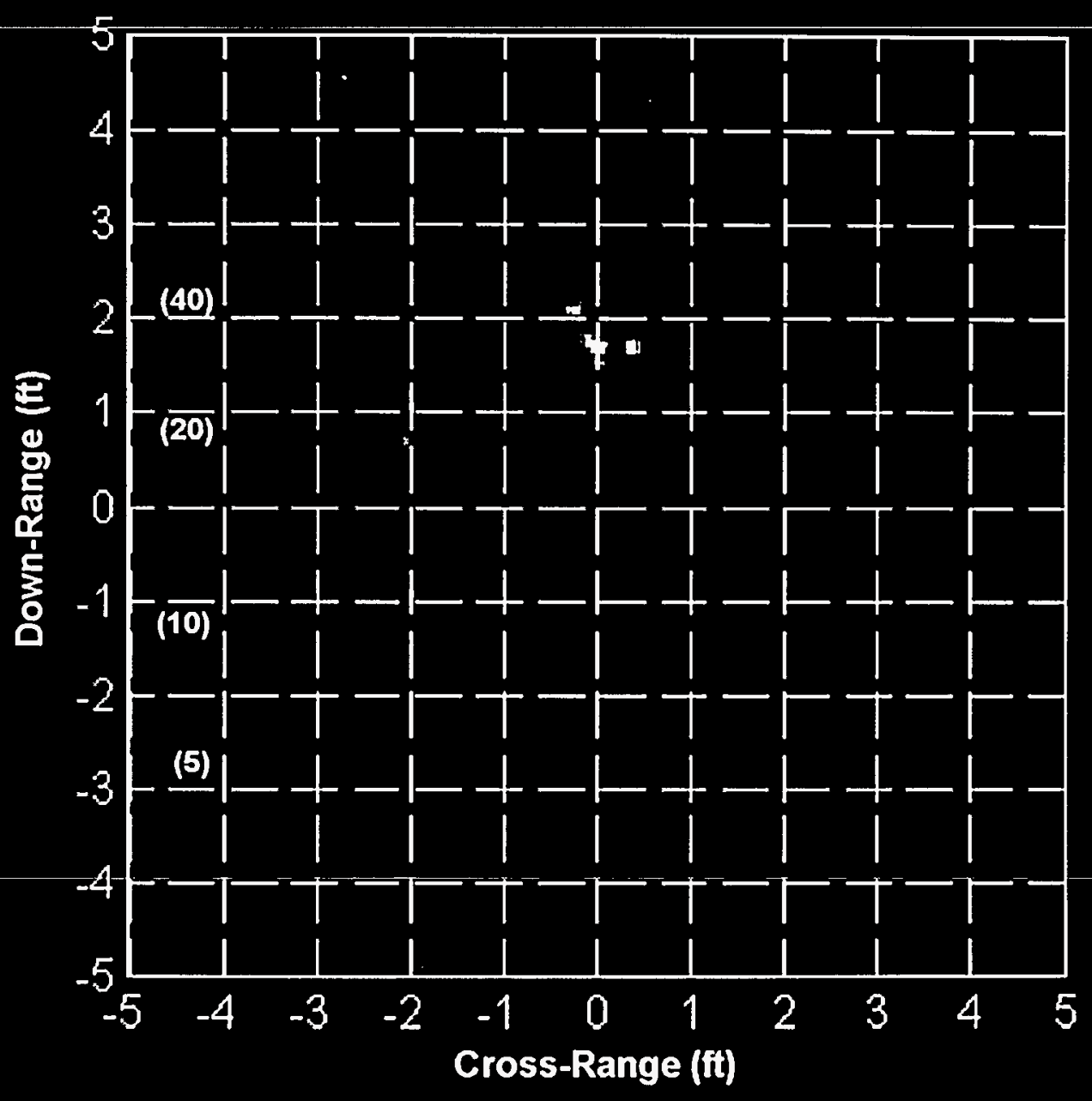




\section{Amplitude (dBsm)}
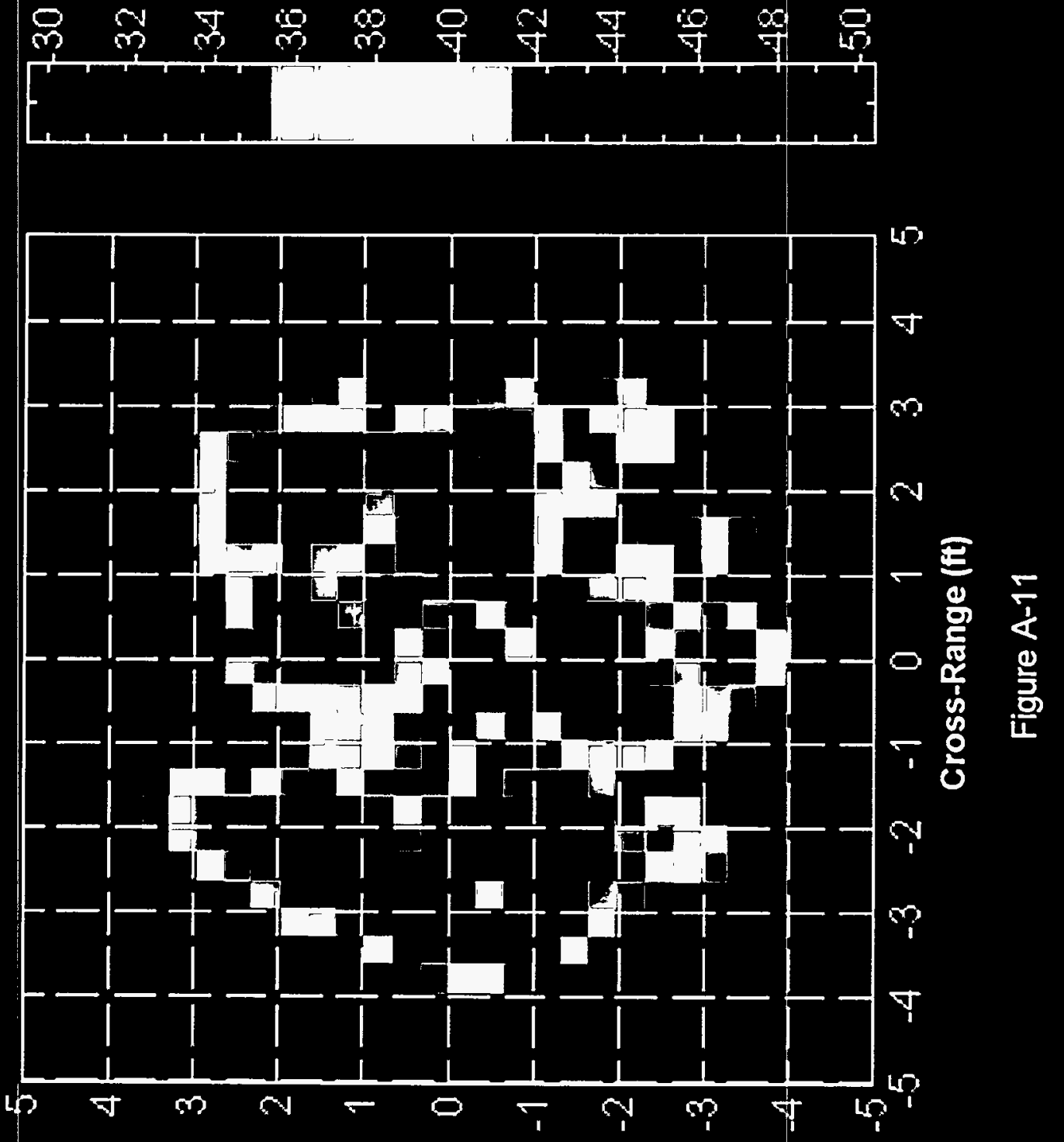

(y) әбиey-uмо

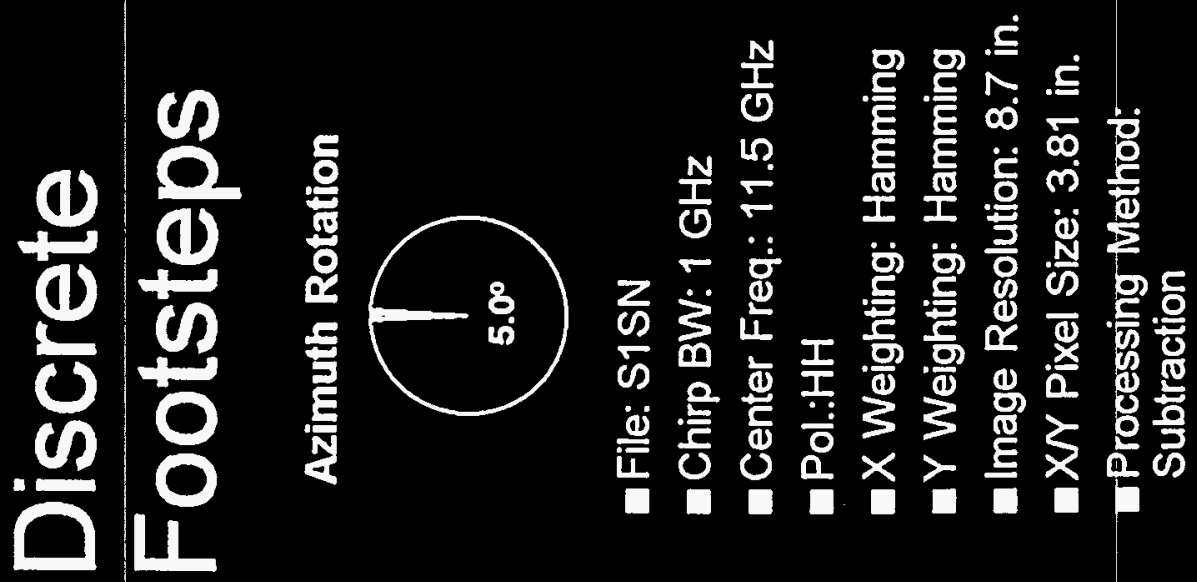




\section{Discrete \\ Footsteps}

Azimuth Rotation

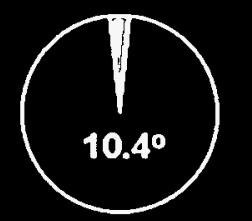

$\square$ File: S2SN

- Chirp BW: 2 GHz

a Center Freq.: $11.0 \mathrm{GHz}$

aPol.:HH

口X Weighting: Hamming

-Y Weighting: Hamming

Image Resolution: 4.3 in.

aXY Pixel Size: 2.34 in.

aProcessing Method:

Subtraction
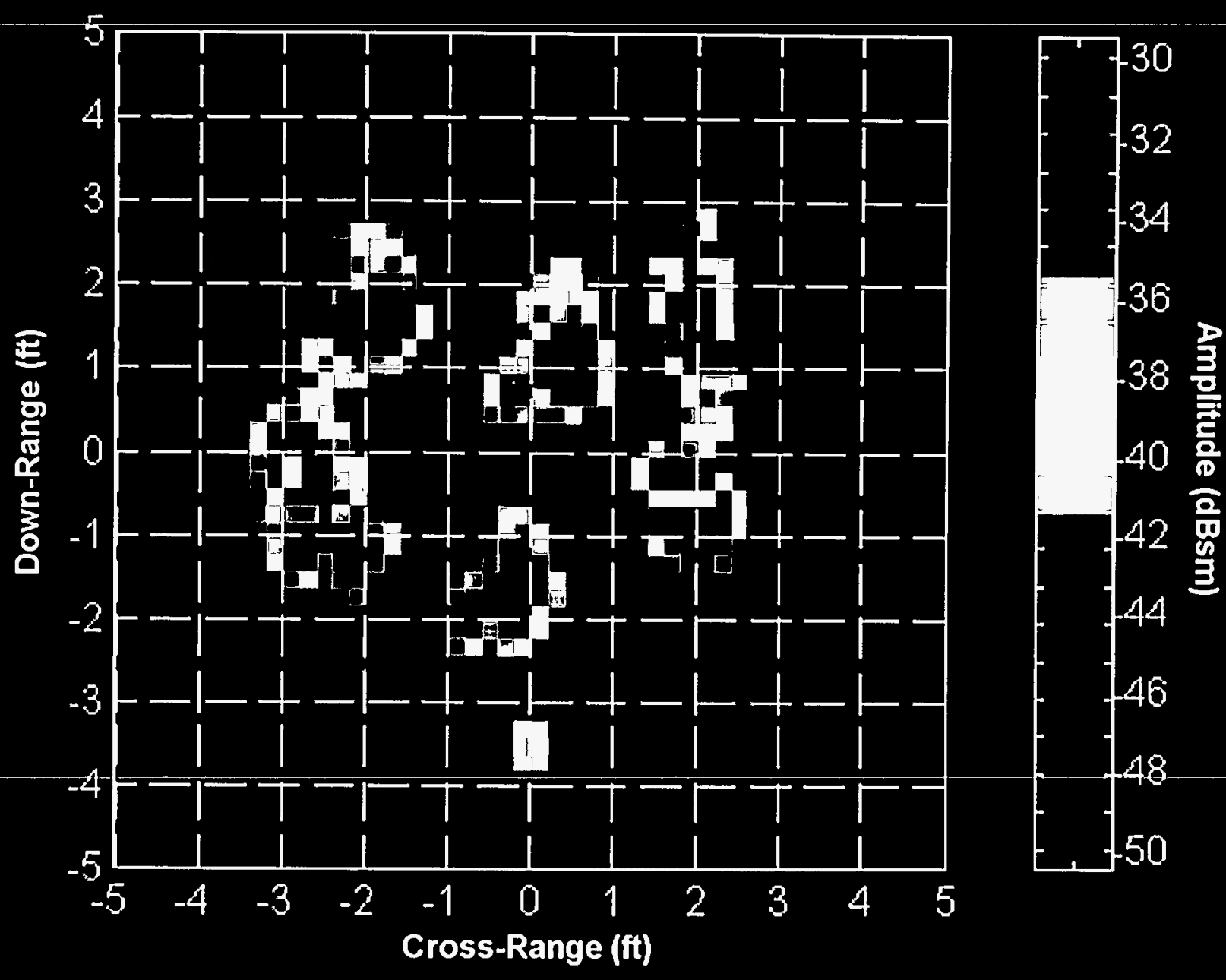

党

Figure A-12 


\section{Discrete \\ Footsteps}

\section{Azimuth Rotation}

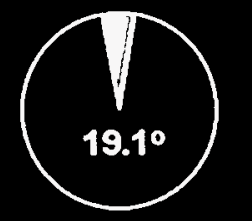

aFile: S4SN

-Chirp BW: $4 \mathrm{GHz}$

| Center Freq.: $12.0 \mathrm{GHz}$

口Pol.:HH

$\ulcorner\mathrm{X}$ Weighting: Hamming

$\ulcorner Y$ Weighting: Hamming Image Resolution: 2.2 in.

aXY Pixel Size: 1.16 in.

aProcessing Method:

Subtraction

$\frac{1}{N}$
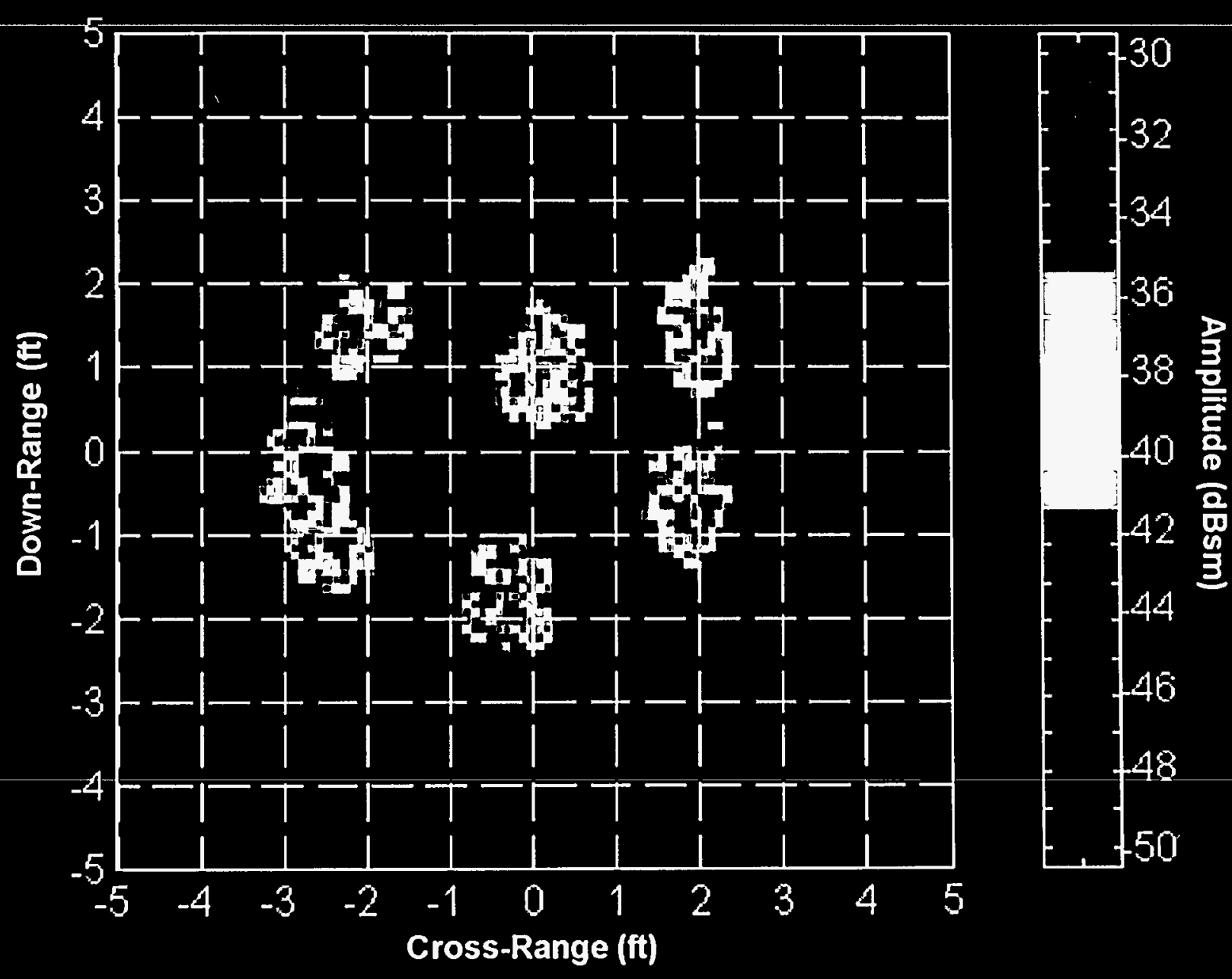

Figure A-13 


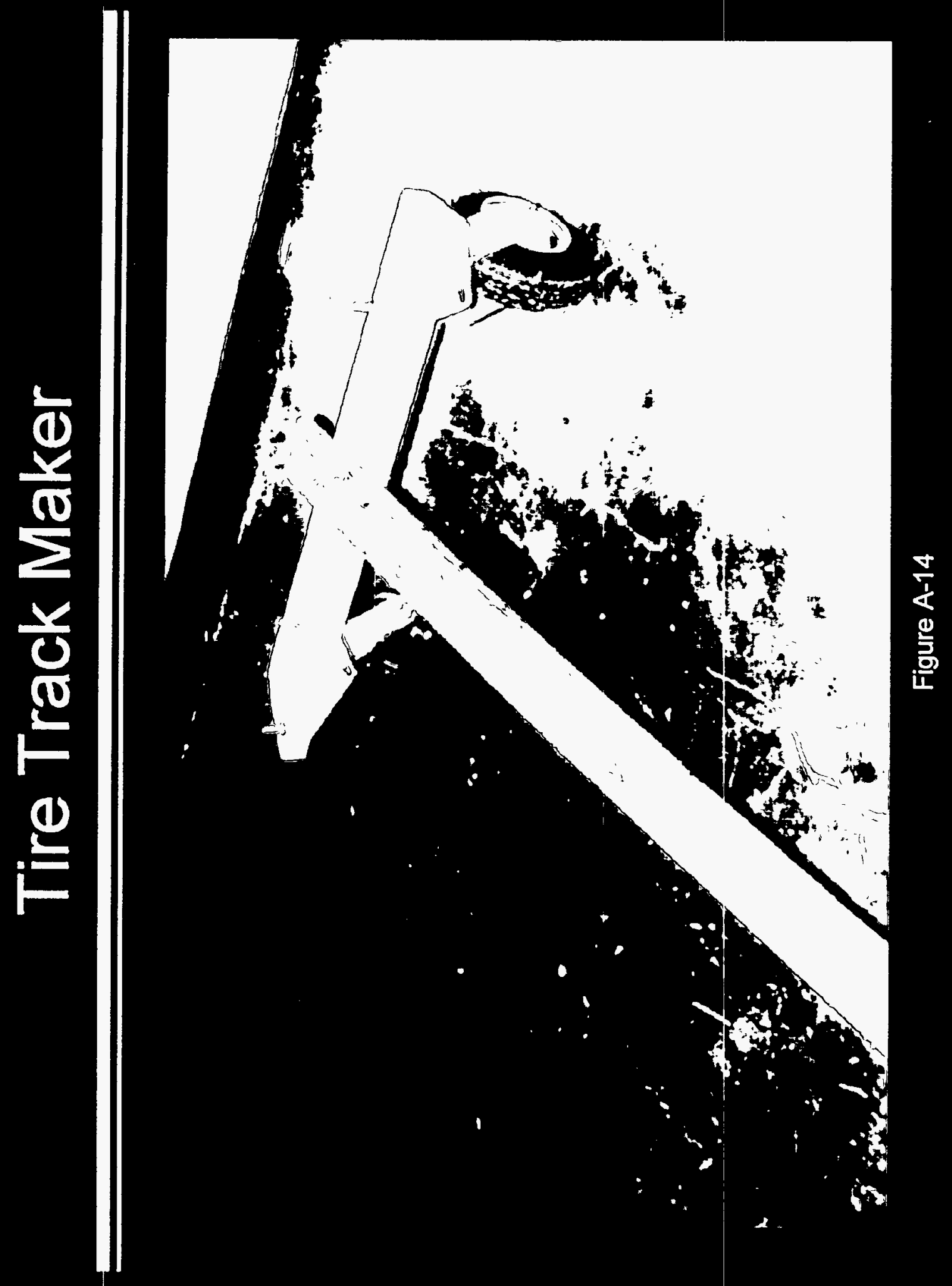


Tire Tracks

Azimuth Rotation

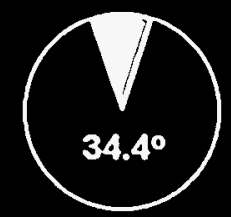

-File: CON8

$\ulcorner$ Chirp BW: $8 \mathrm{GHz}$

I Center Freq.: $12 \mathrm{GHz}$

aPol.:HH

I $X$ Weighting: Hamming

aY Weighting: Hamming

Image Resolution: 1.1 in.

$\square X Y$ Pixel Size: 0.64 in.

Processing Method:

Subtraction

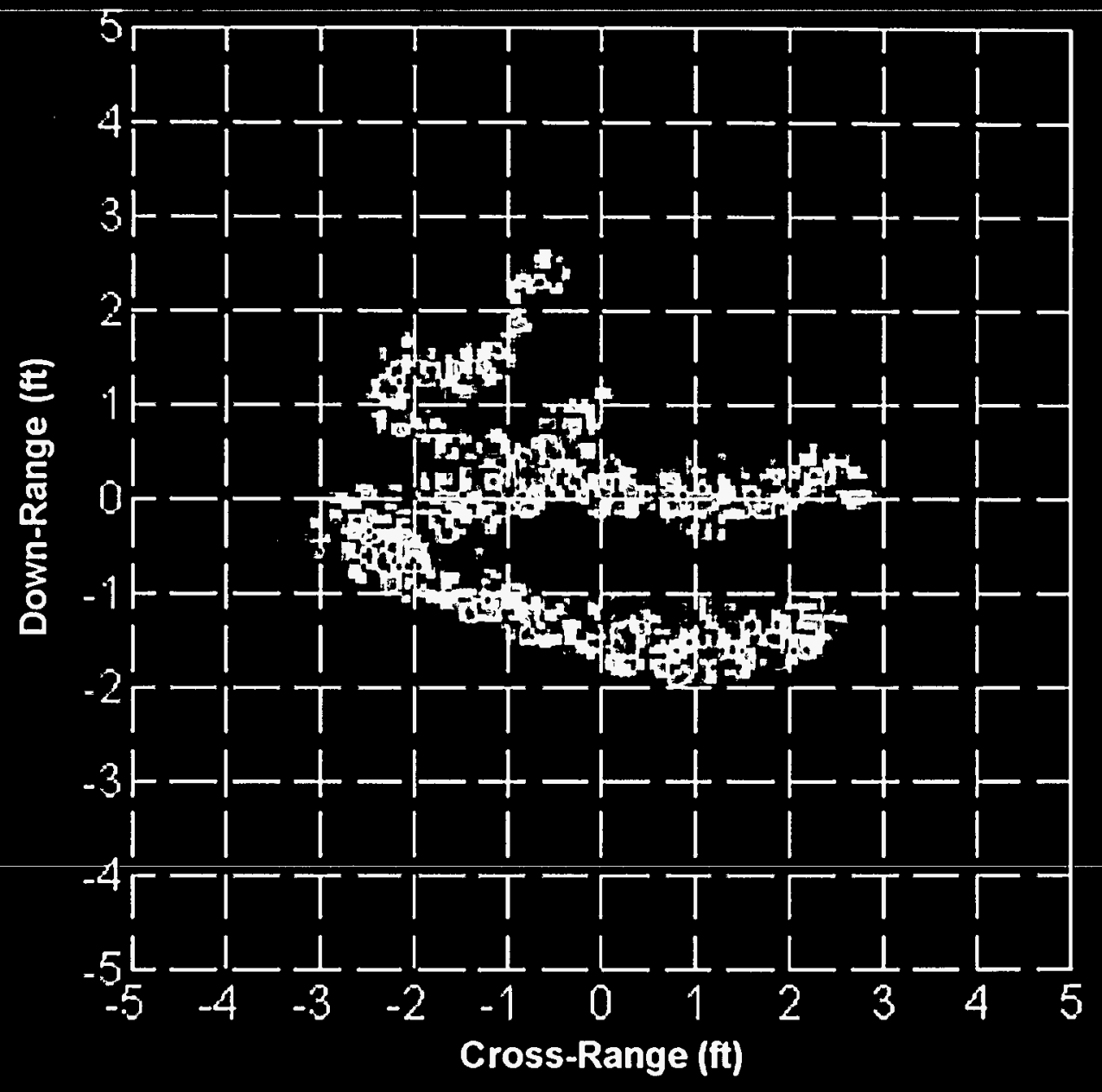

总

Figure A-15 

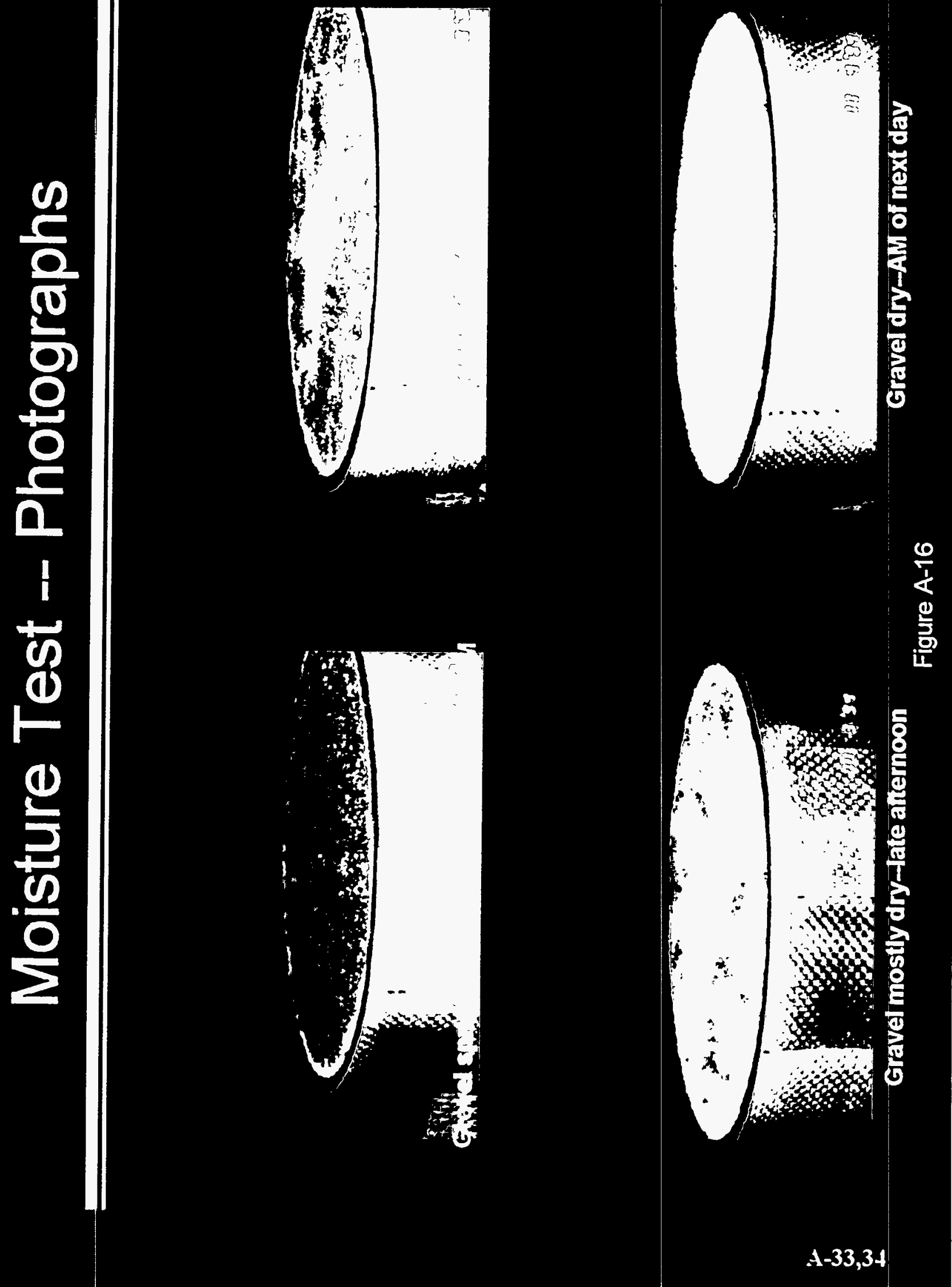


\section{Tire Tracks (Wet)}

\section{Azimuth Rotation}

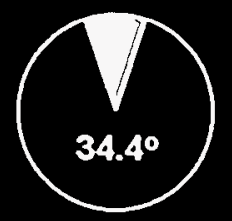

-File: CON9

- Chirp BW: $8 \mathrm{GHz}$

aCenter Freq.: $12 \mathrm{GHz}$

$\ulcorner$ Pol.:HH

$\times$ Weighting: Hamming

aY Weighting: Hamming

Image Resolution: 1.1 in.

aXY Pixel Size: 0.64 in.

- Processing Method: Subtraction
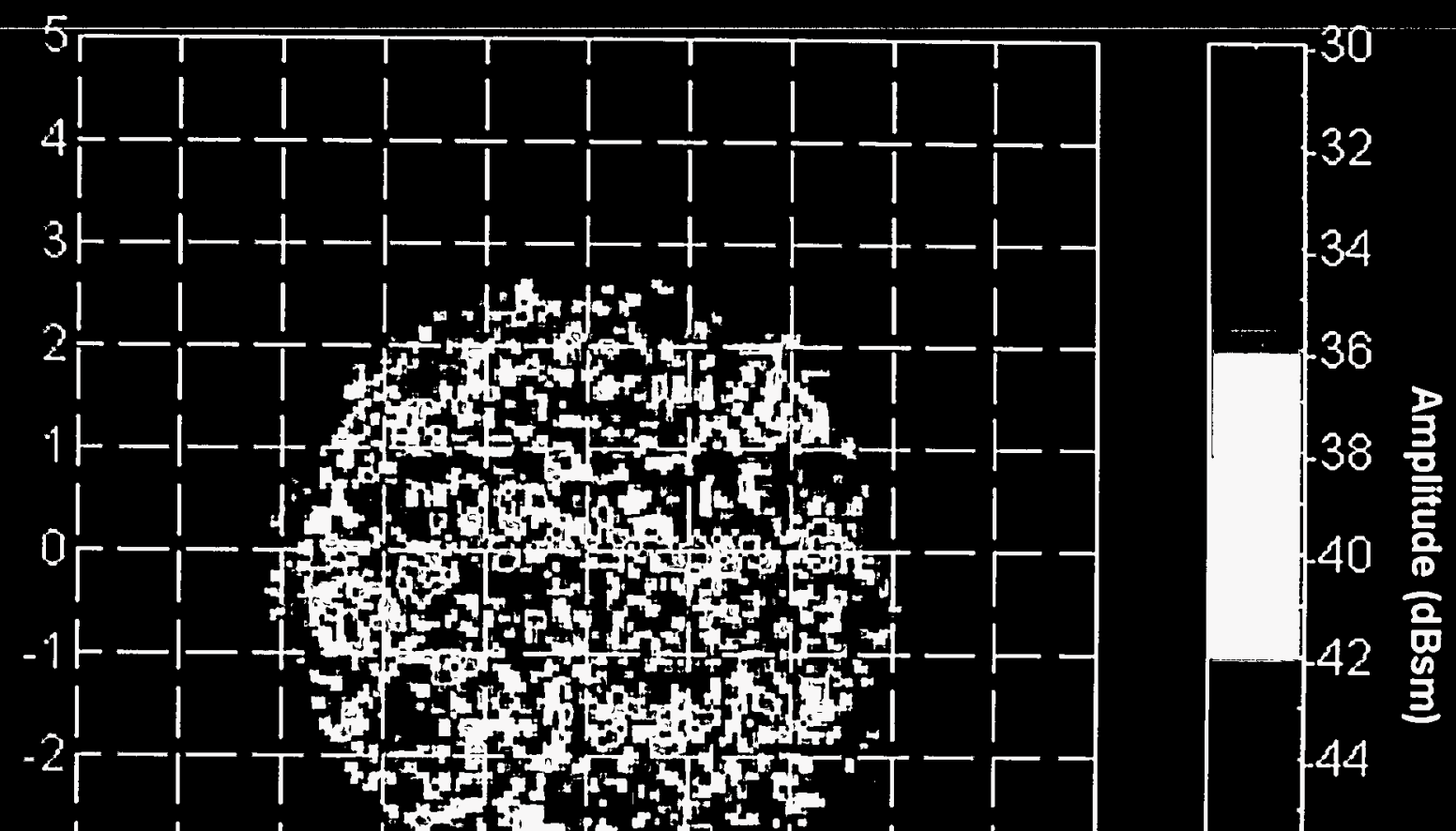

$-3 t-1-+-1+1+10-1$

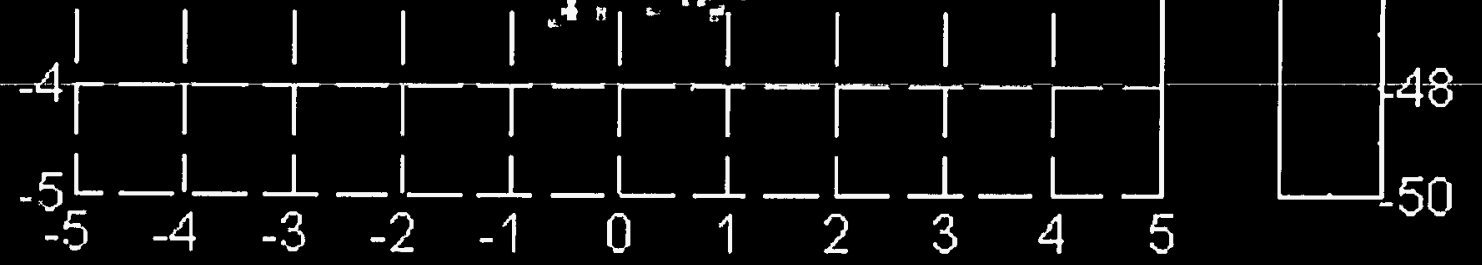

Cross-Range (it)

Figure A-17 
Amplitude (dBsm)

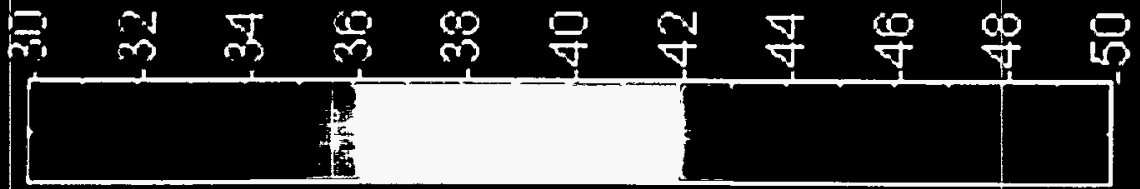

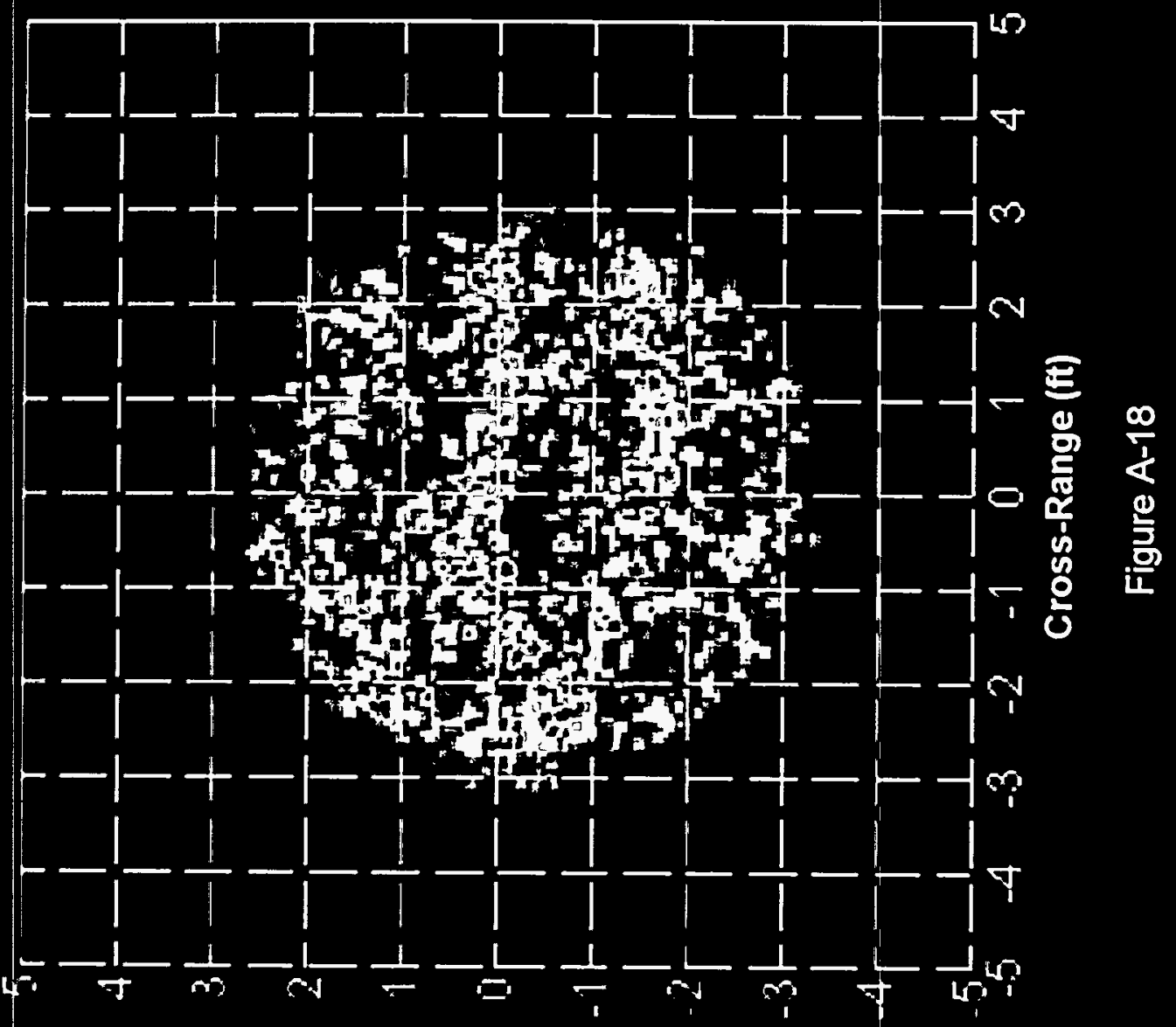

(ม) әбиey-umog
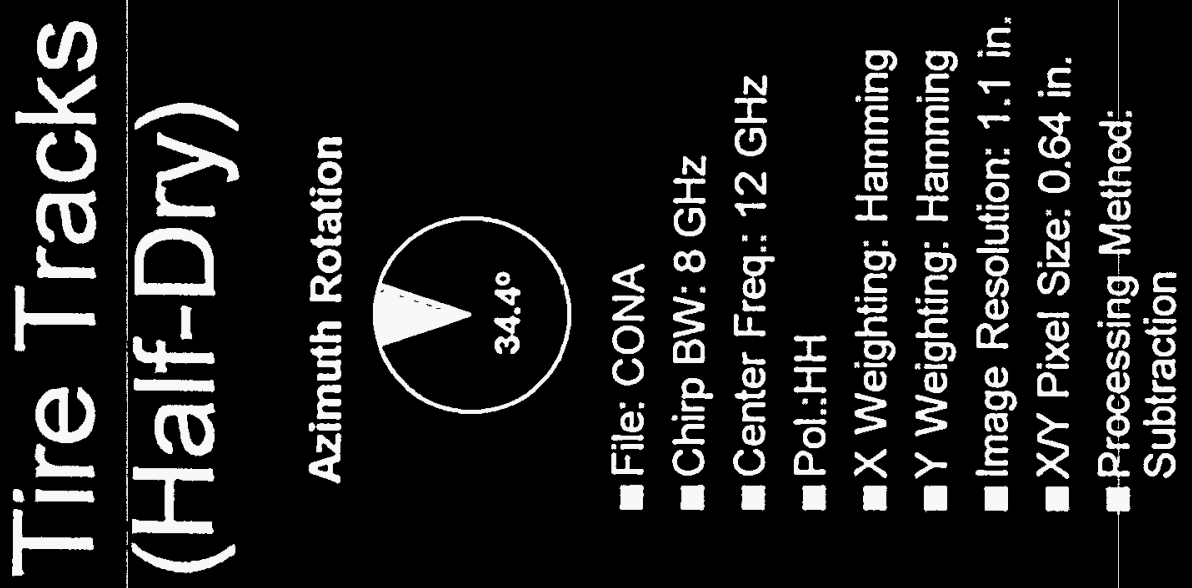


\section{Tire Tracks (Mostly-Dry)}

Azimuth Rotation

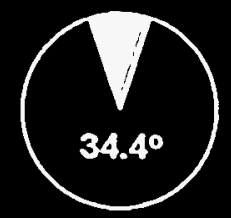

aFile: CONB

-Chirp BW: $8 \mathrm{GHz}$

aCenter Freq.: $12 \mathrm{GHz}$

$\ulcorner$ Pol.:HH

$\square \mathrm{X}$ Weighting: Hamming

-Y Weighting: Hamming

Image Resolution: 1.1 in.

aXY Pixel Size: 0.64 in.

-Processing Method:

Subtraction

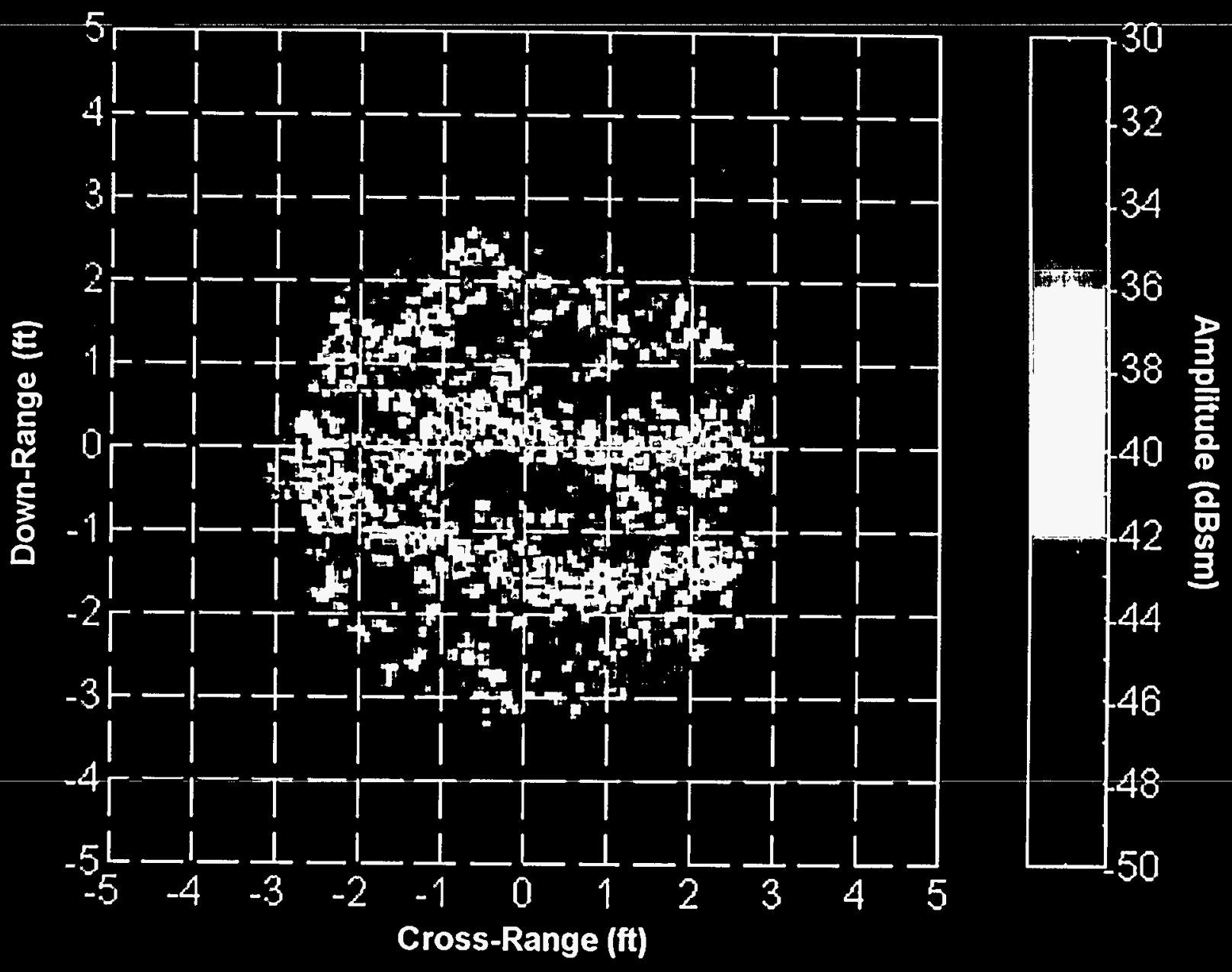

d
b

Figure A-19 


\section{Tire Tracks ("Dry")}

Azimuth Rotation

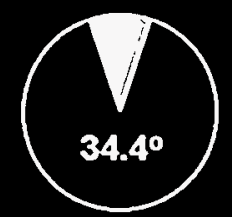

aFile: CONC

\section{aChirp BW: $8 \mathrm{GHz}$}

a Center Freq.: $12 \mathrm{GHz}$

aPol.:HH

$\square \times$ Weighting: Hamming

- Y Weighting: Hamming

Image Resolution: 1.1 in.

aXN Pixel Size: 0.64 in.

-Processing Method:

Subtraction

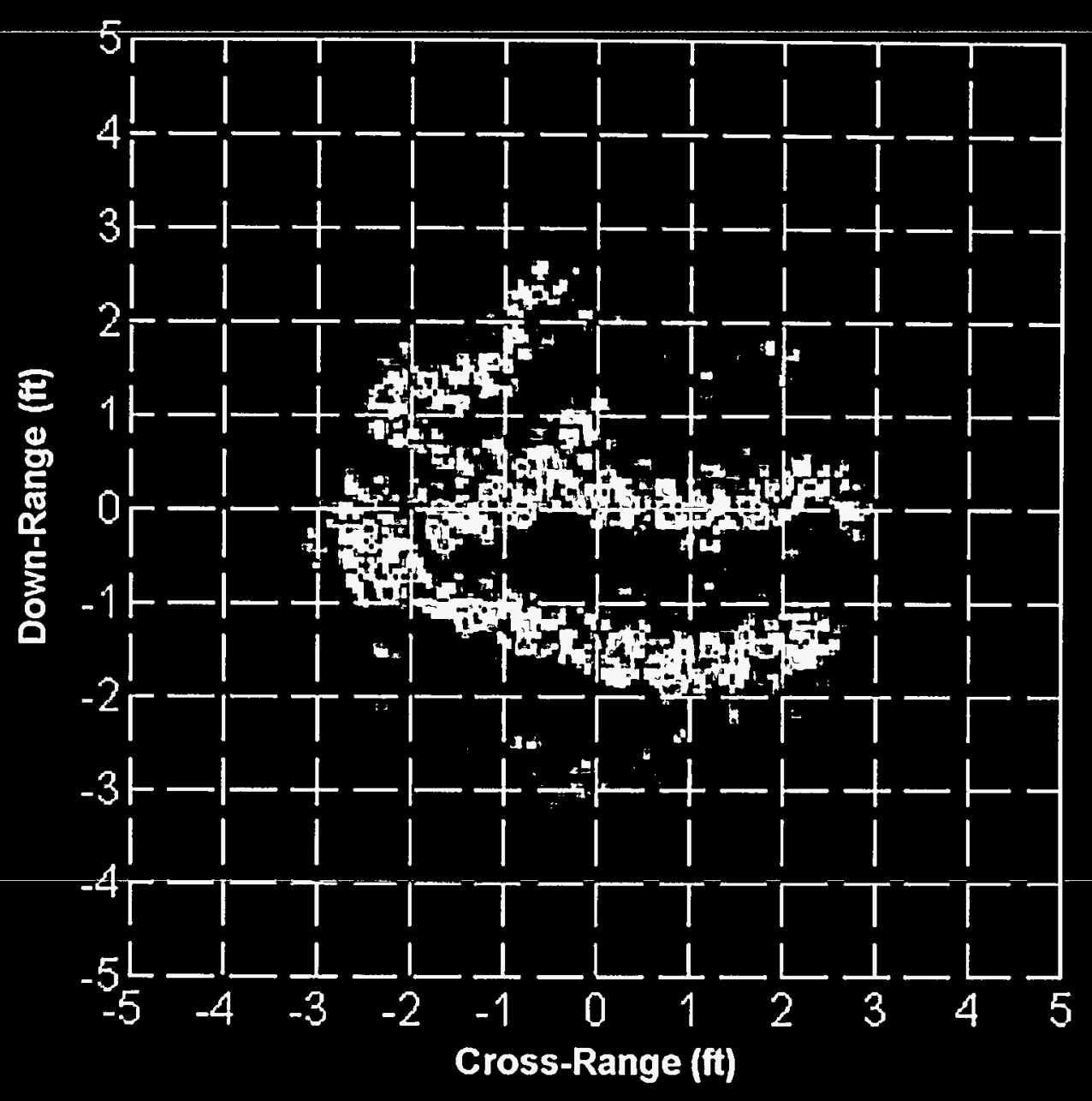

5

Figure A-20 


\section{Amplitude (dBsm)}

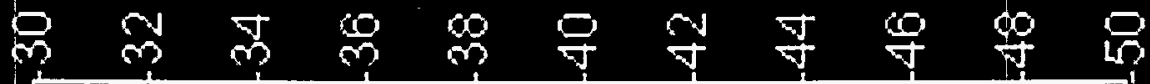

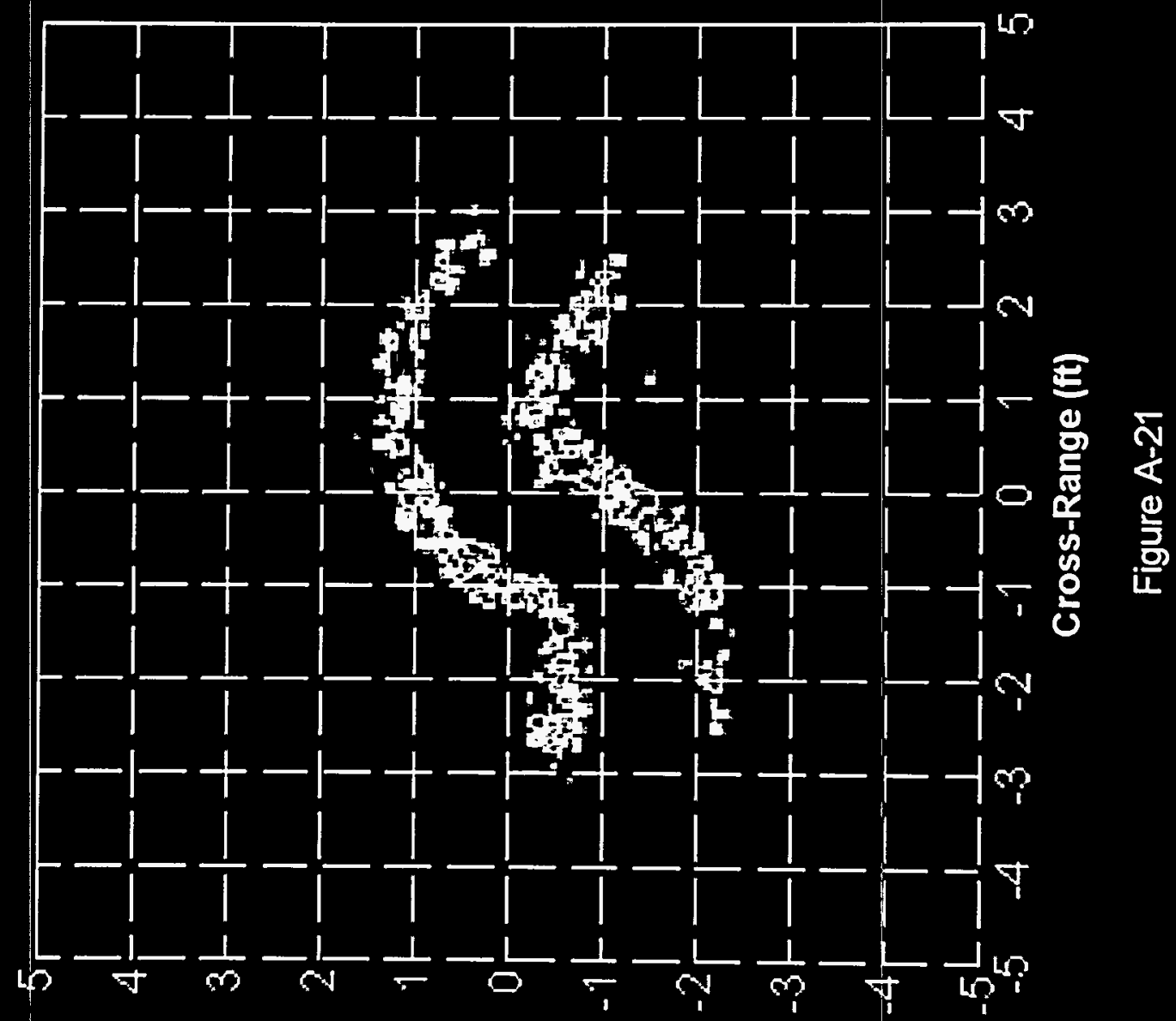

(y) әธินеม-uмо

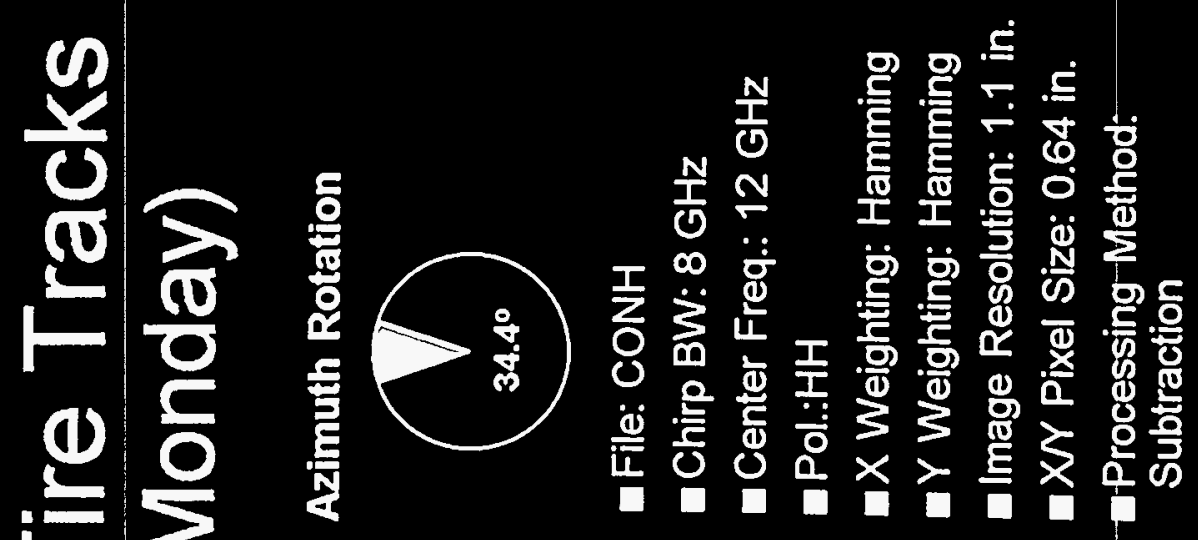




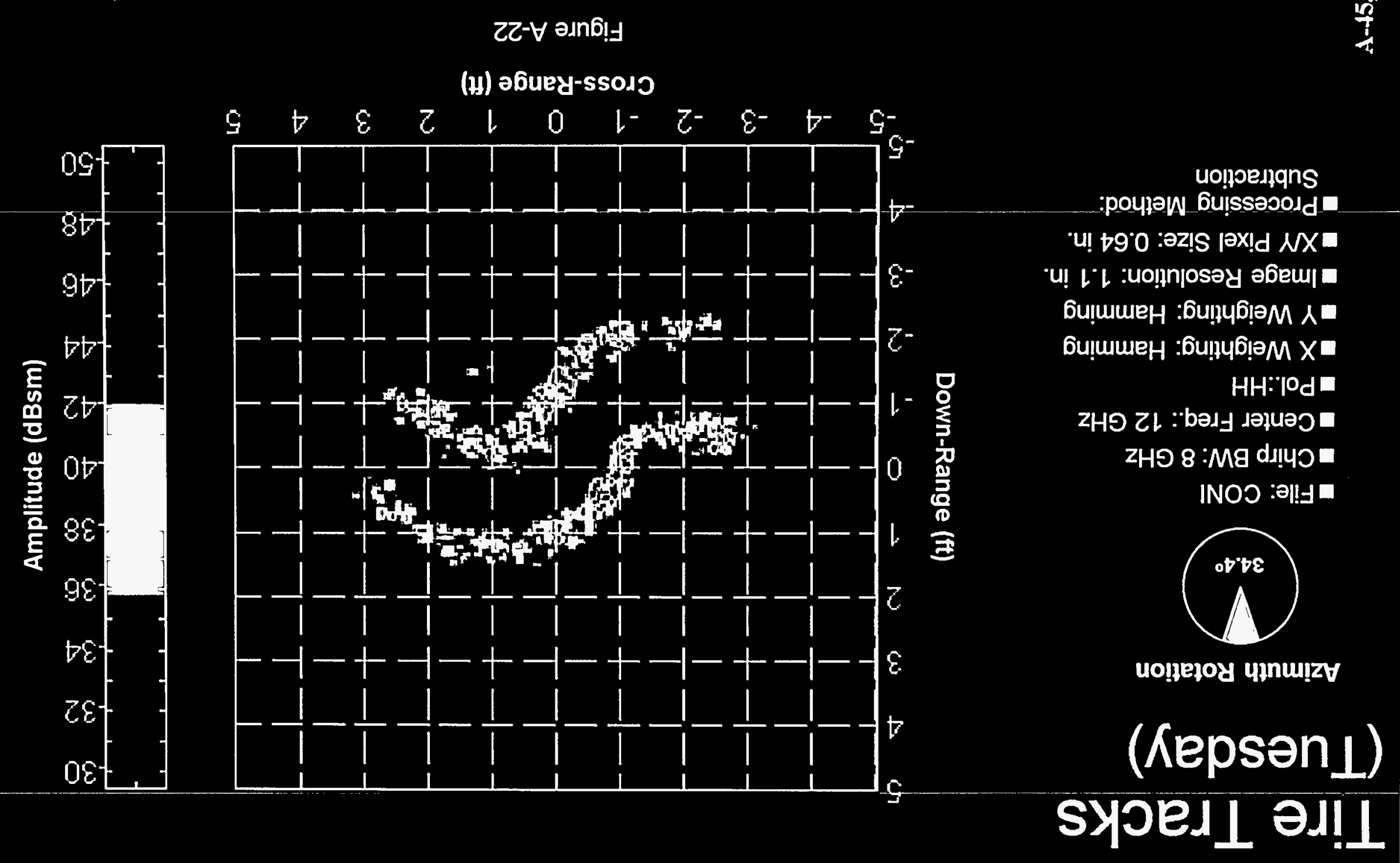




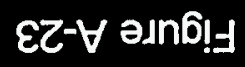

(y) əбuey-ssodo

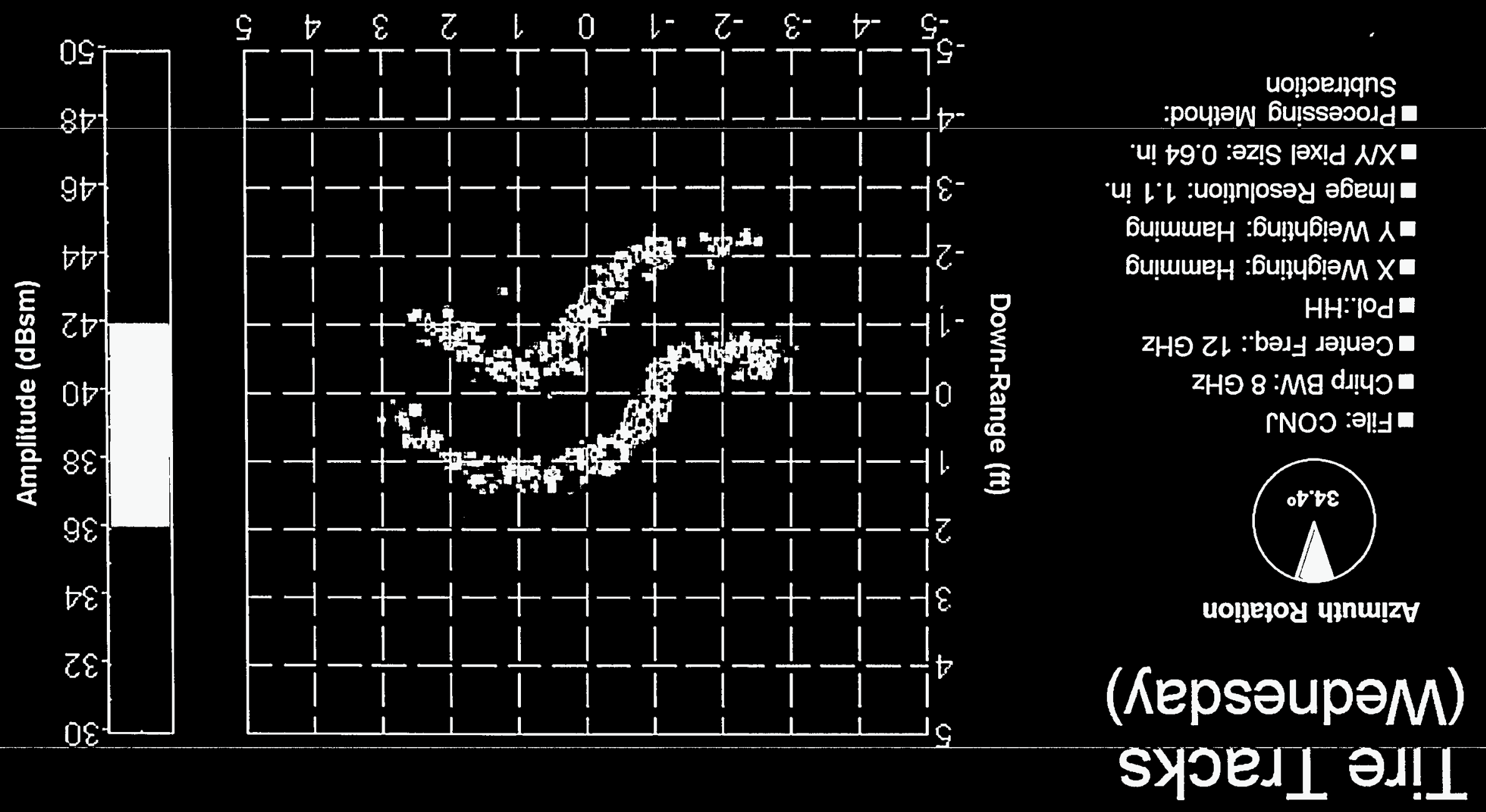




\section{Amplitude (dBsm)}

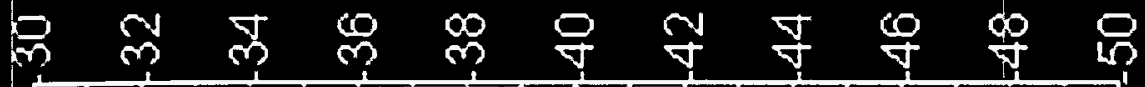

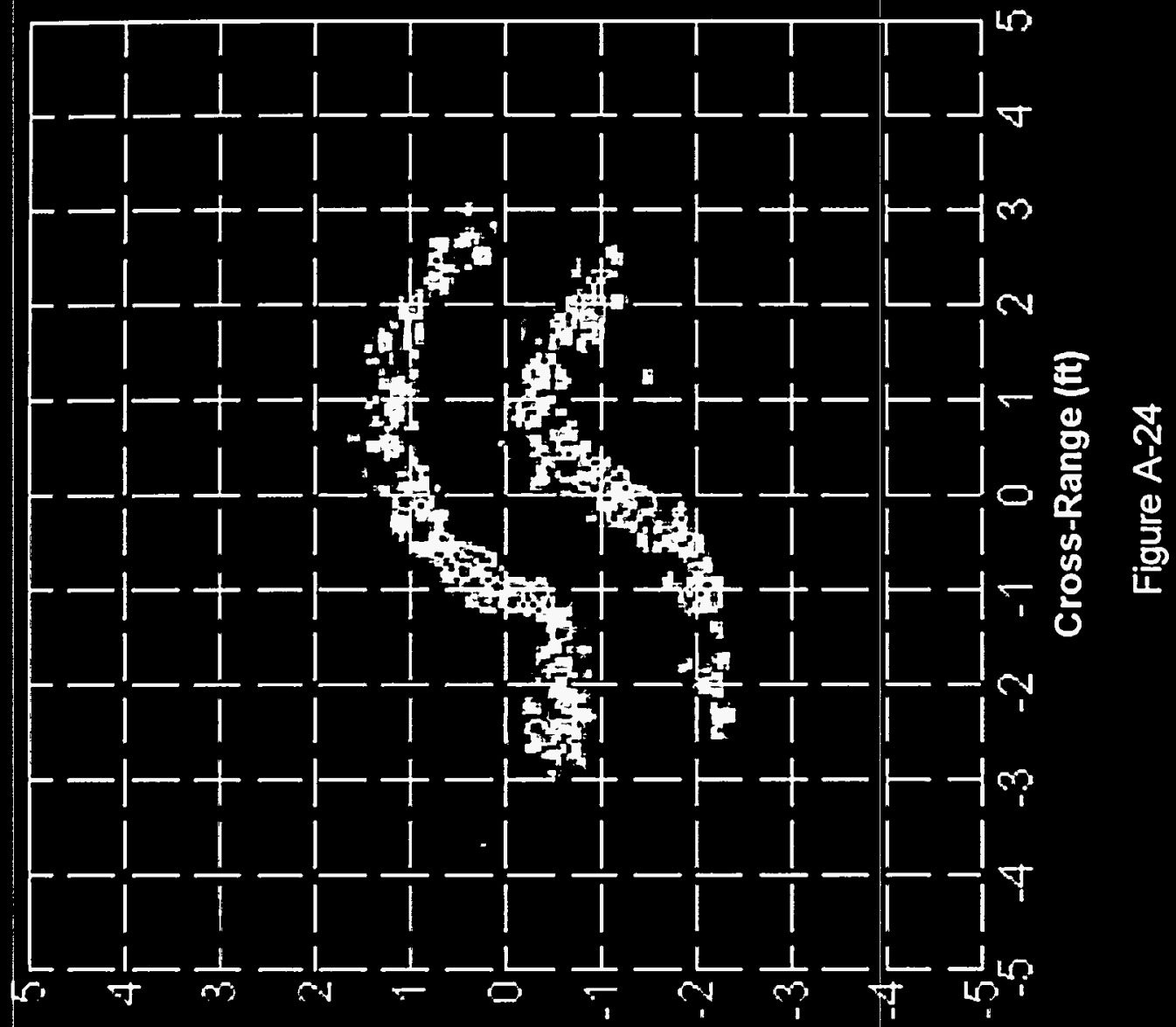

(y) อकินey-umog

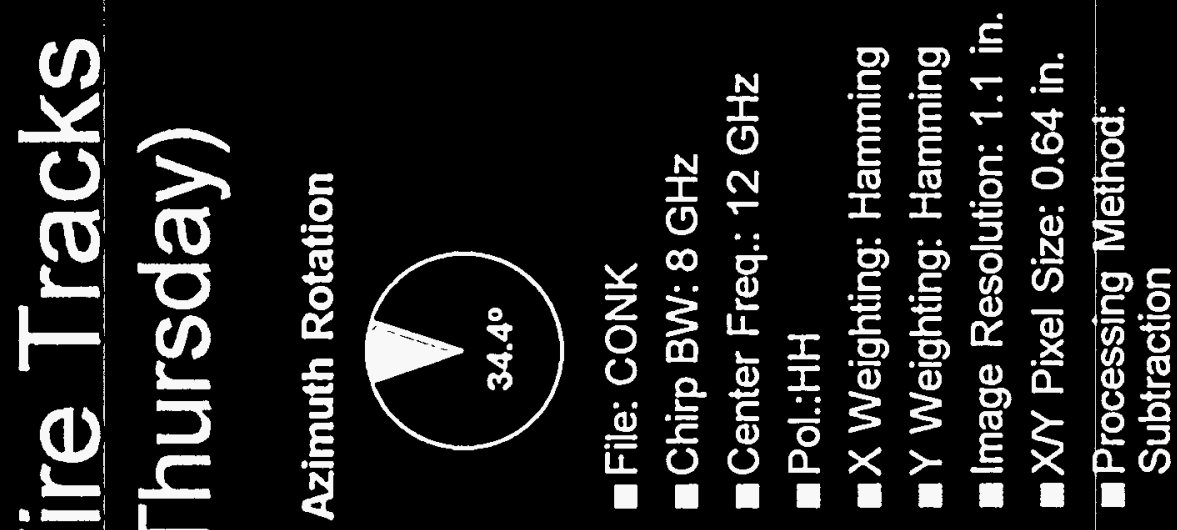




\section{Tire Tracks (Friday)}

Azimuth Rotation

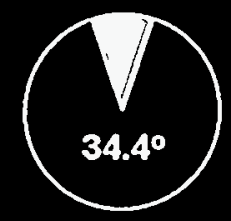

aFile: CONL

I Chirp BW: $8 \mathrm{GHz}$ uCenter Freq.: $12 \mathrm{GHz}$ Pol.:HH

aX Weighting: Hamming

Y Weighting: Hamming - Image Resolution: 1.1 in. aXY Pixel Size: 0.64 in.

Drocessing Method: Subtraction

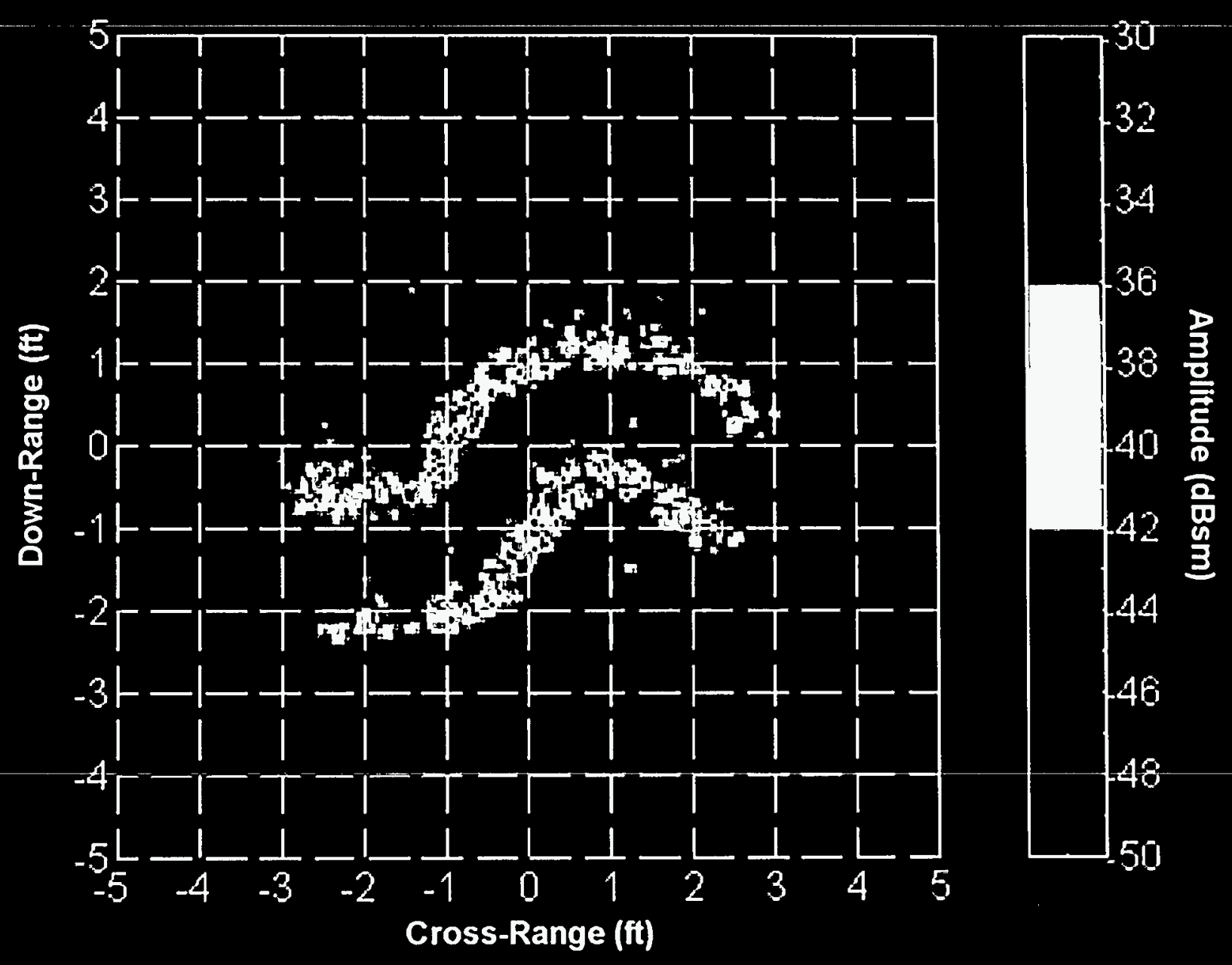

幽

Figure A-25 


\section{APPENDIX B}

CCD Images Processed Using

Maximum Likelihood Estimate of Correlation 
Intentionally Left Blank

\section{B-2}


Maximum Likelihood Estimate of Correlation -- $\gamma$

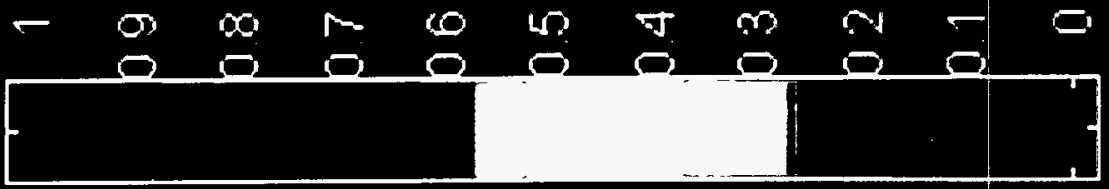

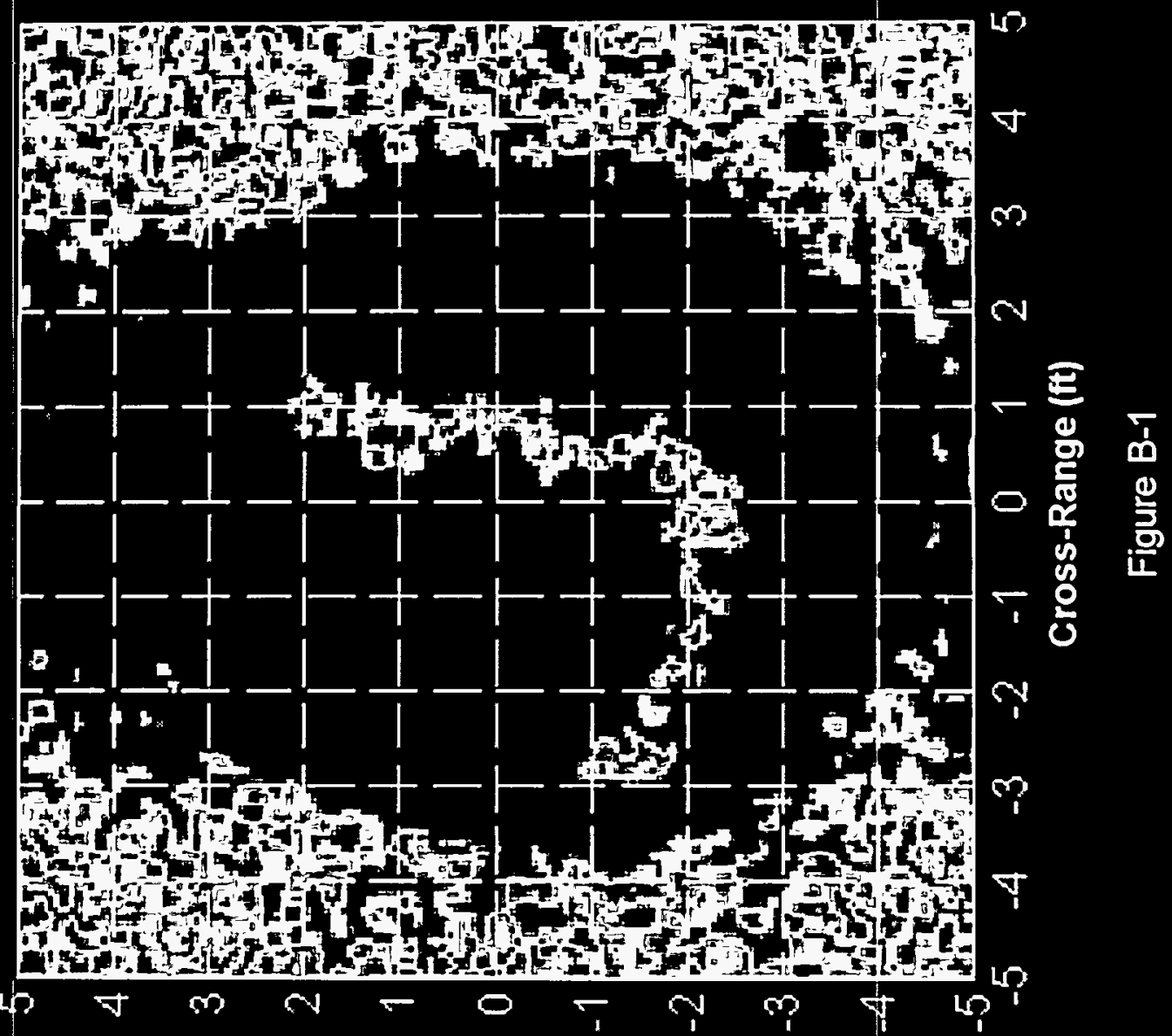

(y) จбиey-umog

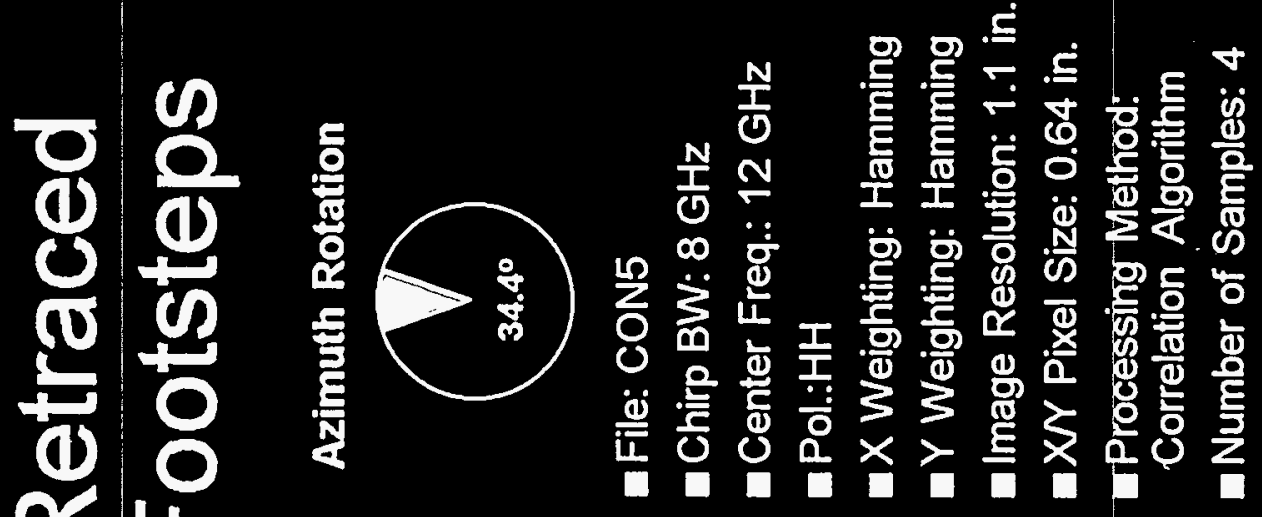




\section{Discrete Footsteps}

Azimuth Rotation

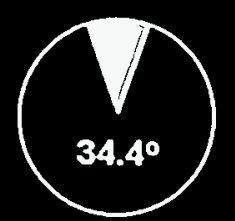

aFile: CON4

Chirp BW: $8 \mathrm{GHz}$

-Center Freq.: $12 \mathrm{GHz}$

Pol.:HH

$X$ Weighting: Hamming $Y$ Weighting: Hamming Image Resolution: 1.1 in. -XY Pixel Size: 0.64 in.

aProcessing Method: Correlation Algorithm

number of Samples: 5
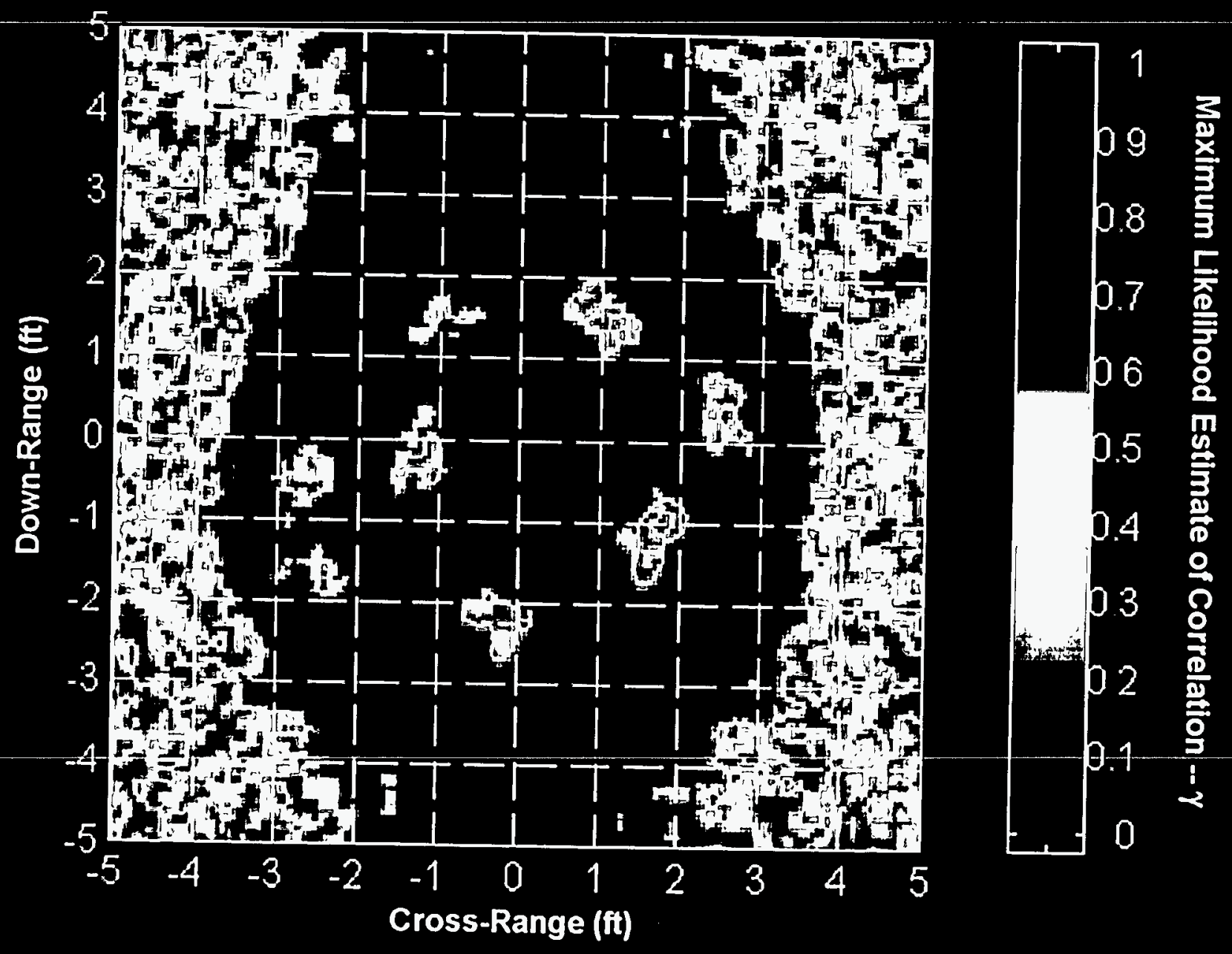

$\ddot{\alpha}$
$a$

Figure B-2 


\section{Discrete Footsteps}

\section{Azimuth Rotation}

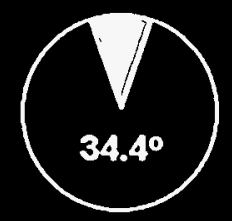

aFile: CON4

$\ulcorner$ Chirp BW: 8 GHz

$\ulcorner$ Center Freq.: $12 \mathrm{GHz}$

口Pol.:W

$\ulcorner\mathrm{X}$ Weighting: Hamming

aY Weighting: Hamming

- Image Resolution: 1.1 in.

aXV Pixel Size: 0.64 in.

-Processing-Method: Correlation Algorithm

- Number of Samples: 5

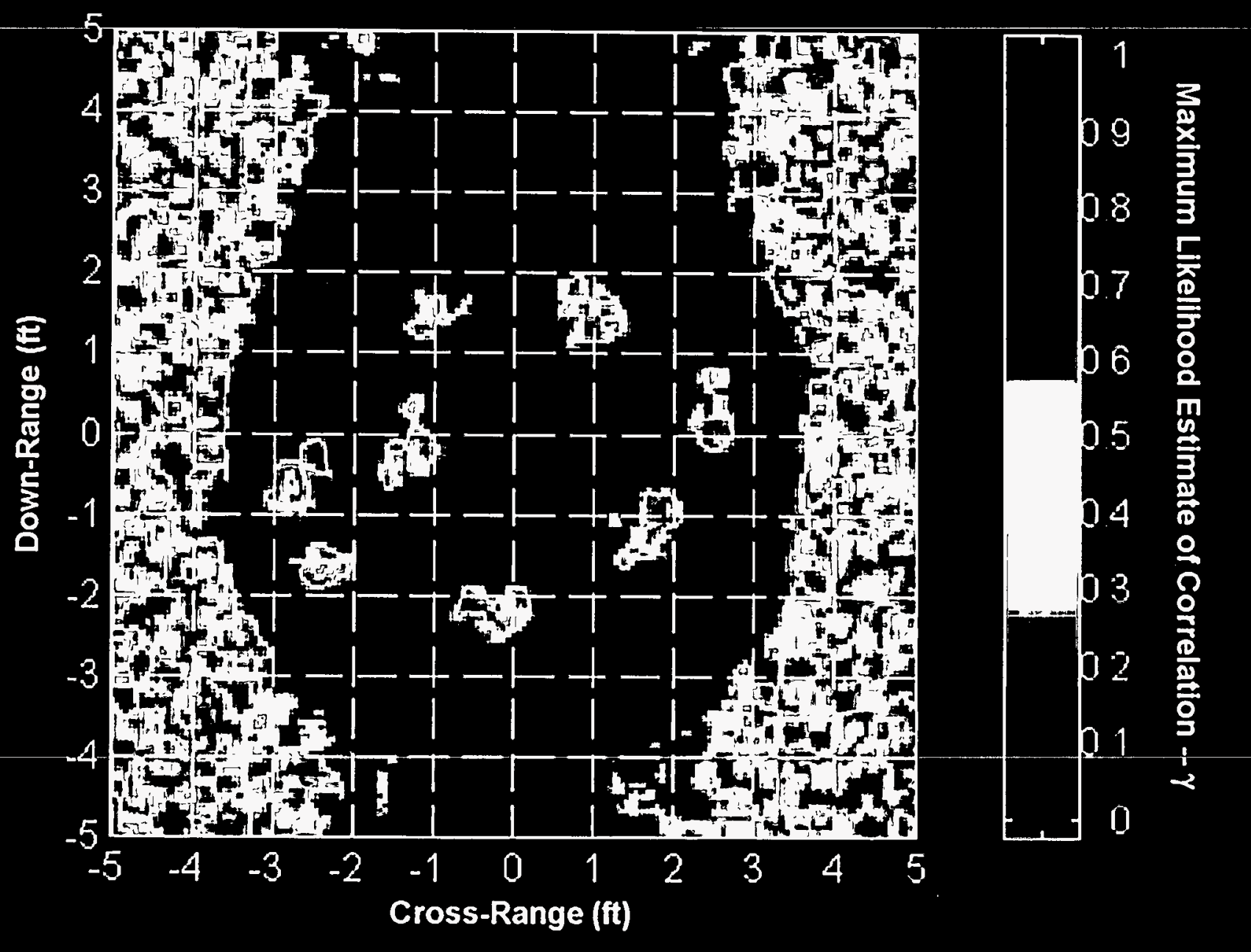

Figure B-3

$\underset{\substack{0 \\ \infty}}{\infty}$ 
Maximum Likelihood Estimate of Correlation $-\boldsymbol{\gamma}$
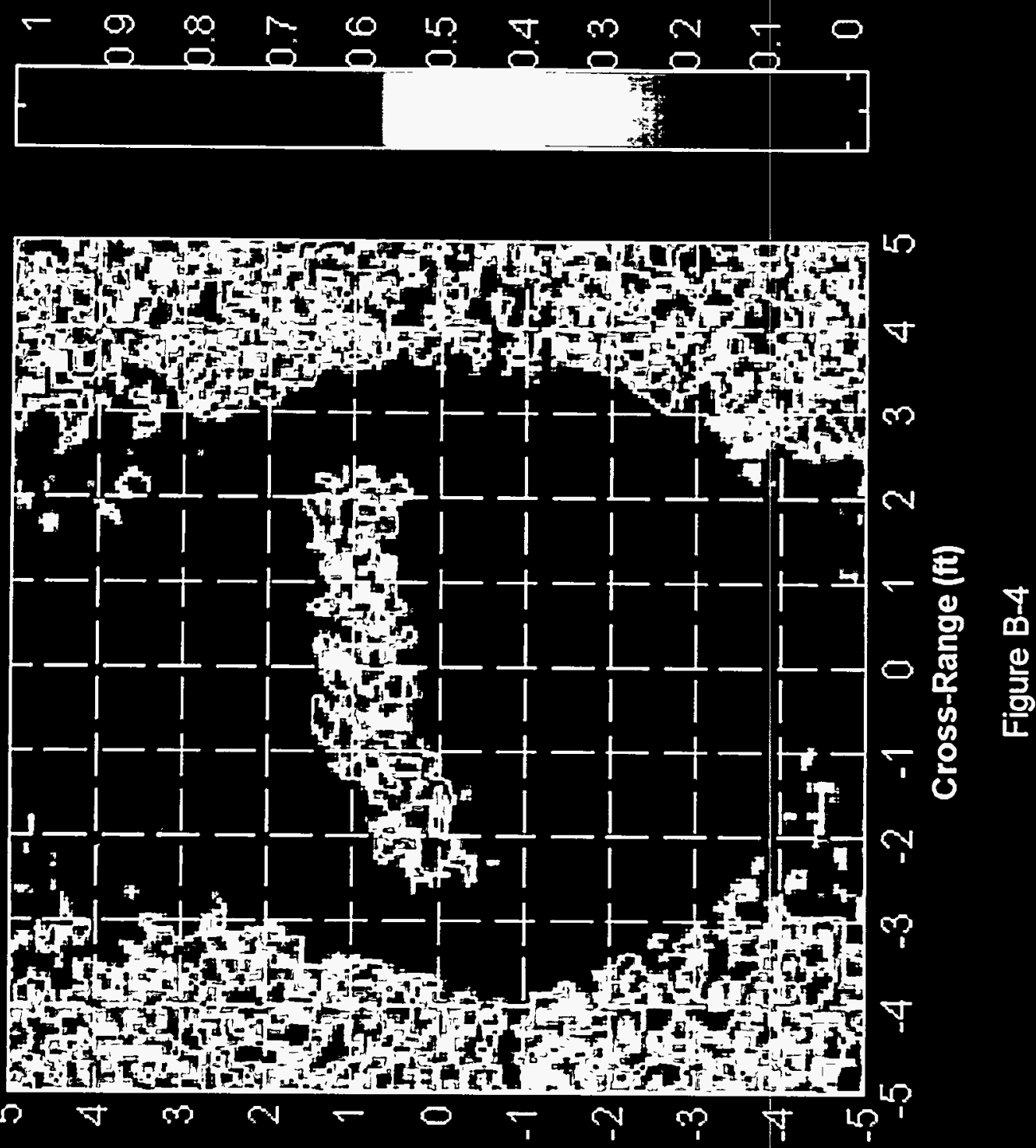

(y) จธินеy-uмо0

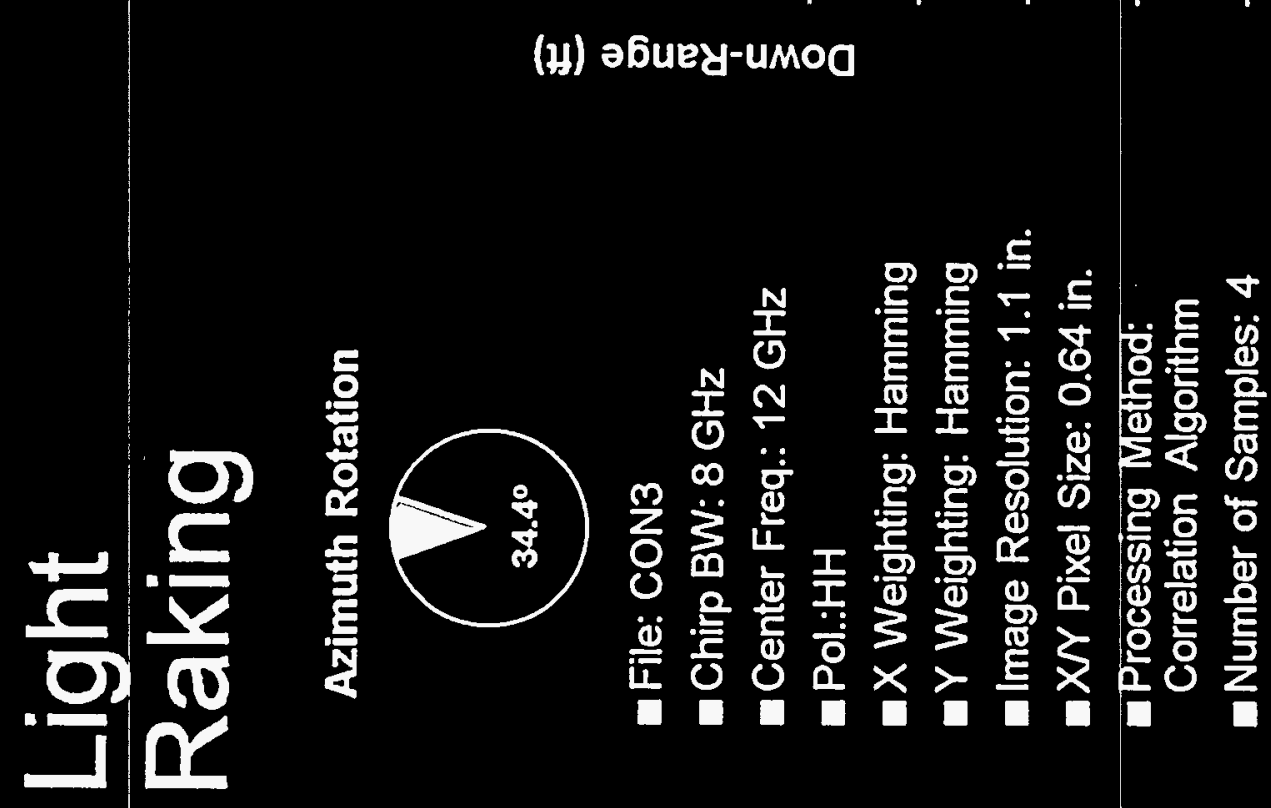


Maximum Likelihood Estimate of Correlation - $\gamma$

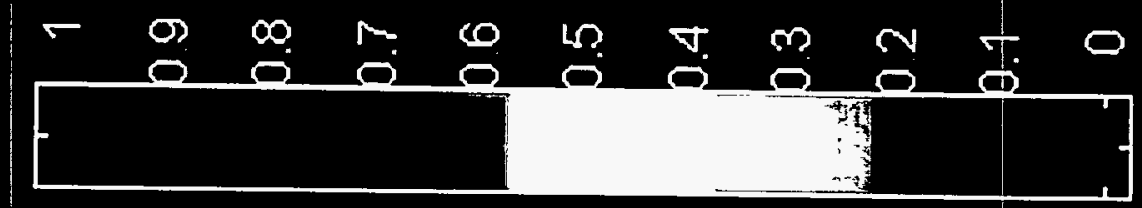

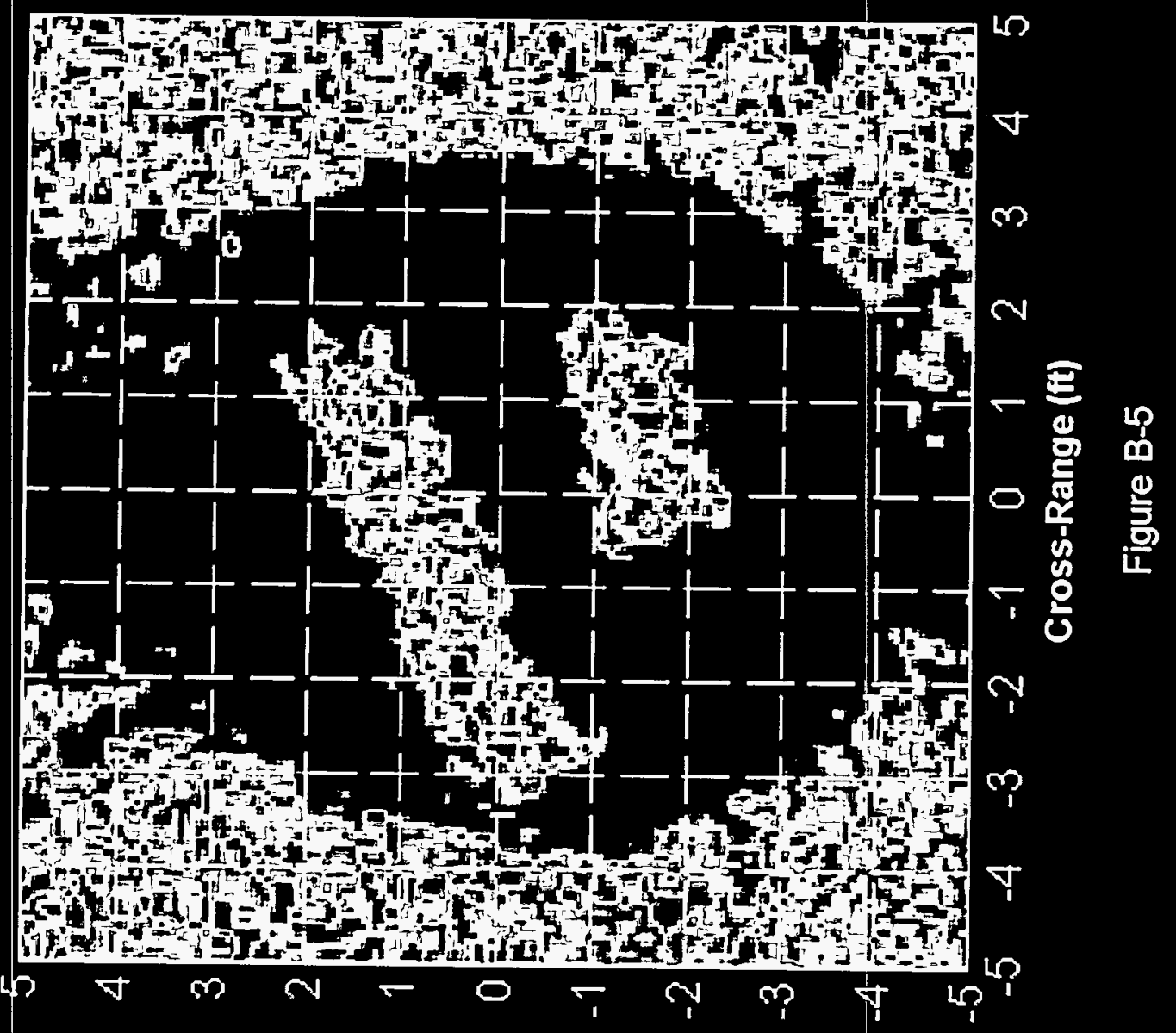

(y) әธิuey-uмо

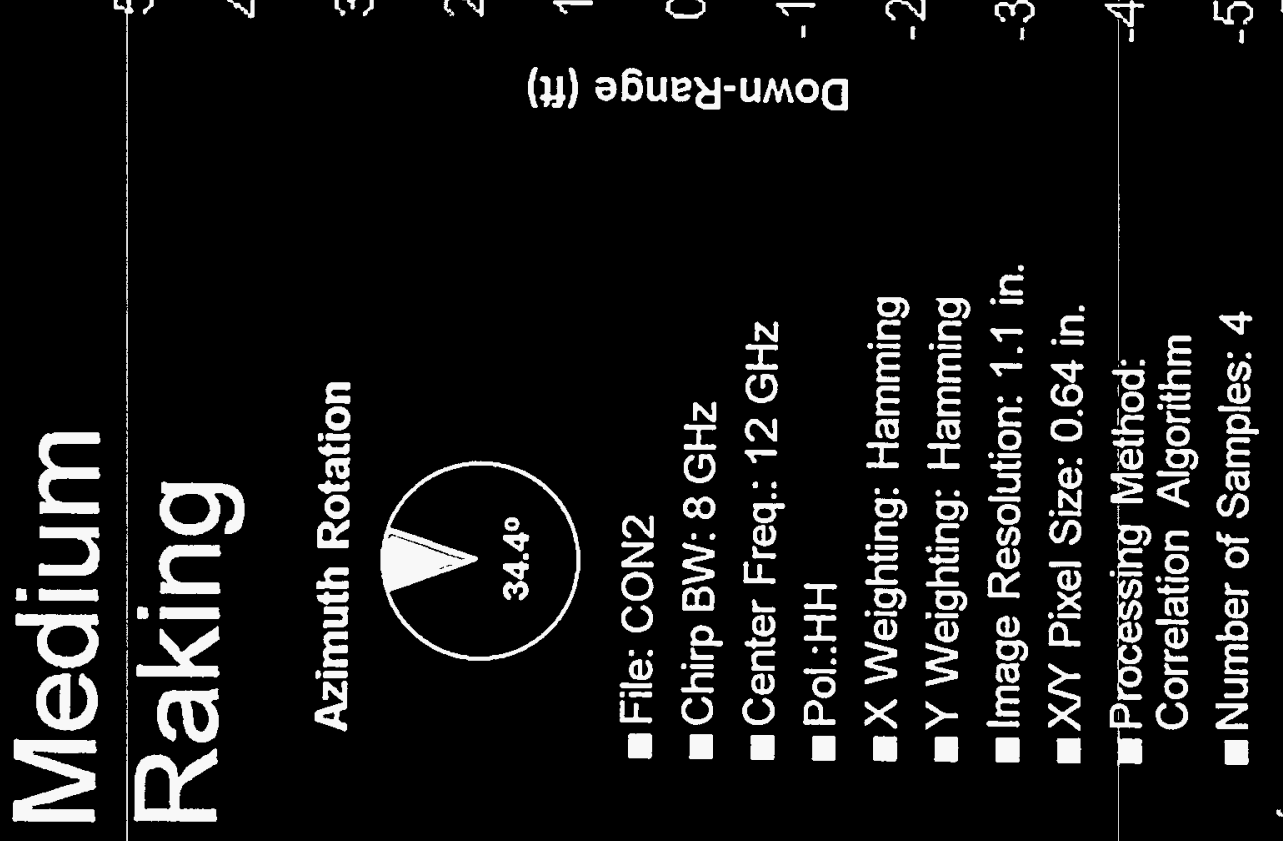


Maximum Likelihood Estimate of Correlation $-\gamma$

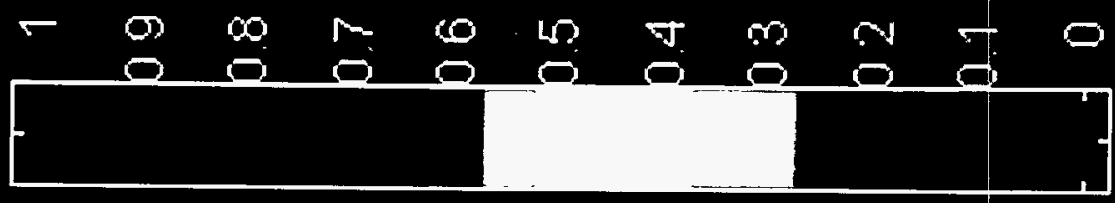

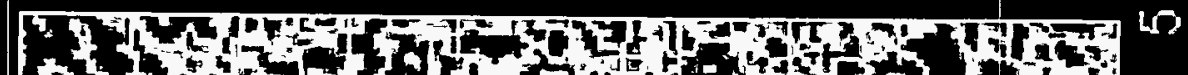

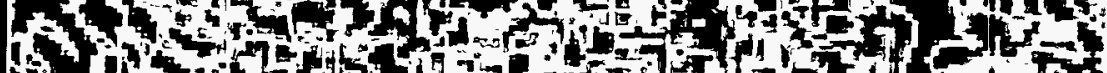

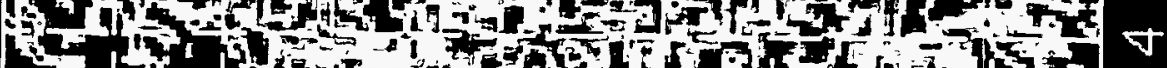

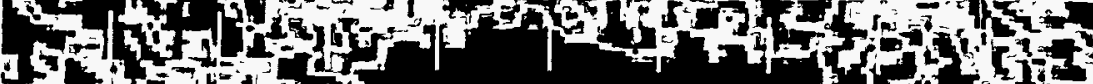

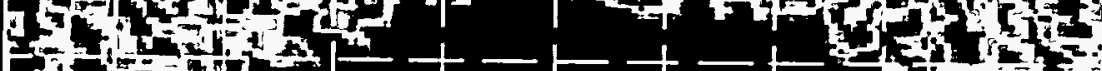

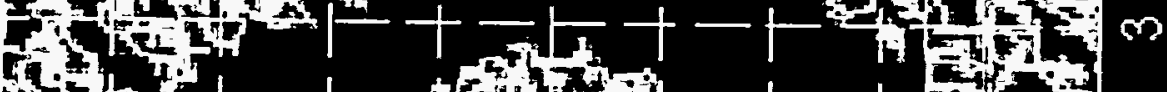

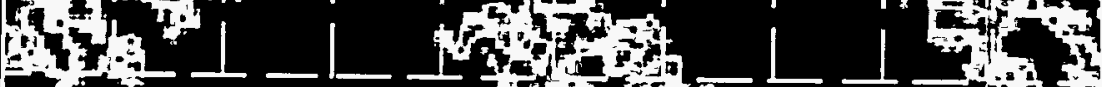

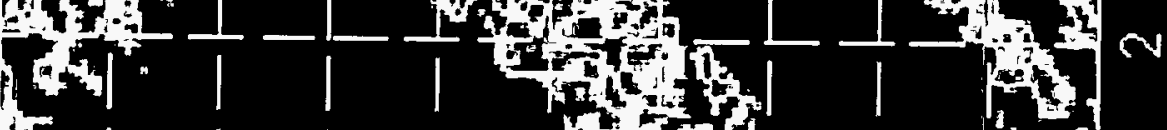

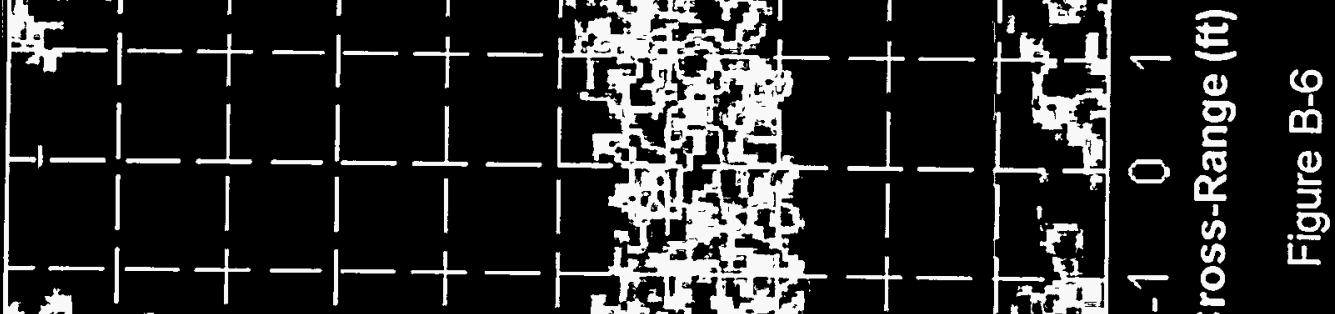

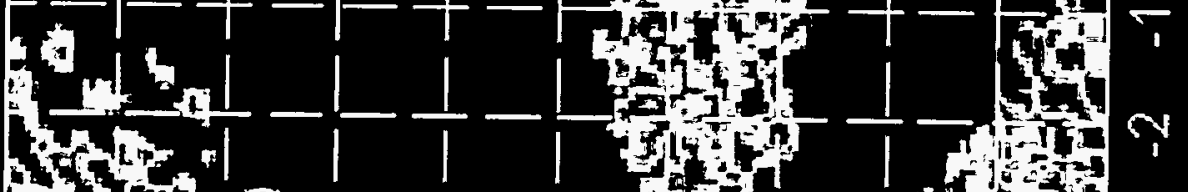

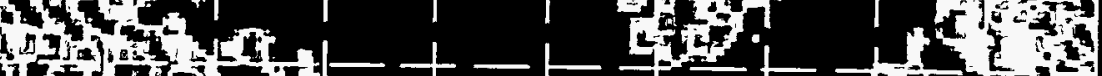

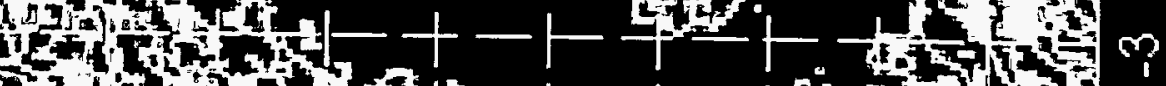

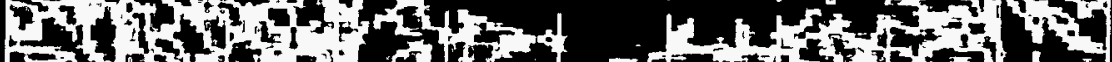

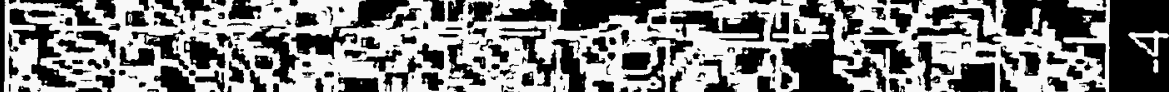

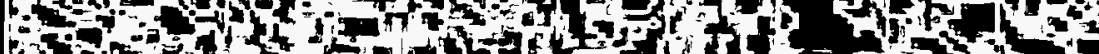

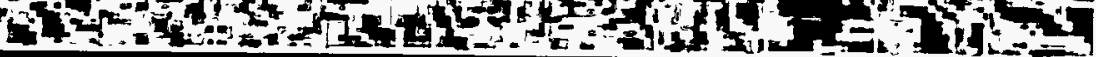

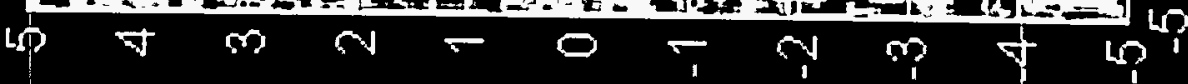

(y) จธินey-umog

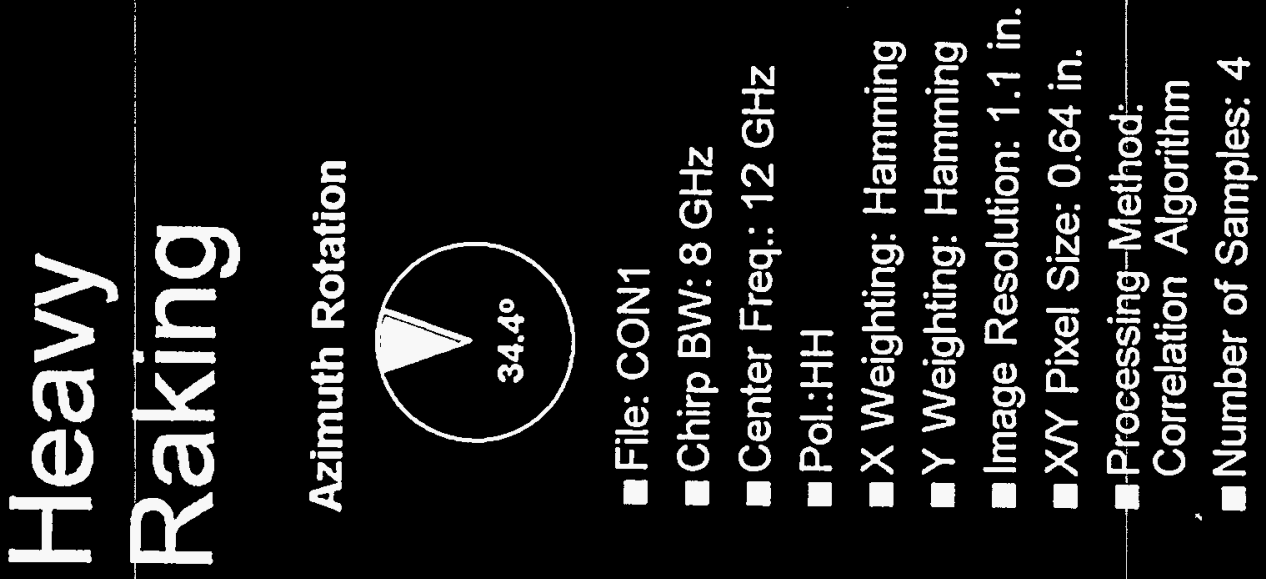




\section{Discrete Rock Displacement}

Azimuth Rotation

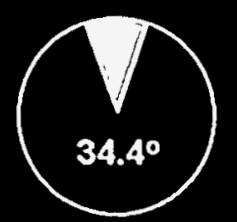

afile: CON6

aChirp BW: $8 \mathrm{GHz}$

uCenter Freq.: $12 \mathrm{GHz}$

aPol.:HH

$\square X$ Weighting: Hamming

aY Weighting: Hamming ulmage Resolution: 1.1 in.

aXN Pixel Size: 0.64 in.

-Processing Method:

Correlation Algorithm

- Number of Samples: 4

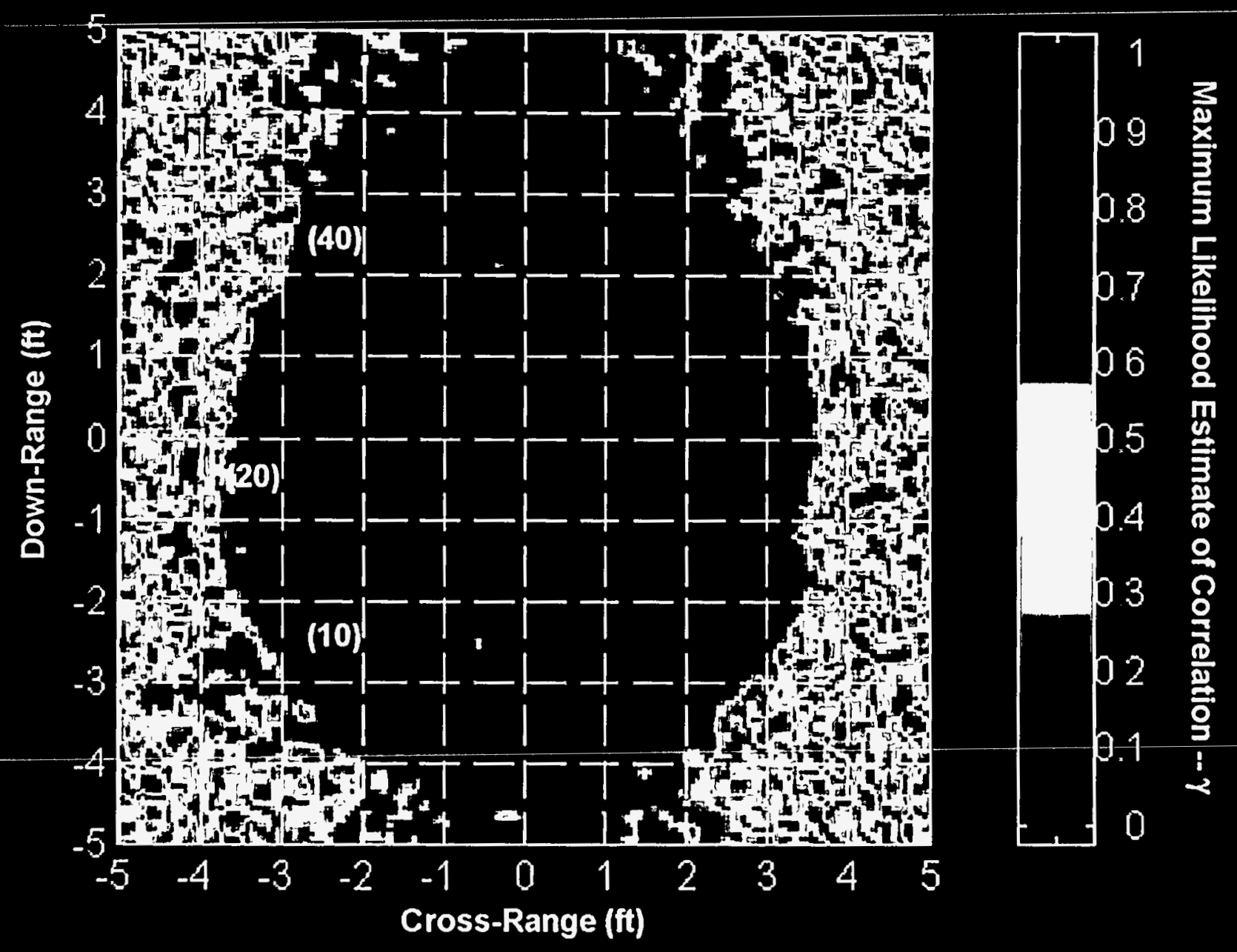

00
0
0
0

Figure B-7 
Maximum Likelihood Estimate of Correlation $-\gamma$
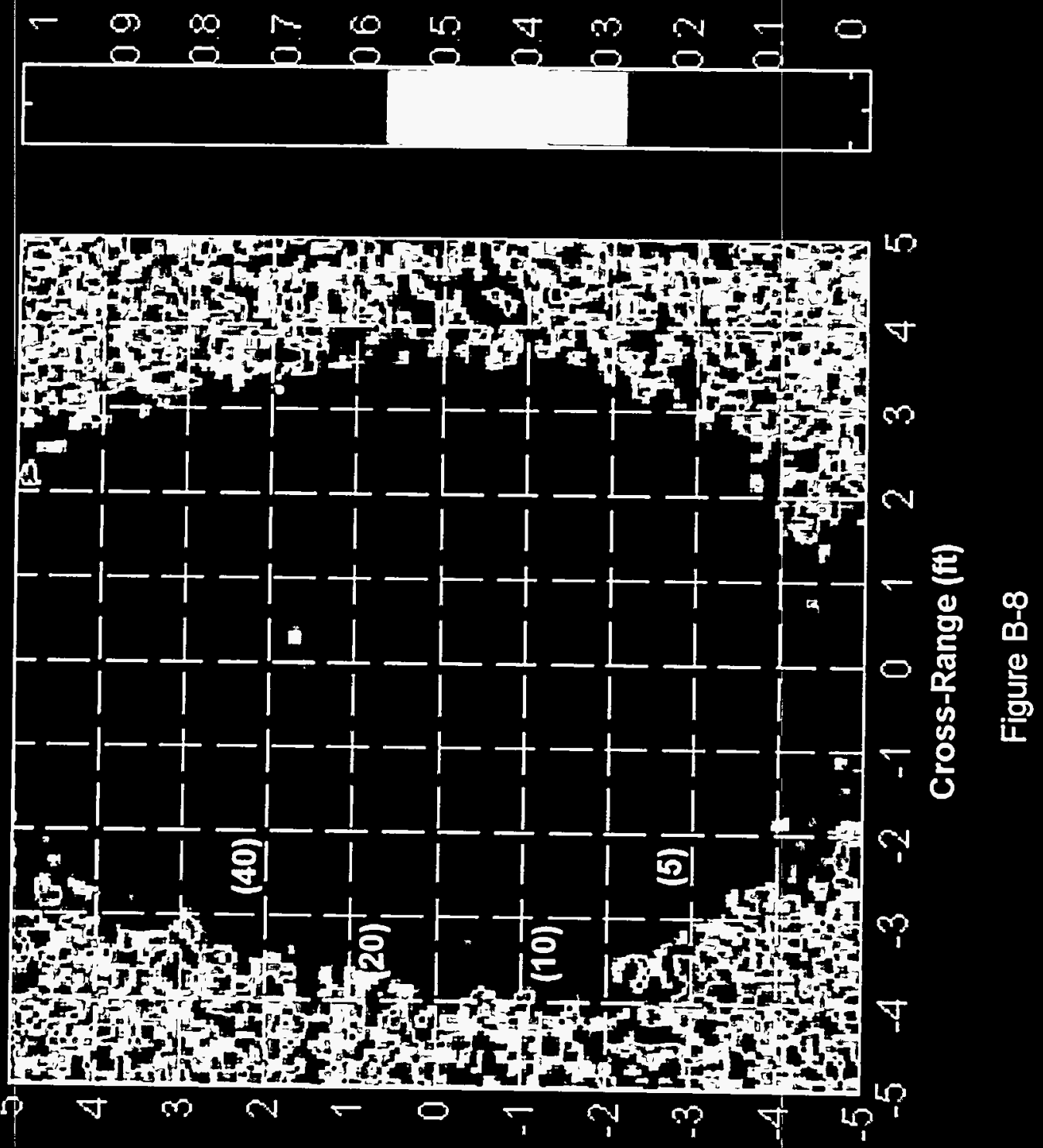

(y) әธินеy-uмоa

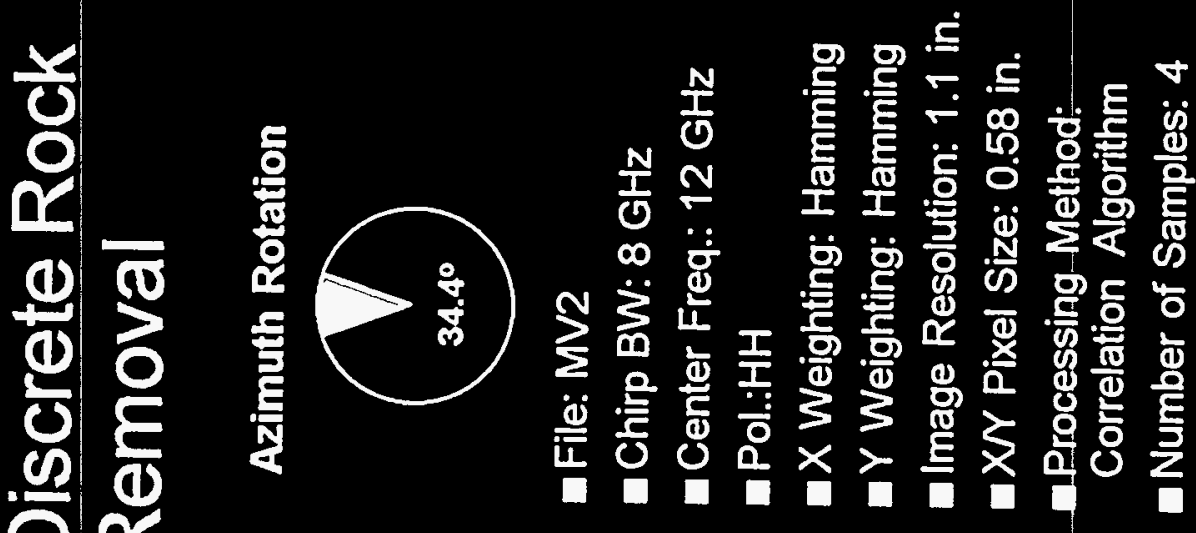




\section{Discrete Footsteps}

Azimuth Rotation

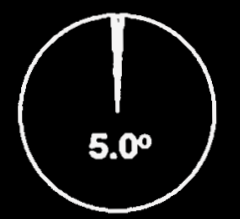

File: S1SN

-Chirp BW: $1 \mathrm{GHz}$

Center Freq.: $11.5 \mathrm{GHz}$

Pol.:HH

$X$ Weighting: Hamming

-Y Weighting: Hamming

Image Resolution: 8.7 in.

aXY Pixel Size: 3.81 in.

uProcessing Method:

Correlation Algorithm

- Number of Samples: 3

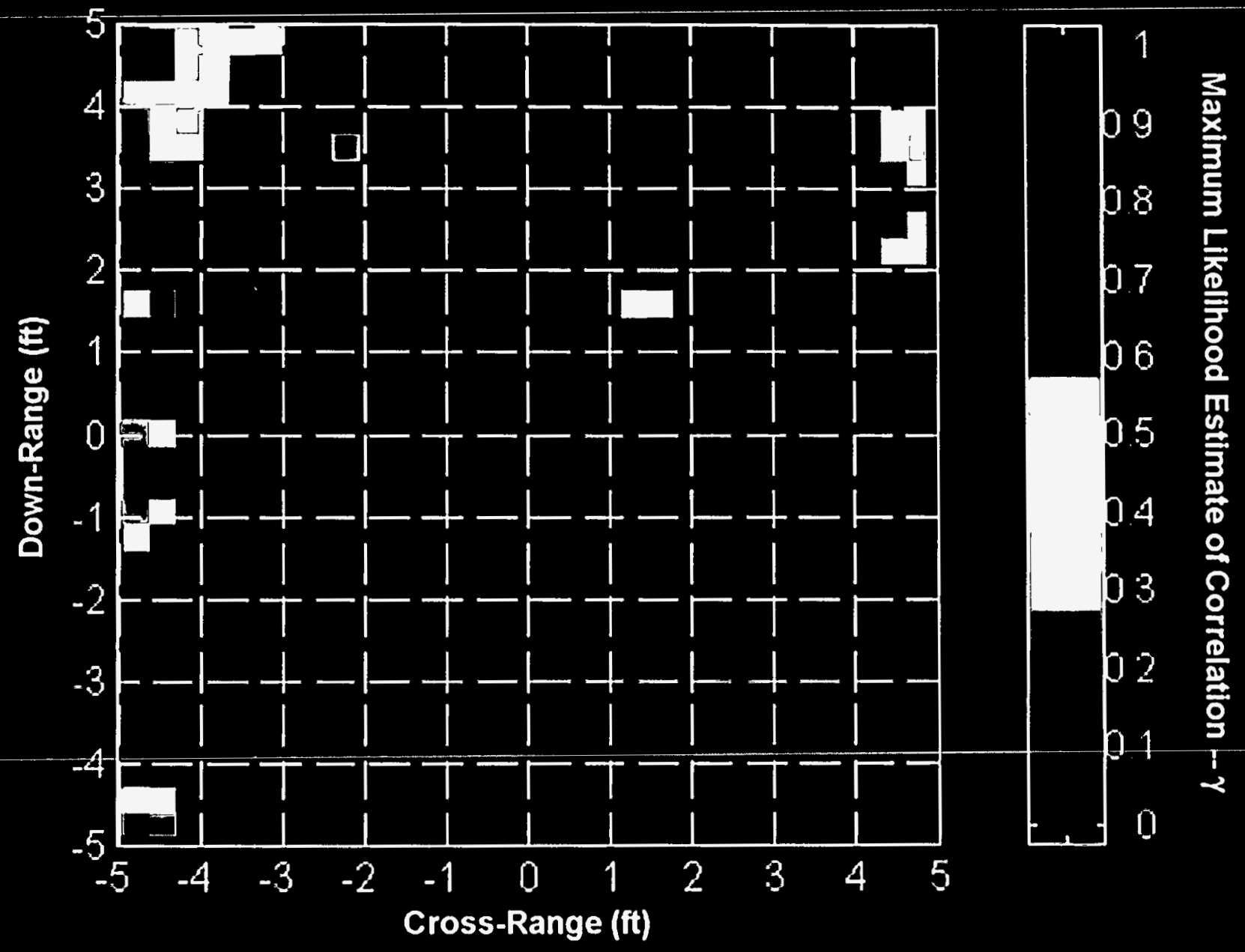

军

Figure B-9 


\section{Discrete Footsteps}

Azimuth Rotation

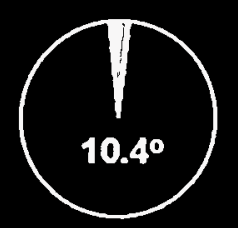

aFile: S2SN

Chirp BW: $2 \mathrm{GHz}$

Center Freq.: $11.0 \mathrm{GHz}$

Pol.:HH

$X$ Weighting: Hamming

口Y Weighting: Hamming

almage Resolution: 4.3 in.

aXY Pixel Size: 2.34 in.

uProcessing Method:

Correlation Algorithm

- Number of Samples: 3

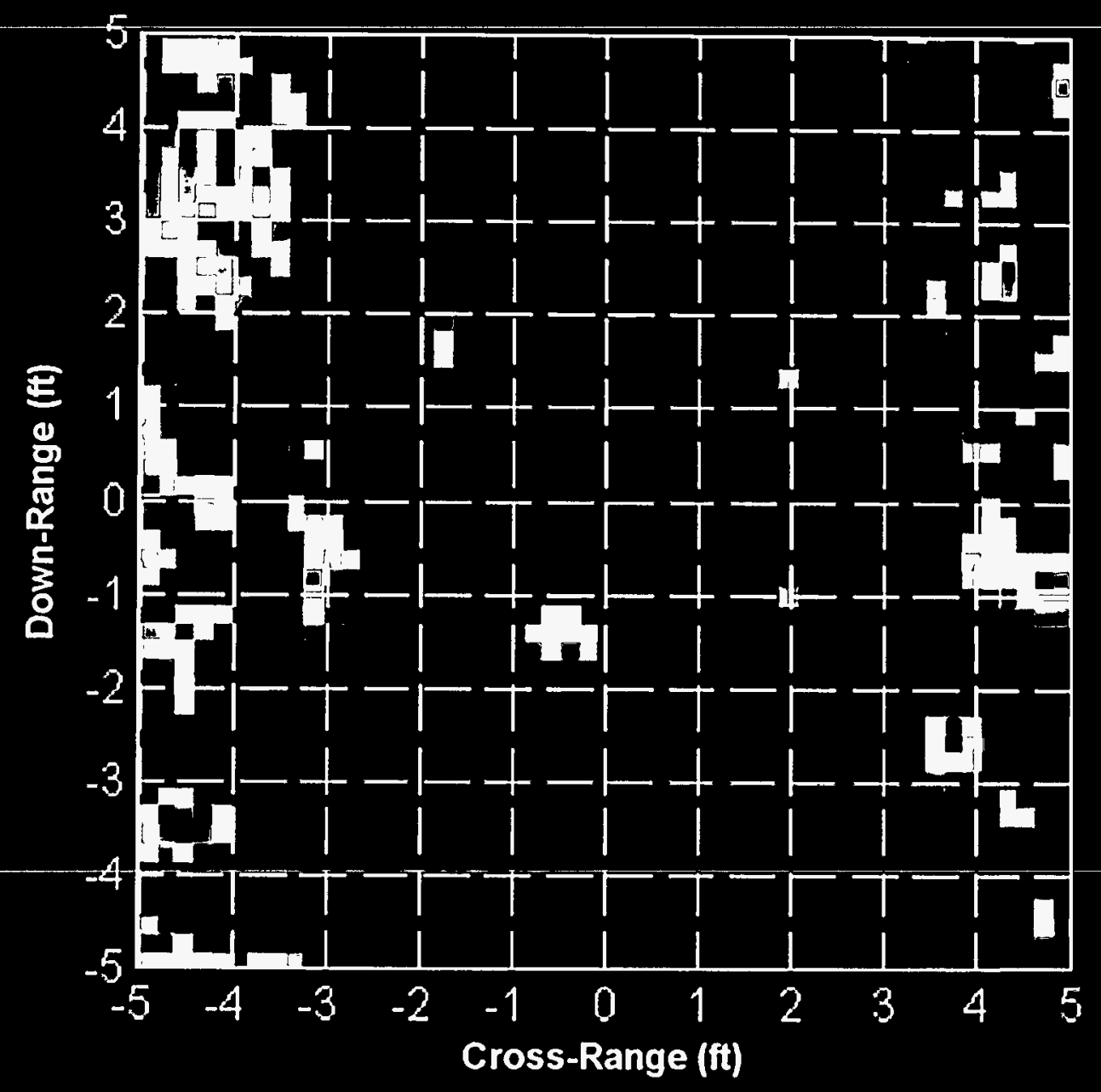

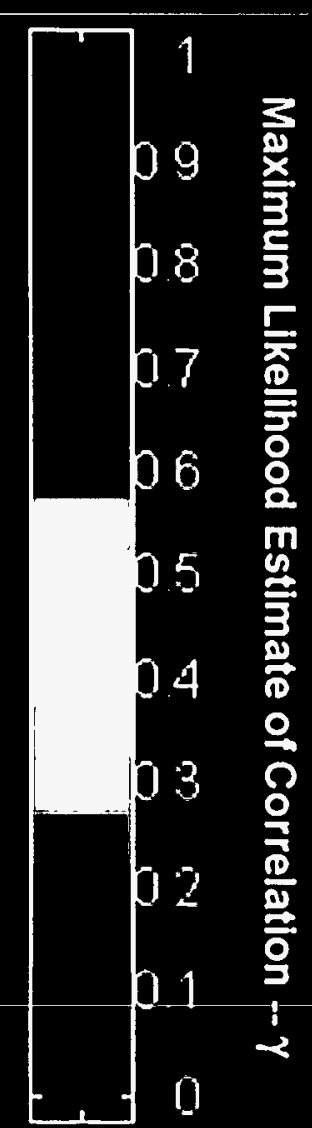

$\underset{N}{\mathbb{N}}$

Figure B-10 


\section{Discrete Footsteps}

\section{Azimuth Rotation}

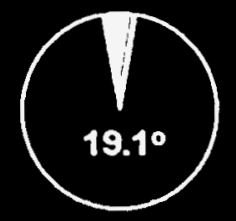

aFile: S4SN

I Chirp BW: $4 \mathrm{GHz}$

a Center Freq.: $12.0 \mathrm{GHz}$ Pol.:HH

$\times$ Weighting: Hamming

-Y Weighting: Hamming I Image Resolution: 2.2 in.

aXY Pixel Size: 1.16 in.

Processing Method:

Correlation Algorithm

n Number of Samples: 3

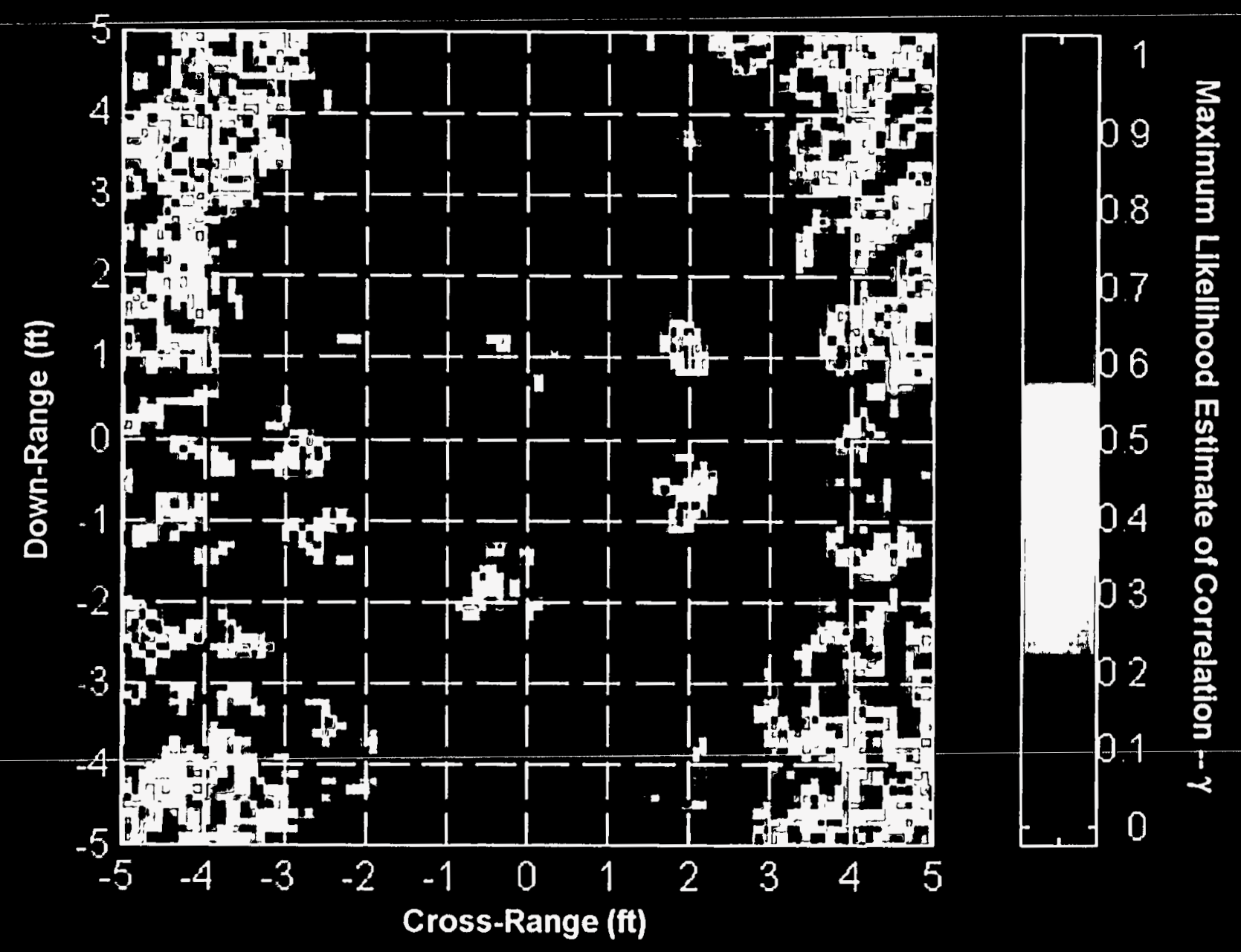

莣

Figure B-11 
Maximum Likelihood Estimate of Correlation $-\gamma$

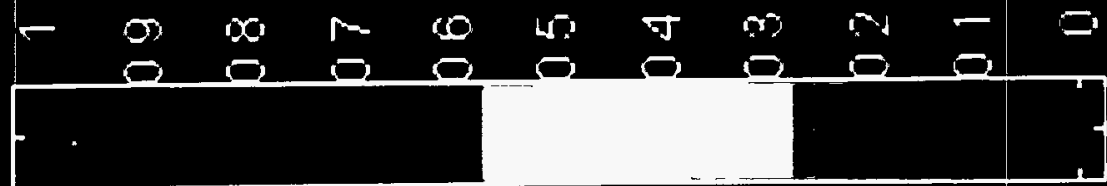

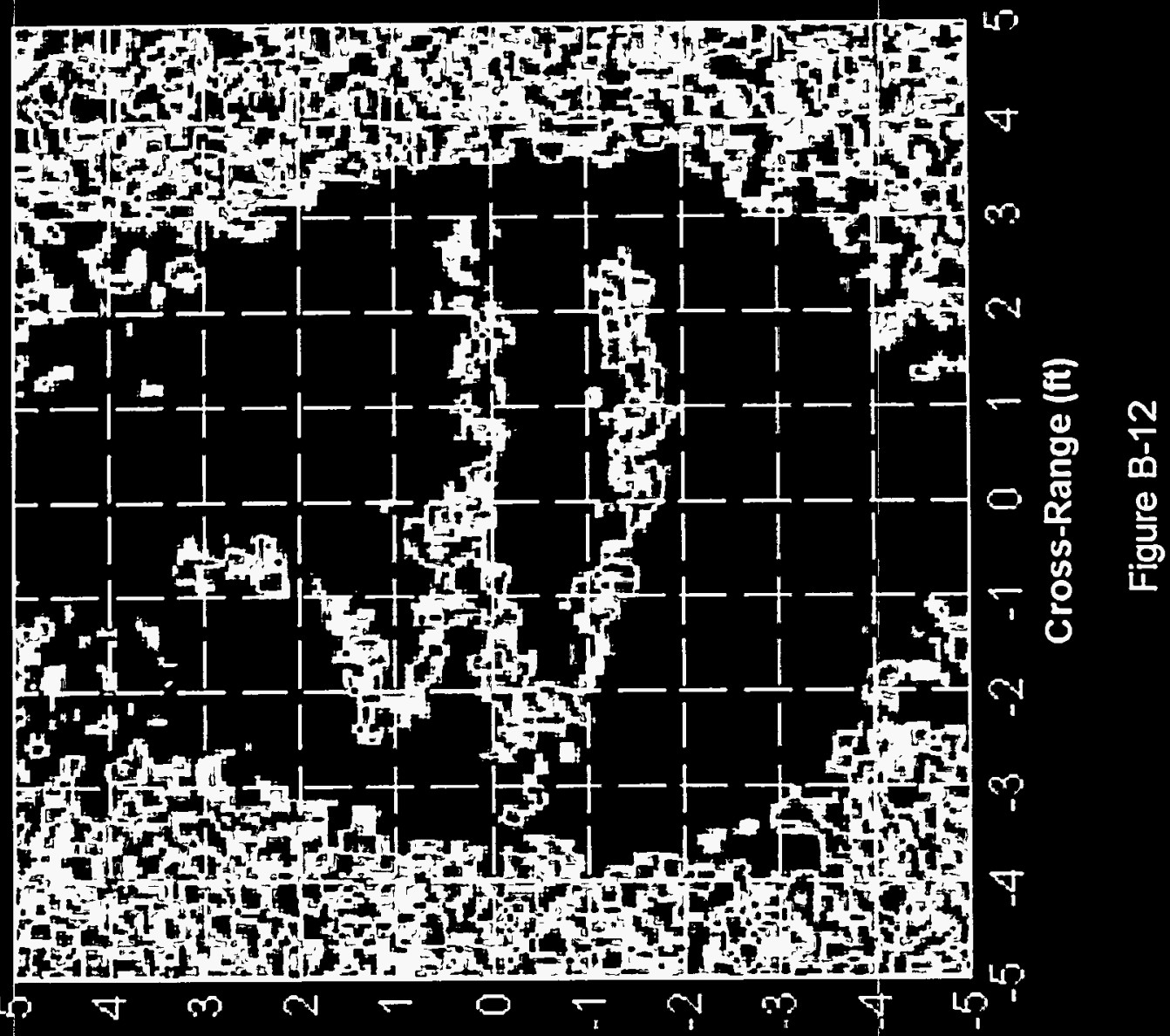

(y) әбиey-uмо

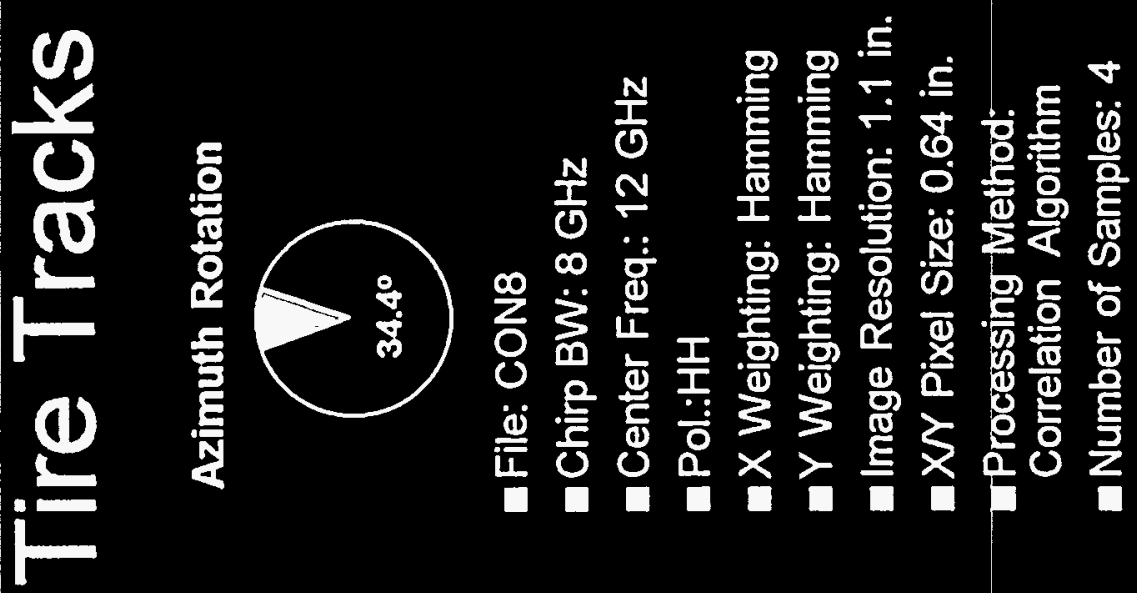




\section{Tire Tracks (Wet)}

\section{Azimuth Rotation}

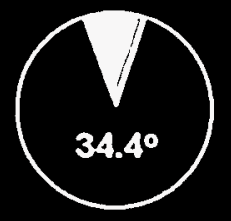

afile: CON9

Chirp BW: $8 \mathrm{GHz}$

Center Freq.: 12 GHz

Pol.:HH

$X$ Weighting: Hamming

$Y$ Weighting: Hamming

Image Resolution: 1.1 in.

aXY Pixel Size: 0.64 in.

Processing Method:

Correlation Algorithm

- Number of Samples: 4
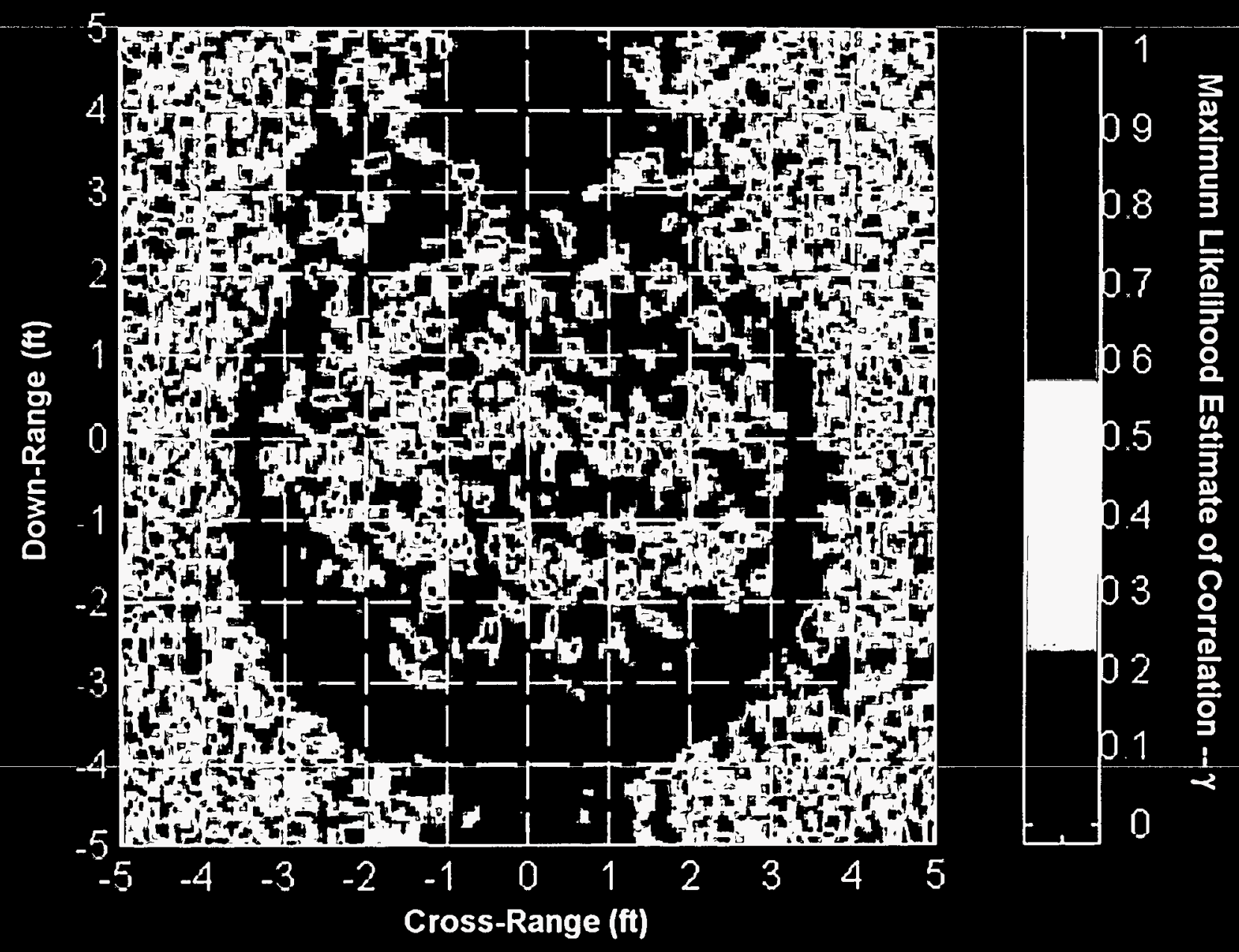

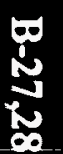

Figure B-13 
Maximum Likelihood Estimate of Correlation $-\gamma$

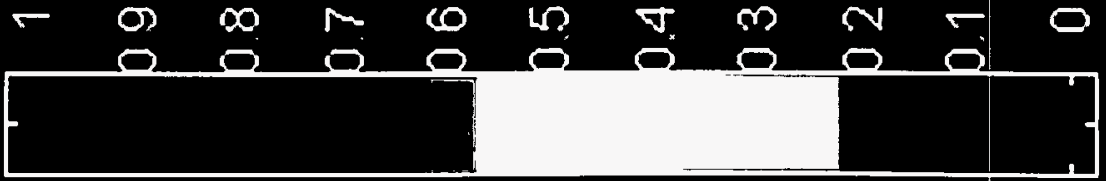

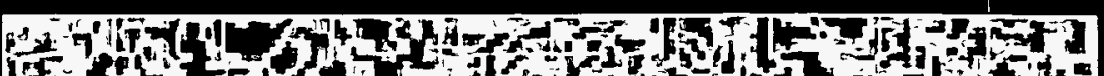
(5)

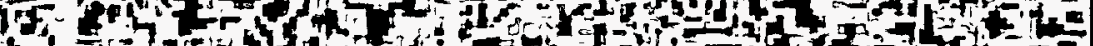

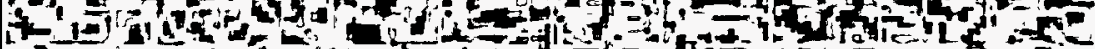

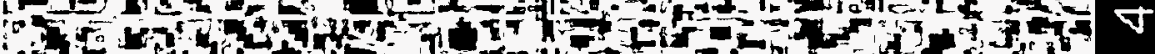

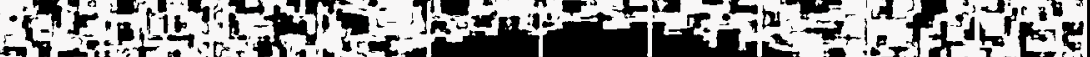

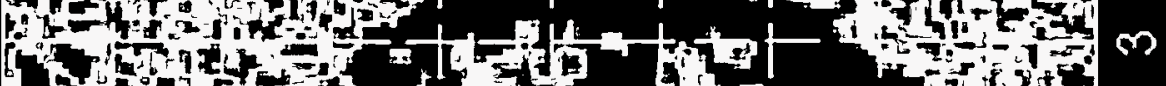

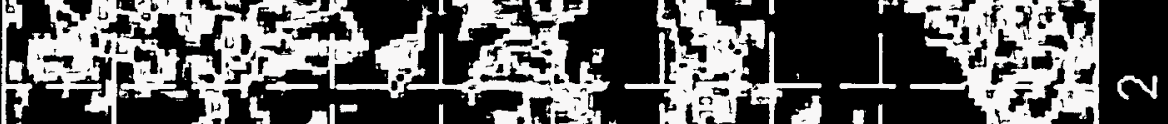

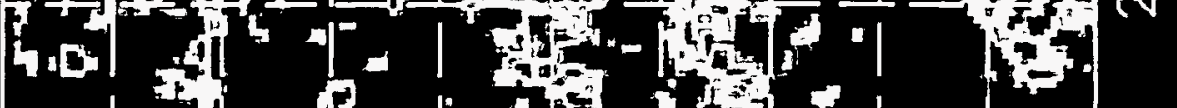

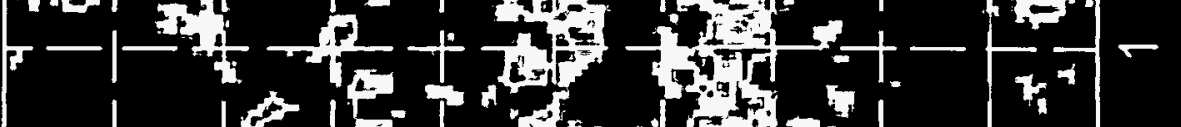

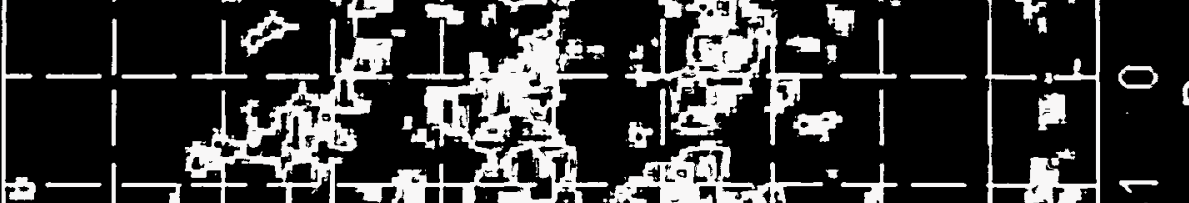

0 a

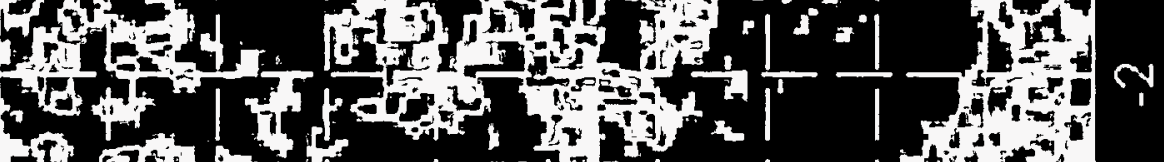

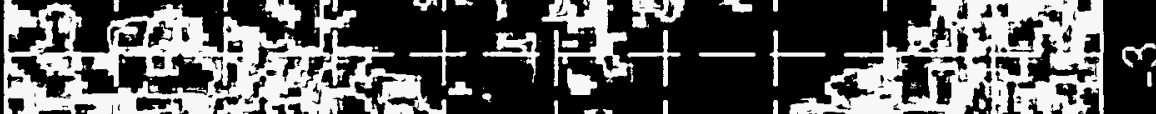

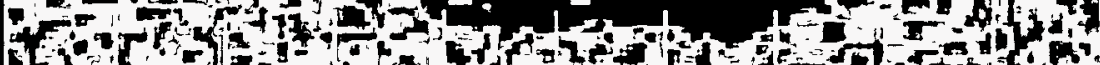
LAt

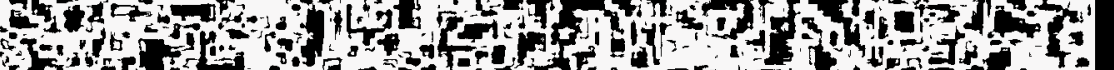
Hering

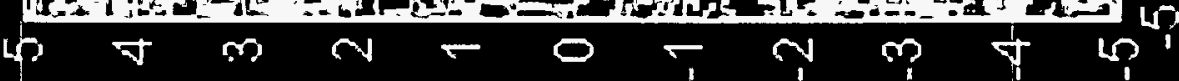

(y) әб̆uey-umog
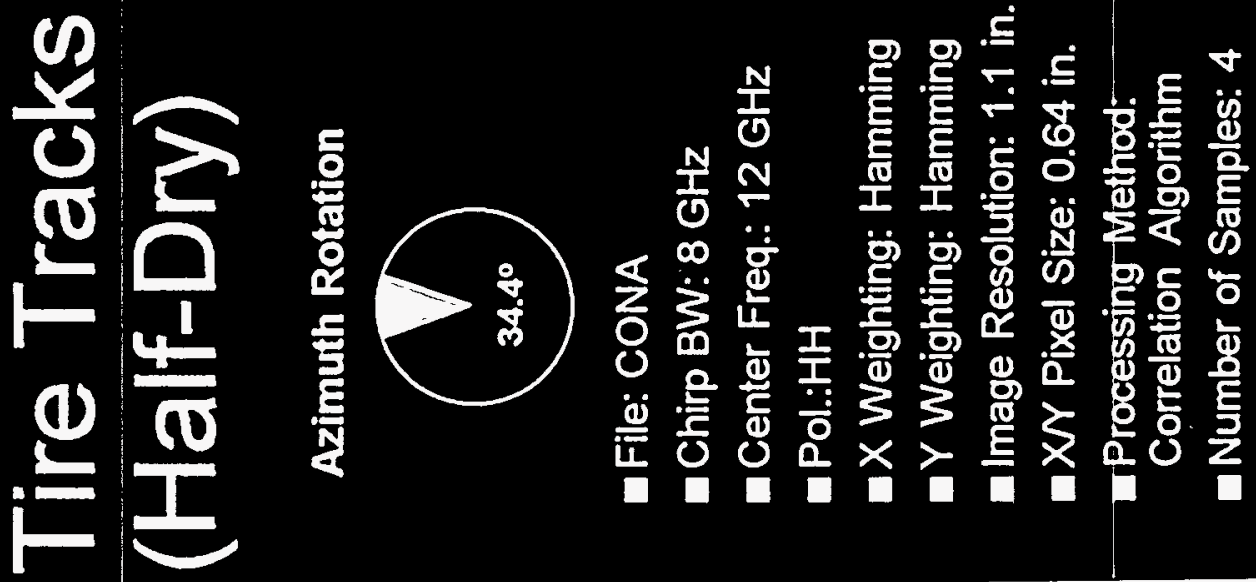
Maximum Likelihood Estimate of Correlation $-\gamma$
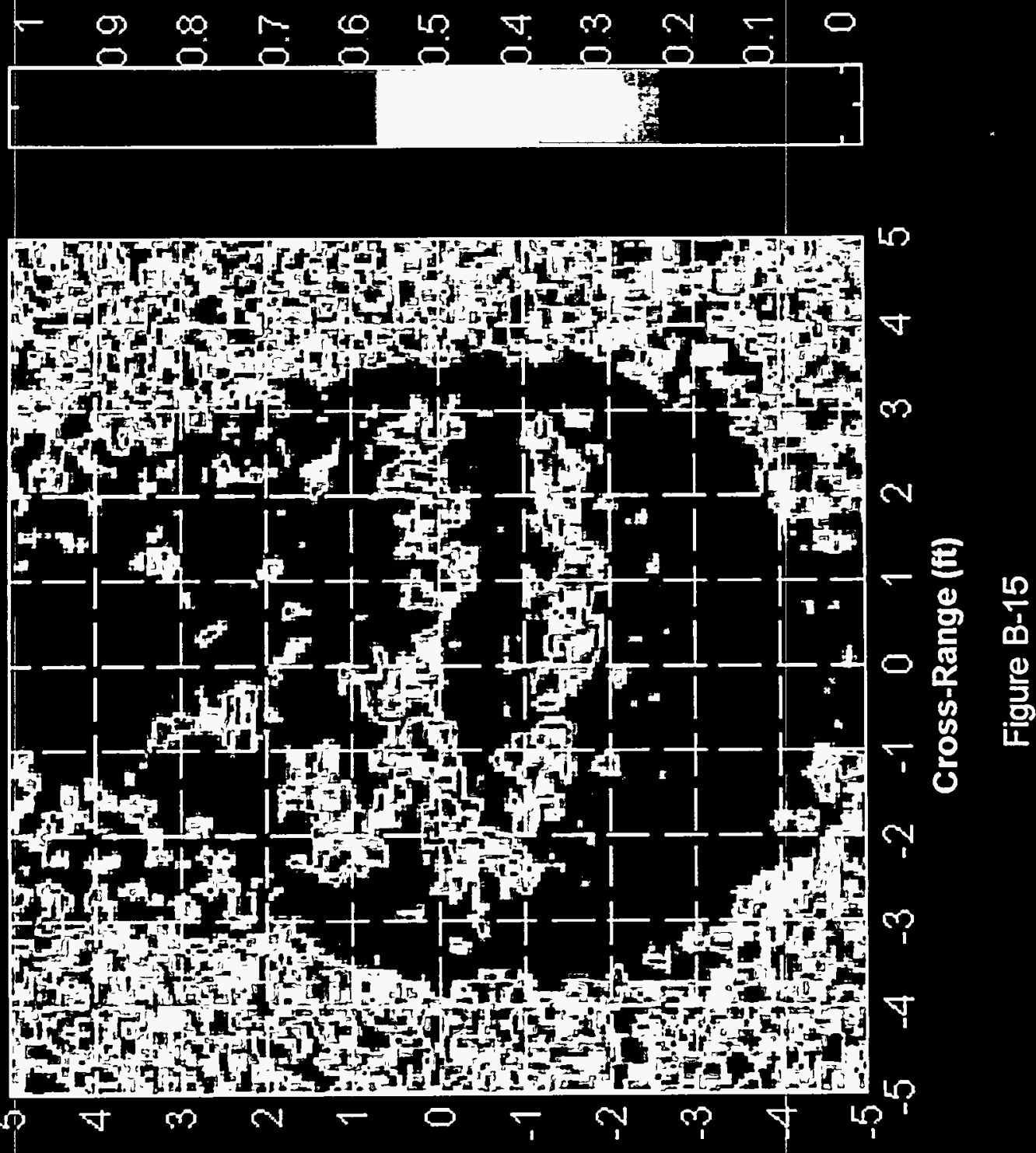

(y) әอินey-umog

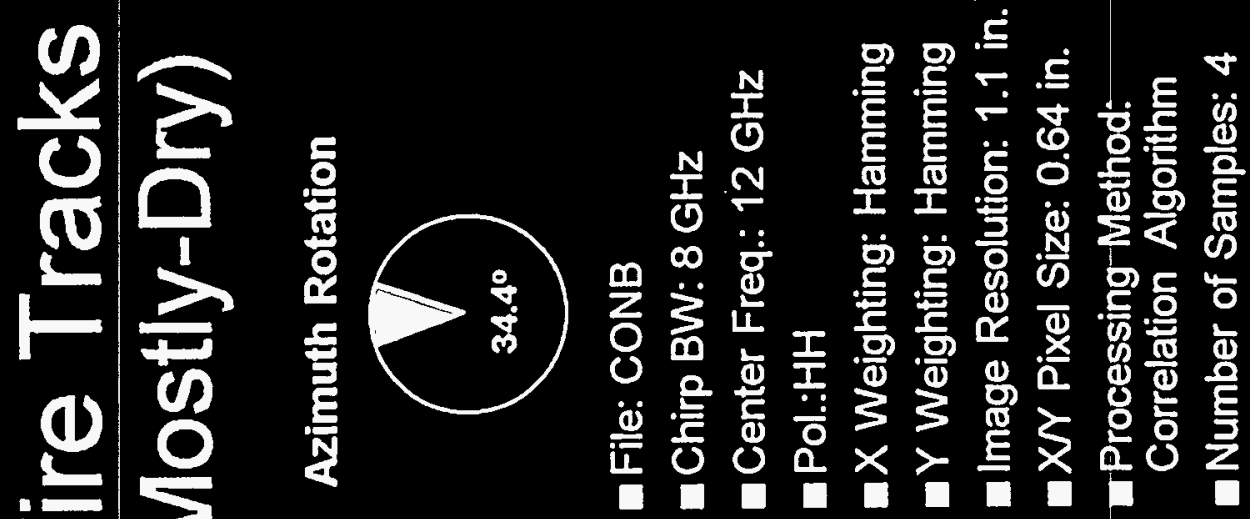




\section{Tire Tracks ("Dry")}

Azimuth Rotation

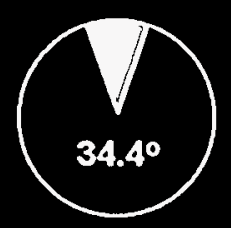

aFile: CONC

Chirp BW: $8 \mathrm{GHz}$

Center Freq.: $12 \mathrm{GHz}$

aPol.:HH

$\triangle X$ Weighting: Hamming

$Y$ Weighting: Hamming

Image Resolution: 1.1 in.

aXY Pixel Size: 0.64 in.

-Processing-Method:

Correlation Algorithm

number of Samples: 4
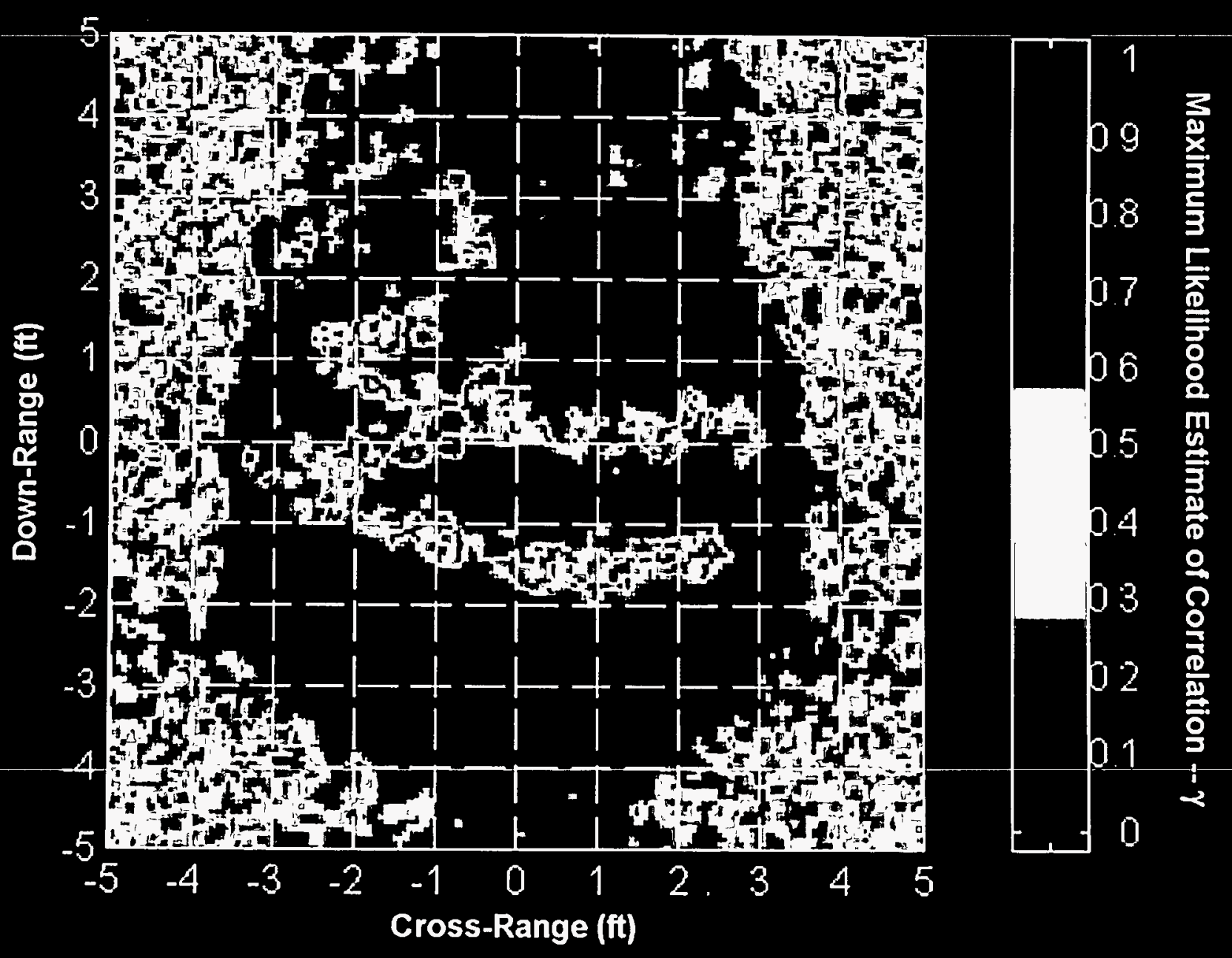

$W$
$\omega$
$\omega$
$\omega$

Figure B-16 
Maximum Likelihood Estimate of Correlation $-\gamma$

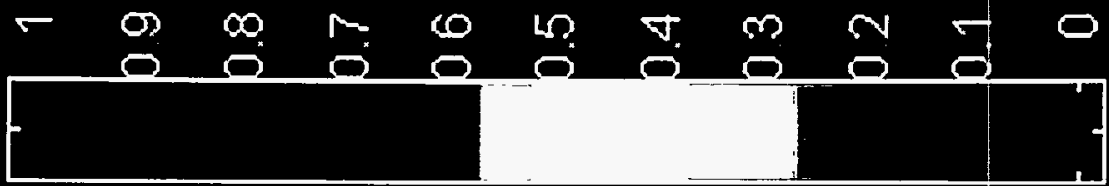

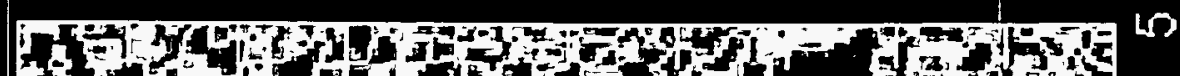

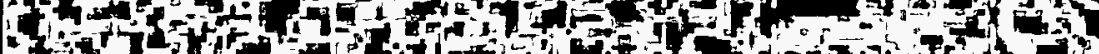

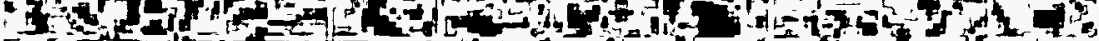

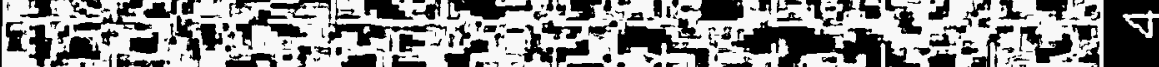

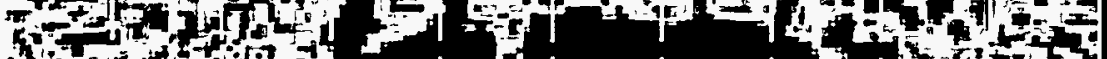

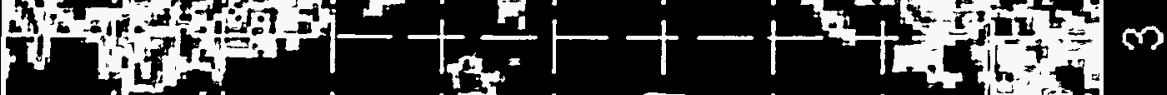

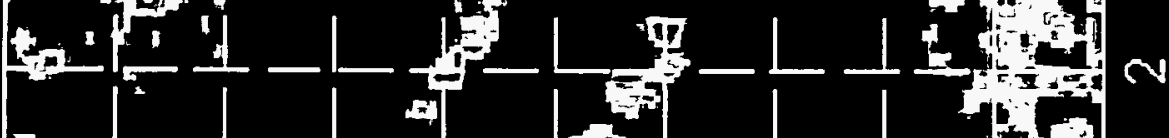

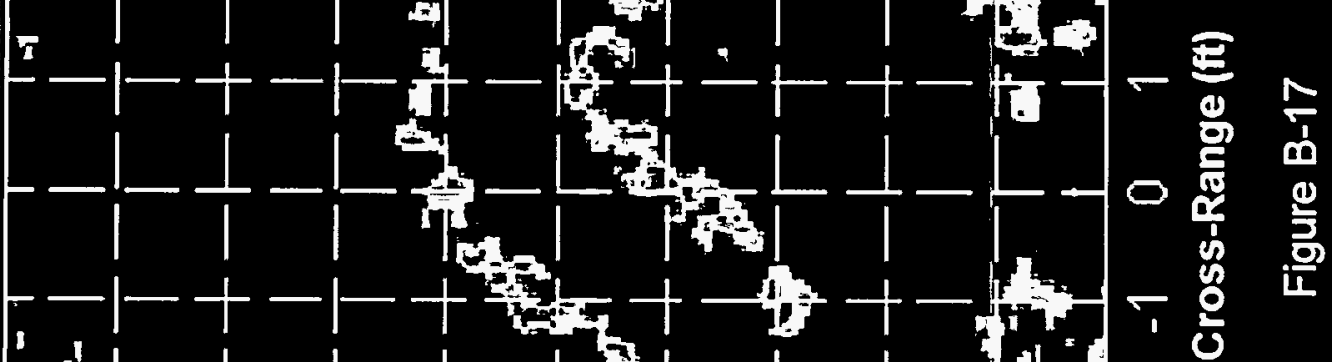

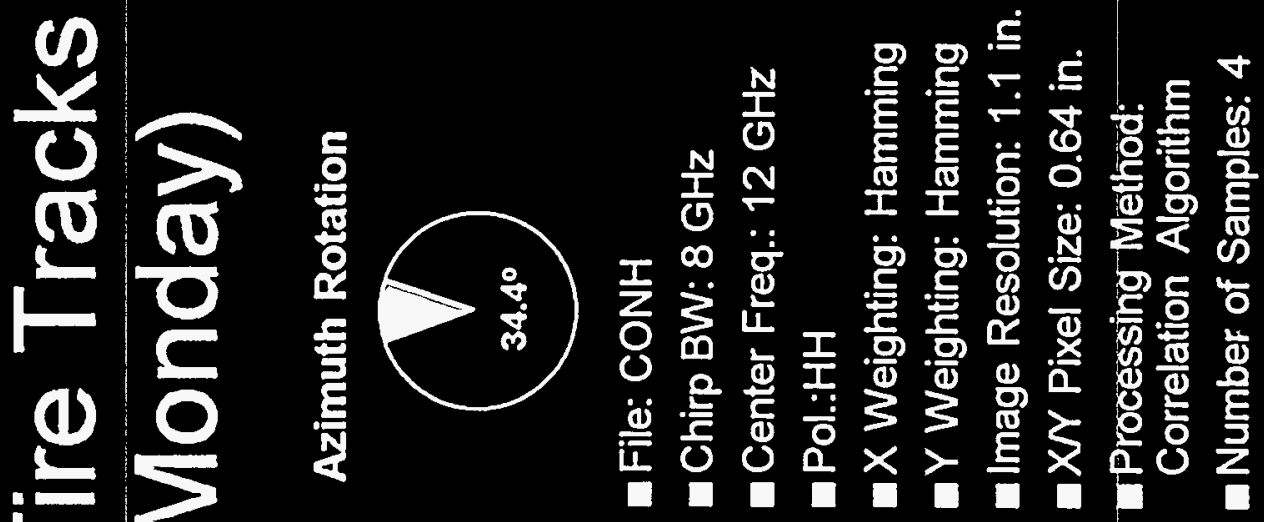




\section{Tire Tracks (Tuesday)}

Azimuth Rotation

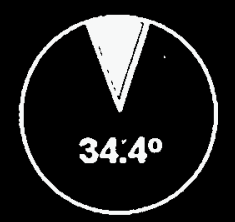

-File: CONI

aChirp BW: $8 \mathrm{GHz}$

LCenter Freq.: $12 \mathrm{GHz}$

a Pol.:HH

X Weighting: Hamming

aY Weighting: Hamming

- Image Resolution: 1.1 in.

aXY Pixel Size: 0.64 in.

Processing Method:

Correlation Algorithm

number of Samples: 4

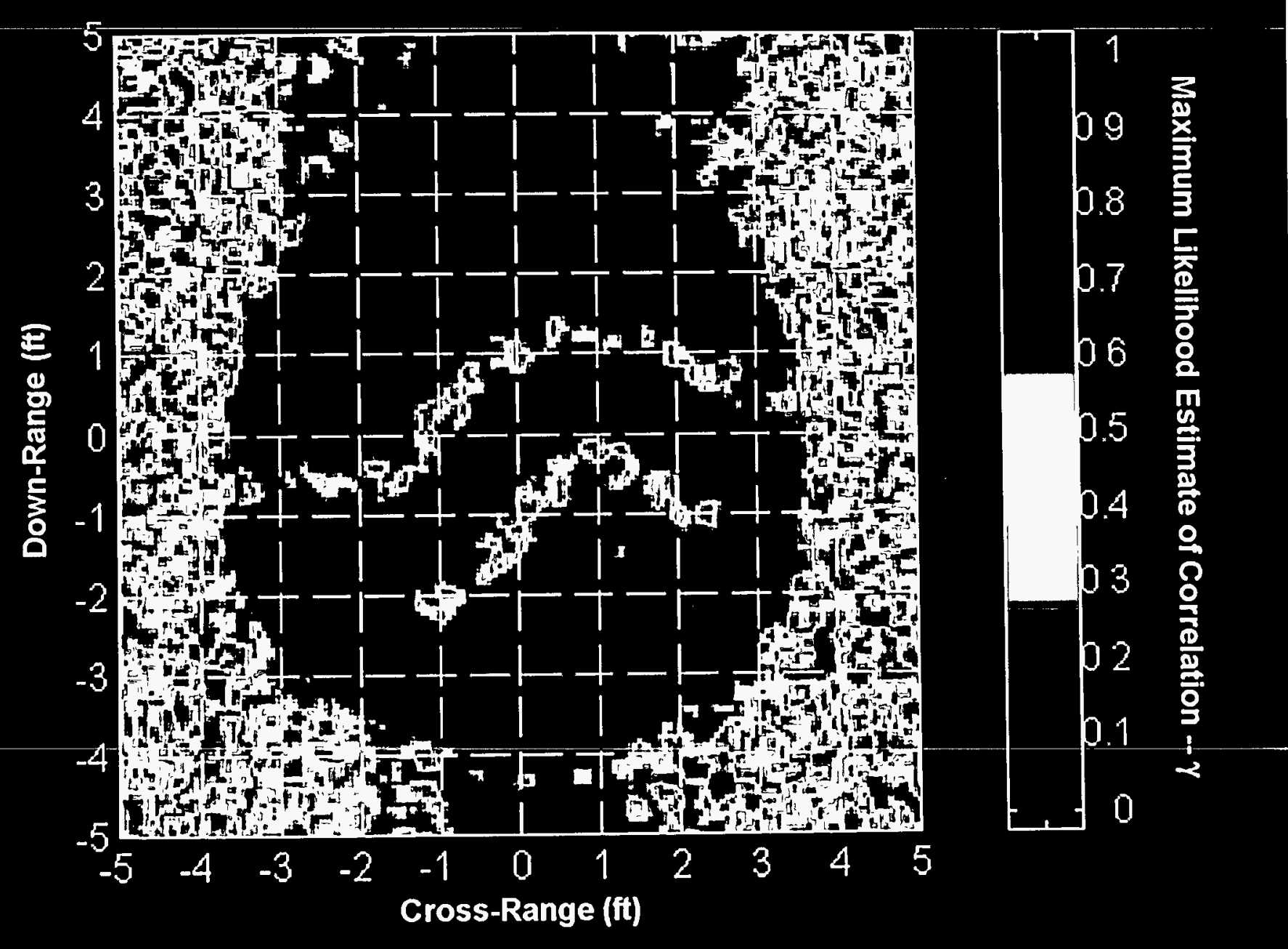

$\underset{\infty}{W}$

Figure B-18 


\section{Tire Tracks (Wednesday)}

\section{Azimuth Rotation}

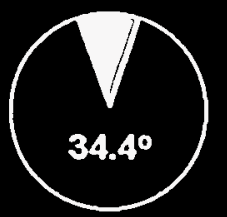

File: CONJ

Chirp BW: $8 \mathrm{GHz}$

Center Freq.: $12 \mathrm{GHz}$

-Pol.:HH

$\times$ Weighting: Hamming

aY Weighting: Hamming

almage Resolution: 1.1 in.

$\times N$ Pixel Size: 0.64 in.

uProcessing Method:

Correlation Algorithm

- Number of Samples: 4
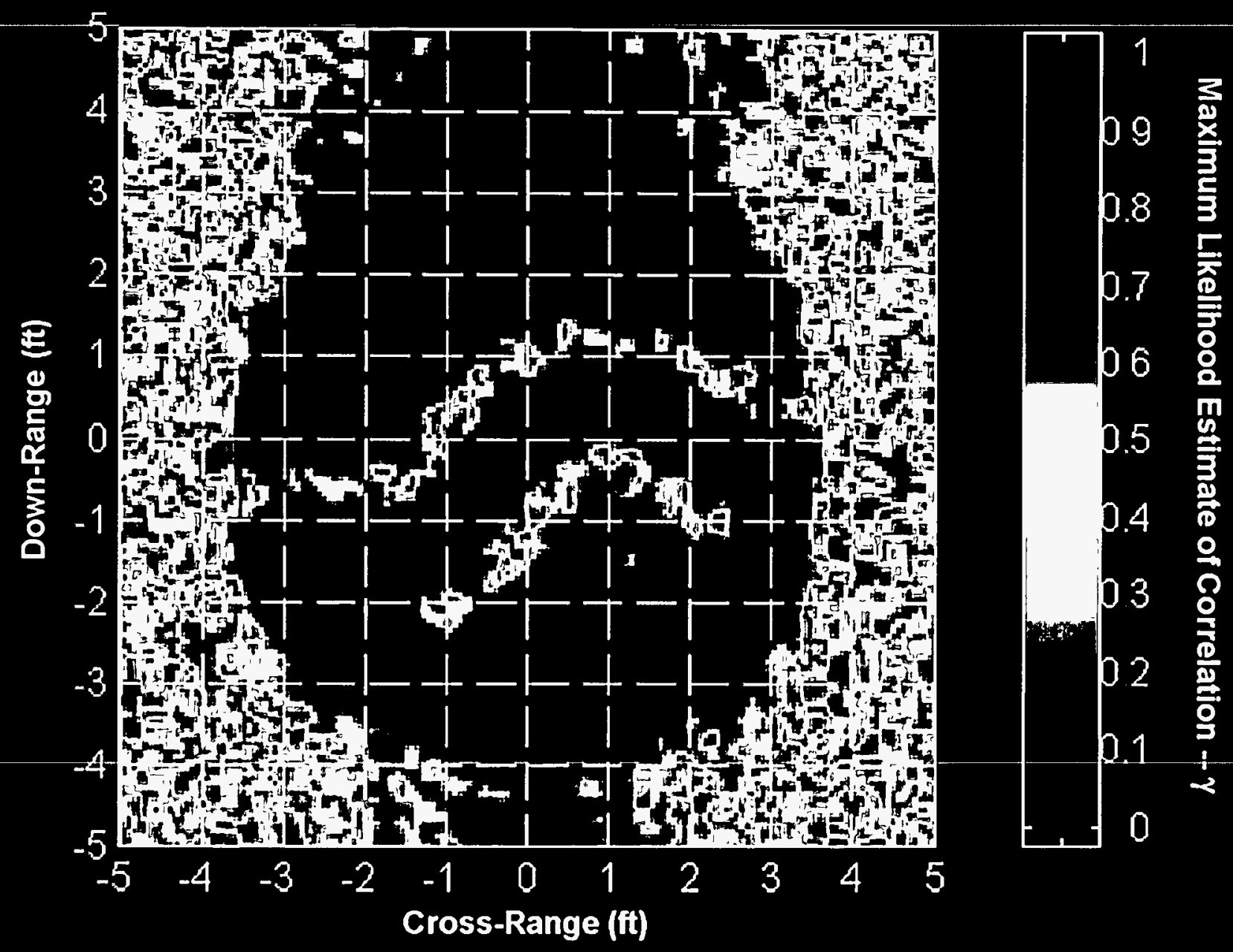

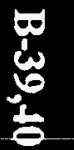

Figure B-19 


\section{Tire Tracks (Thursday)}

Azimuth Rotation

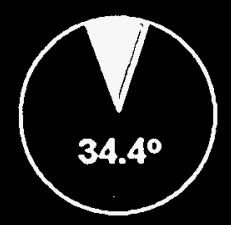

aFile: CONK

Chirp BW: $8 \mathrm{GHz}$

Center Freq.: $12 \mathrm{GHz}$

$\llcorner$ Pol.:HH

$\llcorner X$ Weighting: Hamming

$\llcorner Y$ Weighting: Hamming

- Image Resolution: 1.1 in.

a N Pixel Size: 0.64 in.

-Processing-Method:-

Correlation Algorithm

n Number of Samples: 4

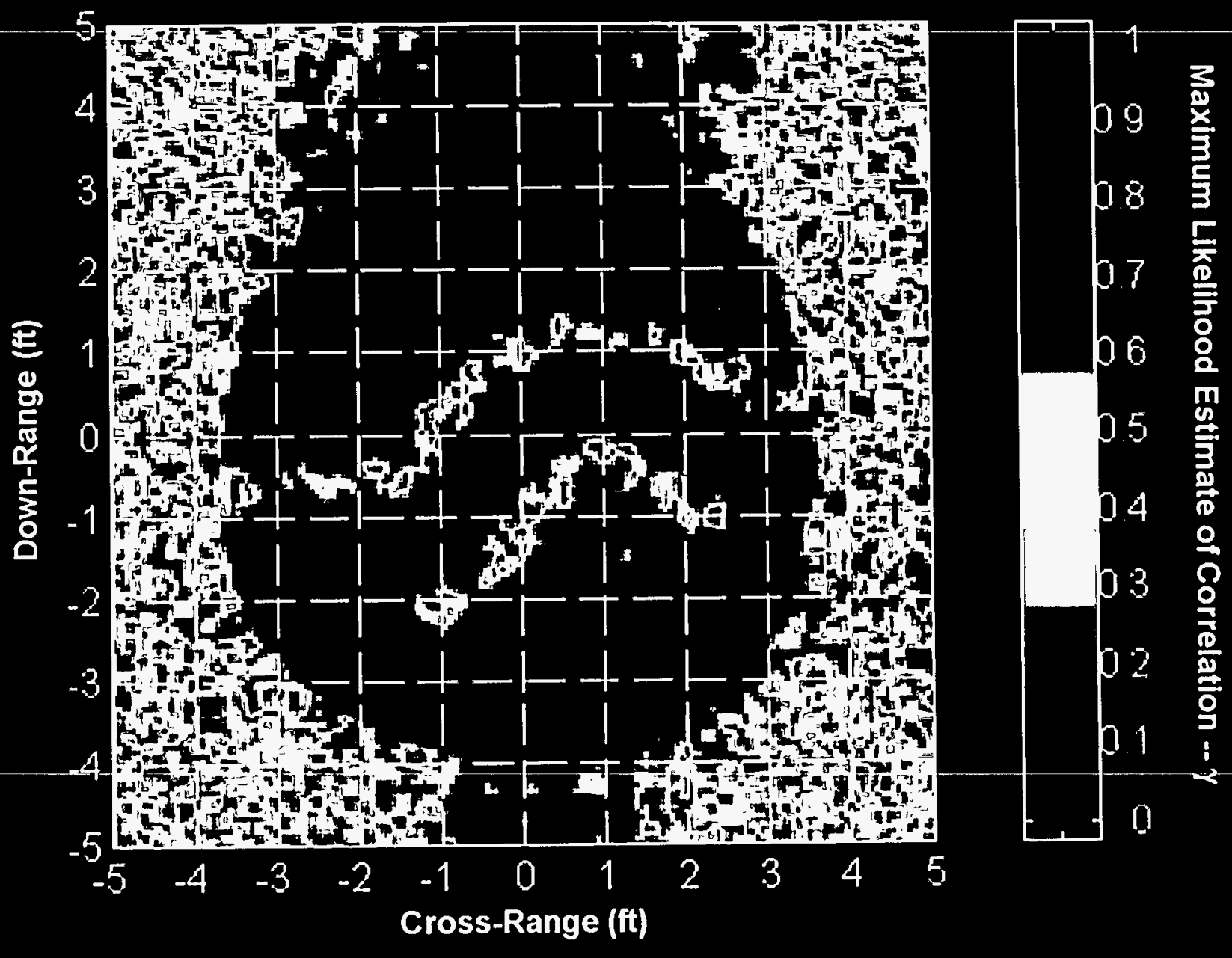

$\underset{5}{5}$

Figure B-20 


\section{Tire Tracks (Friday)}

Azimuth Rotation

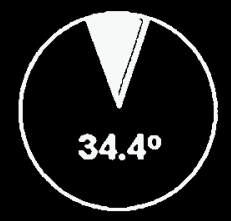

afile: CONL

aChirp BW: $8 \mathrm{GHz}$

( Center Freq.: $12 \mathrm{GHz}$

Dol::HH

$\square X$ Weighting: Hamming I $Y$ Weighting: Hamming almage Resolution: 1.1 in.

$\square \times N$ Pixel Size: 0.64 in.

Processing Method: Correlation Algorithm

n Number of Samples: 4
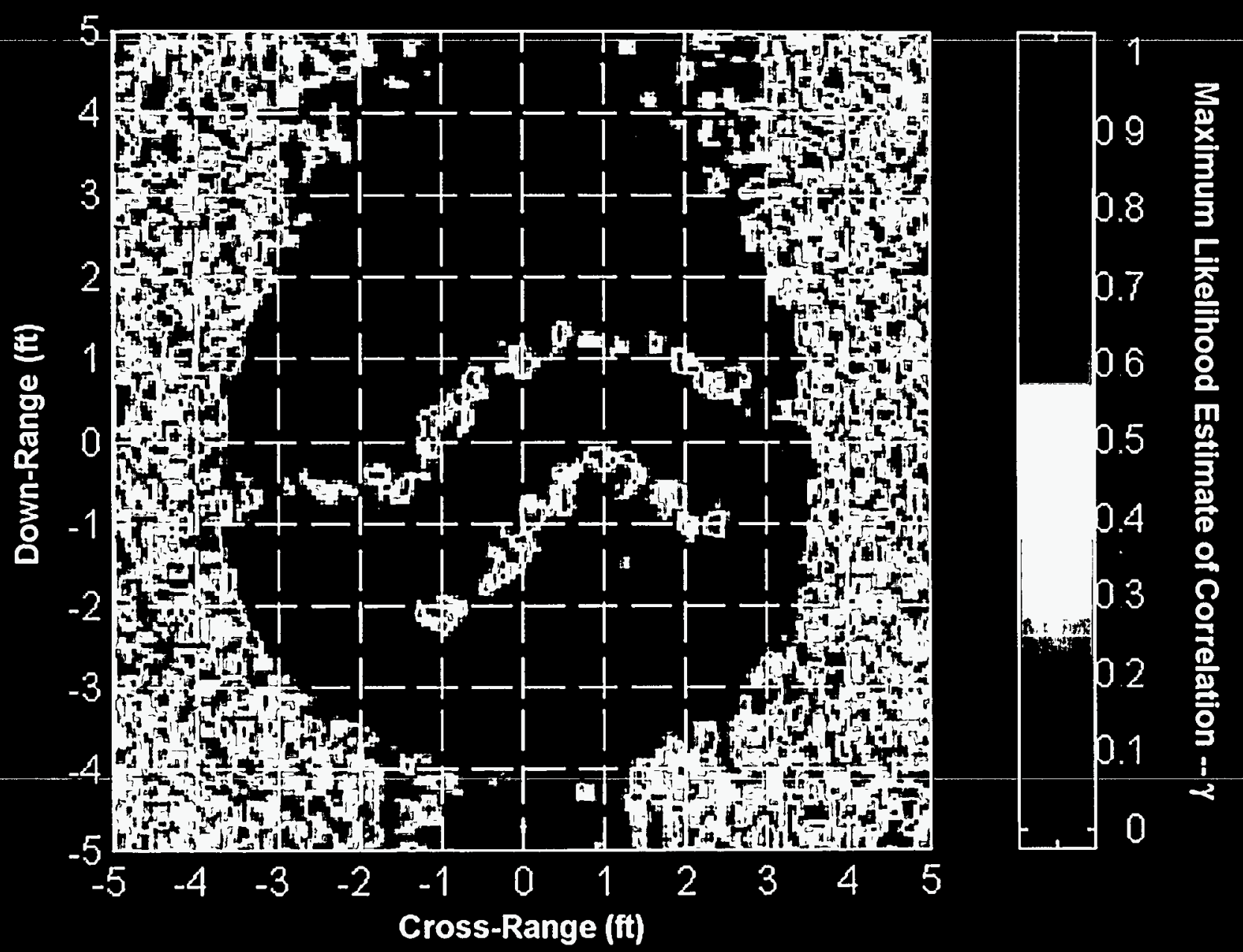

5
5
5
5

Figure B-21 